\title{
The ROSAT all-sky survey catalogue of the nearby stars ${ }^{\star}$
}

\author{
M. Hünsch ${ }^{1}$, J.H.M.M. Schmitt ${ }^{2,4}$, M.F. Sterzik ${ }^{3,4}$, and W. Voges ${ }^{4}$ \\ 1 Institut für Theoretische Physik und Astrophysik, Universität Kiel, Olshausenstrasse 40, 24118 Kiel, Germany \\ 2 Hamburger Sternwarte, Universität Hamburg, Gojenbergsweg 112, 21029 Hamburg, Germany \\ 3 European Southern Observatory, Casilla 19001, Santiago 19, Chile \\ 4 Max-Planck-Institut für extraterrestrische Physik, Giessenbachstr. 1, 85740 Garching, Germany
}

Received October 16, accepted November 16, 1998

\begin{abstract}
We present X-ray data for all entries of the Third Catalogue of Nearby Stars (Gliese \& Jahreiß 1991) that have been detected as X-ray sources in the ROSAT all-sky survey. The catalogue contains 1252 entries yielding an average detection rate of 32.9 percent. In addition to count rates, source detection parameters, hardness ratios, and X-ray fluxes we also list X-ray luminosities derived from Hipparcos parallaxes.
\end{abstract}

Key words: stars: activity — stars: coronae — stars: late-type - X-rays: stars

\section{Introduction}

One of the major results obtained with the imaging X-ray telescopes flown onboard the Einstein and ROSAT observatories is the ubiquity of X-ray emission from normal stars throughout the whole Hertzsprung-Russell (HR) diagram, the only exceptions being A-type main sequence stars and some of the late-type giants and supergiants. However, the more sensitive ROSAT observations have revealed X-ray emission even from those types of stars.

$\mathrm{X}$-ray emission from late-type stars is generally attributed to magnetically heated stellar coronae. As suggested by the example of the Sun's corona, the X-ray emitting hot $\left(>10^{6} \mathrm{~K}\right)$ plasma is believed to be confined by coronal magnetic fields, which ultimately originate from the interaction between rotation and outer convection zones. The solar X-ray luminosity varies approximately between $310^{25}$ and $110^{27} \mathrm{erg} \mathrm{s}^{-1}$ (extrapolated to a $1-300 \AA$ "bolometric X-ray band";

Send offprint requests to: M. Hünsch,

e-mail: huensch@astrophysik.uni-kiel.de

* Catalogue also available at CDS via anonymous ftp to cdsarc.u-strasbg.fr (130.79.128.5) or via http://cdsweb.ustrasbg.fr/Abstract.html cf. Haisch \& Schmitt 1996; Acton 1996) during the solar cycle and is known to be strongly correlated with other magnetic activity indicators (e.g., sunspot numbers, flare frequency, chromospheric Ca II emission). Hence, X-ray luminosity can be regarded as a good activity indicator also for other late-type stars. Conversely, X-ray emission from O- and early B-type stars obeys (approximately) a $L_{\mathrm{x}} / L_{\mathrm{bol}} \approx 10^{-7}$ relation and is attributed to shocks generated and dissipated within the radiatively driven winds of those stars.

A thorough investigation of the properties of stellar $\mathrm{X}$-ray emission requires large samples of stars in order to reduce the effects of individual scatter and to construct samples of stars with given properties such as mass, age, rotation rate etc. Especially important are complete, volume-limited samples of stars, for example, to derive X-ray luminosity distribution functions. In principle, there are two different approaches: i) to construct such a complete sample and to look for X-ray emission from those stars afterwards, and ii) to scan the whole sky for $\mathrm{X}$-ray emission and then to extract the data for the sample stars (input positions). The first method has been applied by Schmitt et al. (1995) and Schmitt (1997) for the immediate solar environment, i.e., the K- and M-type stars within $7 \mathrm{pc}$ and the A-, F-, and G-type stars within $13 \mathrm{pc}$ from the Sun, respectively. These investigations revealed that virtually every late-type dwarf star with spectral type later than A7 can be detected as an X-ray source given data of sufficient sensitivity. These data are from both the ROSAT all-sky survey (RASS) and deep individual pointed ROSAT observations. A similar investigation on late-type giants within $25 \mathrm{pc}$ has been performed by Hünsch et al. (1996).

The second method requires the existence of a sufficiently deep all-sky survey. Until now, the ROSAT observatory has undertaken the only sensitive X-ray survey. These data provide a flux-limited but otherwise unbiased sample of X-ray sources (Voges et al. 1996a). Of the $\approx 1.510^{5}$ detected sources, about one third are 
considered to be coronal. We used these data to search for X-ray emission from stars contained in the Catalogue of Nearby stars (the so-called Gliese catalogue; Gliese \& Jahreiß 1991). The Gliese catalogue is the most comprehensive list of stars in the solar environment and it is proposed to be as complete as possible within a space volume of $25 \mathrm{pc}$ radius around the Sun. Our investigation is limited in completeness by both the completeness of the Gliese catalogue and the sensitivity of the RASS. However, also for those Gliese stars that are not detected, it is in principle possible to estimate upper limits for their X-ray luminosity (see Sect. 2.1).

A preliminary investigation of stellar activity of the nearby stars based on the data presented here has already been performed by Sterzik \& Schmitt (1997). However, $\mathrm{X}$-ray luminosities for those stars derived from ROSAT data combined with Hipparcos parallaxes are presented here for the first time.

We have also performed similar surveys of X-ray emission from the stars contained in the Bright Star catalogue (BSC; Hoffleit \& Warren 1991). These results are presented as catalogues of X-ray data, which have already been published for OB stars (Berghöfer et al. 1996), latetype giants and supergiants (Hünsch et al. 1998a; hereafter HSV98), and late-type main-sequence stars and subgiant stars (Hünsch et al. 1998b).

\section{RASS data and detection of late-type stars}

\subsection{The ROSAT all-sky survey (RASS)}

During its first half year of operations, the ROSAT observatory carried out the first all-sky survey with an imaging X-ray telescope between July 1990 and January 1991. Further survey observations were carried out in February 1991 (2 days) and August 1991 (10 days). The whole sky was scanned along great circles perpendicular to the direction to the Sun. Because of the Earth's motion around the Sun, the plane of these circles slowly $\left(1^{\circ} / \mathrm{d}\right)$ rotated around an axis through the ecliptic poles, thus covering the whole celestial sphere within 6 months. Each point of the sky was observed several times as the scan paths of 2 degrees width (i.e., the field of view of the PSPC detector) progressed along the ecliptic. Therefore, the data of any particular source consist of a number of "snapshots" of up to $30 \mathrm{~s}$ duration, separated by the orbital period of the satellite $(\approx 90 \mathrm{~min})$ and distributed over an interval of at least 2 days. Towards the ecliptic poles, the cumulative exposure time increases due to the larger number of scans covering a particular celestial position. Depending on the ecliptic latitude (and down-time due to passages through the radiation belts of the Earth), the effective exposure time varies between $\sim 100 \mathrm{~s}$ and $\sim 40000 \mathrm{~s}$ (at the poles), with typical values of $\sim 400 \mathrm{~s}$ at the ecliptic. Typical limiting RASS count rates are $\approx 0.015 \operatorname{cts~s}^{-1}$; given a typical energy-conversion factor for soft sources of $610^{-12} \mathrm{erg} \mathrm{cts}^{-1} \mathrm{~cm}^{-2}$ (cf. Sect. 2.3) the typical detection limit of RASS observations amounts to a limiting flux of $f_{\mathrm{x}} \approx 10^{-13} \mathrm{erg} \mathrm{cm}^{-2} \mathrm{~s}^{-1}$. At the distance limit of the Gliese catalogue, i.e., at $25 \mathrm{pc}$, this corresponds to an X-ray luminosity of $L_{\mathrm{x}} \approx 7.510^{27} \mathrm{erg} \mathrm{s}^{-1}$. Note that this is not an average value for the detection limit for the Gliese stars since many stars are closer and the exposure time increases towards the ecliptic poles.

For a more detailed description of the RASS we refer to Voges (1992) and Belloni et al. (1994). Details of the ROSAT observatory in general can be found in Trümper (1983) and Trümper et al. (1991). The PSPC detector used during the RASS is described by Pfeffermann et al. (1986). In February 1997 the remaining gaps left in the allsky survey were filled with a sequence of more than 500 pointed, partially overlapping PSPC observations so that with the exception of a small region around the strong X-ray source Sco X-1 the whole sky has been imaged with the ROSAT PSPC. In the catalogue presented in this paper we include sources detected in this "survey repair" pointed observations; they are marked with an asterisk.

The source detection was performed by means of a maximum likelihood algorithm (Cruddace et al. 1988) in the course of the standard analysis software system (SASS; Voges et al. 1992). The significance of an X-ray source is expressed by the likelihood $\mathrm{Li}=-\ln (1-P)$, where $P$ is the probability of existence; e.g., a likelihood of $\mathrm{Li}=7$ corresponds to a source existence probability of $99.9 \%$. The result of the SASS is a comprehensive list of approximately $10^{5}$ sources, each described by the sky position in right ascension and declination, its source detection likelihood, count rate, hardness ratios, extent, and corresponding errors. The data for the brighter X-ray sources have been released as the ROSAT All-sky Survey Bright Source Catalogue (Voges et al. 1996b), which contains sources with Likelihood $\geq 15$, count rate larger than $0.05 \mathrm{~s}^{-1}$, and with at least 15 detected photons.

\subsection{Identification of X-ray sources with nearby stars}

We used the Third Catalogue of Nearby Stars (Gliese \& Jahreiß 1991) as input sample for our search of $\mathrm{X}$-ray bright nearby stars. That input sample consists of 3802 stars.

The procedure whereby the positions of RASS sources were matched with the stars of our input sample is the same as described in HSV98. We accepted sources with a likelihood greater than or equal to 7 within 90 arcsec distance from the input stars. As for the BSC sample, the choice of this cut-off radius is empirically justified by means of a Monte Carlo simulation of about the same number of random positions as input positions. However, since the binary fraction is much larger in the Gliese catalogue than in the BSC, the number of independent input 
positions is significantly smaller than the total number of catalogue entries; we therefore combined all binaries into one input position for each system, since most of them are too close to be separated with the RASS data. This results in only 3365 independent input positions, for which we determined the distribution of offsets. The same number of random positions results in 112 (artificial) matches with X-ray sources, yielding a mean of $1.110^{-3}$ matches per square arcsec in the offset distribution plane, i.e., about one third of that of the random sample used for the 9110 BSC stars (as expected). At 90 arcsec offset the number of matches of X-ray sources with real stars exceeds the number of artificial matches by a factor of 2 . That means, at 90 arcsec offset between optical and X-ray position the differential probability that the X-ray source can be attributed to the star (and not to a background object) is $50 \%$. This differential probability increases very rapidly for smaller values of positional offset, while for even larger values of offset the chance for obtaining a spurious identification exceeds that of finding the true X-ray counter part.

We note that the accuracy of the input positions in the Gliese catalogue (given only to integers of seconds in RA and tenth of arcminutes in Dec) is less than for the BSC stars, hence resulting in a somewhat broader distribution of the offsets for the real stars. On the other hand, the intrinsic detection probability is larger for the Gliese stars than for the BSC stars because the Gliese stars are closer to us and the content of late-type stars is much larger. This would cause a somewhat steeper distribution of the offsets. Probably, both effects compensate each other, thus leading to a $50 \%$ differential probability for a correct identification at essentially the same offset value.

Of the X-ray sources extracted by the match procedure, 469 are rather weak sources that are not included in the Bright Source Catalogue (Voges et al. 1996b). We checked their X-ray images by eye for reality. Specifically, we rejected photon distributions that are significantly contaminated by nearby strong sources or that are obviously extended. In questionable cases, we ran the standard source detection algorithm of EXSAS on the source images in different passbands and decided on the basis of the results which sources to retain in our final catalogue.

Confining now attention to the 3365 (independent) input positions identified with Gliese stars, we detected X-ray emission from 1252 stars, i.e., the average detection rate is $37 \%$. Since the total search area around these 3365 stars is $3365 \cdot \pi \cdot\left(1.5^{\prime}\right)^{2}=6.61 \square^{\circ}=0.016 \%$ of the sphere, and the total number of RASS sources amounts to $\sim 150000$, we would expect 24.0 chance coincidences of Gliese stars with background (or foreground) X-ray sources (i.e., $1.92 \%$ of our detected sources).

\subsection{Determination of X-ray fluxes and luminosities}

The procedure of determining X-ray fluxes has also been described in HSV98. In this paper, we followed the same procedure, except using a slightly different formula for the calculation of individual energy-conversion factors

$\mathrm{ECF}=(5.30 \cdot H R+8.31) 10^{-12} \mathrm{erg} \mathrm{cm}^{-2} \mathrm{cts}^{-1}$

which was derived by Schmitt et al. (1995) from an X-ray study of a complete sample of main-sequence stars within $7 \mathrm{pc}$ distance; here $H R$ denotes the hardness ratio defined through

$H R=\frac{H-S}{H+S}$,

where $H$ and $S$ denote the source counts in the hard (0.5$2.0 \mathrm{keV})$ and soft $(0.1-0.4 \mathrm{keV})$ passbands of ROSAT. The hardness ratio is an "X-ray color" that is influenced by both the plasma temperature and the hydrogen column density.

Since the SASS source detection was separately performed in both passbands and since most of our X-ray sources were detected in both bands, the hardness ratios can be estimated for many stars, although in some cases with quite substantial errors. In a few cases, when the sources were not detected in either the soft or the hard passband, we set $H R=+1.0$ or -1.0 by definition, respectively. We refrain from estimating individual errors for $f_{\mathrm{x}}$ since the error in ECF is very difficult to quantify. In general, we estimate this error to be within a factor of two for the weaker sources and less for the brighter sources.

The X-ray luminosities are calculated by the relation

$L_{\mathrm{x}}=4 \pi d^{2} \times f_{\mathrm{x}}$

where $d$ is the distance to the star. We used the distances revised on the basis of the Hipparcos parallaxes (ESA 1997) and kindly made available to us by H. Jahreiß. No X-ray luminosities are computed for those few stars for which no reliable distances exist. Note that the catalogue contains a few stars which obviously do not belong to the solar environment but were erroneously included in the third version of the Gliese catalogue.

\section{The catalogue}

The table contains optical and X-ray data of all 1252 detected Gliese stars. Note that many entries are actually binaries. In a few cases, both components have been detected separately. However, in most cases, the detected $\mathrm{X}$-ray source is attributed to both components and no attempt is made to split up the X-ray flux. As a consequence, care must be taken when interpretating the catalogue information for binaries. For example, in the case of Gl 244 A, B (= Sirius), the X-ray flux is known to arise exclusively from the hot white dwarf B-component, while in the 
case of Gl 280 A, B (= Procyon) the X-ray emission comes from the late-type primary component. In our catalogue, however, the same X-ray flux is attributed to the A- as well as the B-components.

Also note that the quoted count rates are mean count rates during the survey observations, computed as the ratio of total number of counts and integrated exposure time. In some cases, these numbers can strongly be affected by individual flare events (e.g., Gl $877=$ EV Lac, see Schmitt 1994; see also Haisch \& Schmitt 1994).

The columns of the table contain the following information:

Column 1: Designation of catalogue entry. Where available, we prefer the original Gliese (Gl), Gliese-Jahreiß (GJ), or Woolley (Wo) designation. Stars without such a number are named according to one of the following other catalogues (in hierarchical order): Henry Draper (HD), Giclas (G), Luyten Half Second (LHS), Durchmusterung (BD or CD), or various other designations (LTT, LP, GR, Steph, Rob, BPM, AC). Only in very few cases, where we did not find any common designation, do we use the approximate celestial position instead. Stars marked with an asterisk were detected in the "survey repair" observations (cf. Sect. 2.1).

Column 2: $V$ magnitude (from Gliese catalogue).

Column 3: $B-V$ color index (from Gliese catalogue).

Column 4: MK spectral classification (from Gliese catalogue).

Column 5: Distance to the star as given by the Hipparcos parallaxes.

Column 6: effective exposure time in seconds.

Column \%: mean PSPC count rate in counts per second.

Column 8: error of PSPC count rate.

Column 9: likelihood of existence (cf. Sect. 2.1); a value of 999 indicates a source existence likelihood of 999 or greater.

Column 10: offset in arcsec between optical and X-ray position.

Column 11: hardness ratio $H R=(H-S) /(H+S)$ (cf. Sect. 2.3).

Column 12: error of hardness ratio.

Column 13: apparent X-ray flux $(0.1-2.4 \mathrm{keV})$ in $10^{-14} \mathrm{erg} \mathrm{cm}^{-2} \mathrm{~s}^{-1}$ (see Sect. 2.3).

Column 14: X-ray luminosity derived from the distance as given in Col. 5. The values are given in units of $10^{27} \mathrm{erg} \mathrm{s}^{-1}$.

Acknowledgements. We especially thank H. Jahreiß for providing us with an electronic version of the Gliese catalogue and the newest parallaxes of the X-ray detected Gliese stars. The ROSAT project is supported by the Bundesministerium für Bildung, Forschung und Technologie (BMBF/DLR) and the Max-Planck-Gesellschaft (MPG). We would like to thank our collegues from the MPE ROSAT group for their support.

\section{References}

Acton L.W., 1996, in: Pallavicini R., Dupree A.K. (eds.) Cool Stars, Stellar Systems, and the Sun, Proc. 9th Cambridge Workshop, ASP Conf. Ser. 109, 45

Belloni T., Hasinger G., Izzo C., 1994, A\&A 283, 1037

Berghöfer T.W., Schmitt J.H.M.M., Cassinelli J.P., 1996, A\&AS 118, 481

Cruddace R.G., Hasinger G.R., Schmitt J.H.M.M., 1988, The application of a maximum likelihood analysis to detection of sources in the ROSAT data, in: Murtagh F., Heck A. (eds.) Astronomy from Large Databases, ESO Conf. and Workshop Proc. 28, p. 177

ESA 1992, The Hipparcos and Tycho catalogues, ESA SP-1200

Gliese W., Jahreiß H., 1991, Preliminary version of the Third Catalogue of Nearby Stars, on: Brotzmann L.E., Gesser S.E. (eds.) The Astronomical Data Center CD-ROM: Selected Astronomical Catalogues, Vol. 1; NASA/Astronomical Data Center, Goddard Space Flight Center, Greenbelt, MD

Haisch B.M., Schmitt J.H.M.M., 1994, ApJ 426, 716

Haisch B.M., Schmitt J.H.M.M., 1996, PASP 108, 113

Hoffleit D.E., Warren W.H.jr., 1991, The Bright Star Catalogue, 5th rev. ed., Yale Univ. Obs., New Haven

Hünsch M., Schmitt J.H.M.M., Schröder K.-P., Reimers D., 1996, A\&A 310, 801

Hünsch M., Schmitt J.H.M.M., Voges W., 1998a, A\&AS 127, 251 (HSV98)

Hünsch M., Schmitt J.H.M.M., Voges W., 1998b, A\&AS 132, 155

Pfeffermann E., Briel U.G., Hippmann U., et al., 1986, Proc. SPIE 733, 519

Schmitt J.H.M.M., 1994, ApJS 90, 735

Schmitt J.H.M.M., 1997, A\&A 318, 215

Schmitt J.H.M.M., Fleming T.A., Giampapa M.S., 1995, ApJ 450, 392

Sterzik M.F., Schmitt J.H.M.M., 1997, AJ 114, 1673

Trümper J., 1983, Adv. Space Res. 2, No. 4, 241

Trümper J., et al., 1991, Nat 349, 579

Voges W., 1992, in: Proc. of the ISY Conf. "Space Science", ESA ISY-3, ESA Publ., p. 9

Voges W., et al., 1992, in: Proc. of the ISY Conf. "Space Science", ESA ISY-3, ESA Publ., p. 223

Voges W., et al., 1996a, MPE Report 263, 637

Voges W., et al., 1996b, IAUC 6420 


\begin{tabular}{|c|c|c|c|c|c|c|c|c|c|c|c|c|c|}
\hline Name & $V$ & $B-V$ & Sp. type & $d(\mathrm{pc})$ & Exp. & $C R$ & $\pm C R$ & $\mathrm{Li}$ & $\Delta$ & $h r$ & $\Delta h r$ & $f_{\mathrm{x}-14}$ & $L_{\mathrm{x} 27}$ \\
\hline HD 225239 & 6.11 & 0.62 & $\mathrm{G} 2 \mathrm{~V}$ & 36.8 & 262 & 0.628 & 0.052 & 489 & 1 & -0.27 & 0.08 & 432.3 & 699.2 \\
\hline Gl 1 & 8.54 & 1.46 & M4 V & 4.4 & 156 & 0.059 & 0.024 & 9 & 26 & -0.68 & 0.29 & 27.9 & 0.6 \\
\hline Gl $4 \mathrm{~A}$ & 8.97 & 1.44 & $\mathrm{dK} 6 \mathrm{e}$ & 11.8 & 380 & 0.049 & 0.014 & 24 & 7 & -0.42 & 0.25 & 30.1 & 5.0 \\
\hline Gl 4 B & 9.02 & 1.45 & M0.5 V & 11.8 & 380 & 0.049 & 0.014 & 24 & 6 & -0.42 & 0.25 & 30.1 & 5.0 \\
\hline Gl $4.1 \mathrm{~A}$ & 6.43 & 0.64 & G5 V & 20.3 & 508 & 0.323 & 0.026 & 409 & 4 & -0.33 & 0.08 & 212.0 & 104.4 \\
\hline Gl 4.1B & 7.20 & 0.78 & dG8 & 20.3 & 508 & 0.323 & 0.026 & 409 & 4 & -0.33 & 0.08 & 212.0 & 104.4 \\
\hline Gl 5 & 6.14 & 0.75 & K0 Ve & 13.7 & 327 & 0.687 & 0.048 & 624 & 4 & -0.22 & 0.07 & 490.6 & 110.2 \\
\hline G131-026 & 13.54 & & $\mathrm{~m}$ & 10.3 & 251 & 0.090 & 0.021 & 38 & 5 & -0.28 & 0.21 & 61.4 & 7.8 \\
\hline Gl 8 & 2.27 & 0.34 & F2 III-IV & 16.7 & 496 & 0.162 & 0.020 & 153 & 6 & -0.55 & 0.10 & 87.7 & 29.2 \\
\hline G243-016 & 9.48 & 1.22 & $\mathrm{~K} 7$ & 28.0 & 467 & 0.026 & 0.009 & 14 & 25 & -0.54 & 0.30 & 14.1 & 13.2 \\
\hline G242-048 & 11.12 & 1.50 & M1.5 & 19.6 & 836 & 0.028 & 0.007 & 23 & 12 & -0.25 & 0.26 & 19.5 & 8.9 \\
\hline G242-048 & 17.40 & & $\mathrm{~m}$ & 19.6 & 836 & 0.028 & 0.007 & 23 & 15 & -0.25 & 0.26 & 19.5 & 8.9 \\
\hline GR 4 & 14.31 & 1.57 & dM3.4 & & 120 & 0.037 & 0.020 & 8 & 4 & -0.98 & 1.05 & 11.6 & \\
\hline GJ $1006 \mathrm{~A}$ & 12.27 & 1.50 & M4 & 15.1 & 342 & 0.350 & 0.033 & 287 & 10 & -0.28 & 0.09 & 239.2 & 65.5 \\
\hline GJ 1006 B & 13.22 & 1.60 & M4.5 & 15.1 & 342 & 0.350 & 0.033 & 287 & 23 & -0.28 & 0.09 & 239.2 & 65.5 \\
\hline HD 1237 & 6.60 & & G6 V & 17.6 & 231 & 0.388 & 0.045 & 194 & 5 & -0.21 & 0.11 & 279.5 & 103.6 \\
\hline G131-047 & 13.77 & & $\mathrm{~m}$ & 20.8 & 373 & 0.047 & 0.013 & 27 & 12 & -0.27 & 0.26 & 32.4 & 16.8 \\
\hline Gl 14 & 9.00 & 1.36 & dM0.5 & 15.0 & 336 & 0.052 & 0.014 & 30 & 11 & -0.45 & 0.23 & 30.9 & 8.3 \\
\hline G158-052 & 11.00 & 1.42 & dM0 & 35.2 & 356 & 0.034 & 0.012 & 13 & 25 & -0.83 & 0.23 & 13.1 & 19.5 \\
\hline Gl $15 \mathrm{~A}$ & 8.08 & 1.56 & M2 V & 3.6 & 453 & 0.260 & 0.026 & 202 & 18 & -0.66 & 0.08 & 125.2 & 1.9 \\
\hline Gl 15 B & 11.06 & 1.79 & M6 Ve & 3.6 & 453 & 0.260 & 0.026 & 202 & 18 & -0.66 & 0.08 & 125.2 & 1.9 \\
\hline LHS 5004a & 12.29 & 1.46 & M3: & 20.0 & 209 & 1.001 & 0.071 & 633 & 49 & -0.17 & 0.07 & 741.6 & 353.5 \\
\hline LP 149-56 & 12.84 & & $\mathrm{~m}$ & 21.6 & 513 & 0.074 & 0.014 & 55 & 10 & -0.26 & 0.16 & 51.1 & 28.5 \\
\hline Gl 17.2 & 8.30 & 0.90 & K3 V & 27.4 & 239 & 0.051 & 0.018 & 11 & 22 & -0.26 & 0.34 & 35.1 & 31.6 \\
\hline Gl 17.3 & 6.38 & 0.66 & G2 V & 20.4 & 321 & 0.289 & 0.033 & 209 & 12 & -0.29 & 0.10 & 195.7 & 97.5 \\
\hline GJ $1010 \mathrm{~A}$ & 11.30 & 1.49 & $\mathrm{k}-\mathrm{m}$ & 19.7 & 914 & 0.011 & 0.004 & 10 & 14 & -0.61 & 0.33 & 5.7 & 2.7 \\
\hline GJ $1010 \mathrm{~B}$ & 14.00 & & $\mathrm{~m}$ & 19.7 & 914 & 0.011 & 0.004 & 10 & 23 & -0.61 & 0.33 & 5.7 & 2.7 \\
\hline GJ 2004 & 8.75 & 1.07 & $\mathrm{~K} 3 / 4 \mathrm{~V}$ & 22.2 & 328 & 0.033 & 0.012 & 13 & 11 & -1.00 & 0.64 & 9.8 & 5.8 \\
\hline G130-068 & 14.54 & 1.67 & $\mathrm{~m}$ & 18.9 & 321 & 0.065 & 0.016 & 38 & 13 & -0.43 & 0.22 & 39.1 & 16.8 \\
\hline GJ 2005 & 15.42 & & M5.5 & 7.4 & 212 & 0.053 & 0.018 & 13 & 62 & -0.56 & 0.27 & 28.3 & 1.8 \\
\hline Gl 19 & 2.80 & 0.62 & G2 IV & 7.5 & 195 & 0.070 & 0.023 & 15 & 21 & -1.00 & 0.41 & 21.0 & 1.4 \\
\hline G172-001 & 13.15 & & $\mathrm{~m}$ & 13.4 & 523 & 0.232 & 0.023 & 232 & 8 & -0.24 & 0.09 & 163.4 & 35.2 \\
\hline Gl $22 \mathrm{AC}$ & 10.38 & 1.54 & $\mathrm{dM} 2.5 \mathrm{e}$ & 10.1 & 304 & 0.085 & 0.020 & 33 & 18 & -0.44 & 0.18 & 50.6 & 6.2 \\
\hline Gl 22 B & 12.40 & & dM3.5 & 10.1 & 304 & 0.085 & 0.020 & 33 & 18 & -0.44 & 0.18 & 50.6 & 6.2 \\
\hline LP 525-39 & 12.70 & & $\mathrm{~m}$ & 11.8 & 133 & 0.287 & 0.050 & 77 & 13 & 0.01 & 0.18 & 240.0 & 39.9 \\
\hline Gl 22.2 & 5.57 & 0.46 & F3 IV-V & 25.6 & 216 & 0.037 & 0.015 & 12 & 40 & -1.00 & 2.36 & 11.1 & 8.8 \\
\hline Gl $23 \mathrm{~A}$ & 5.65 & 0.57 & F6 V & 21.1 & 421 & 1.116 & 0.076 & 588 & 8 & -0.21 & 0.07 & 803.2 & 425.9 \\
\hline Gl $23 \mathrm{~B}$ & 6.40 & & G1 V & 21.1 & 421 & 1.116 & 0.076 & 588 & 8 & -0.21 & 0.07 & 803.2 & 425.9 \\
\hline HD 3302 & 5.51 & 0.44 & F6 V & 36.2 & 184 & 0.224 & 0.039 & 61 & 19 & -0.22 & 0.16 & 160.2 & 251.7 \\
\hline $\mathrm{Gl} 24 \mathrm{~A}$ & 6.79 & 0.64 & G3 V & 43.9 & 231 & 0.327 & 0.039 & 168 & 5 & -0.37 & 0.12 & 207.8 & 478.3 \\
\hline Gl 28 & 7.36 & 0.94 & $\mathrm{~K} 2 \mathrm{Ve}$ & 17.3 & 485 & 0.026 & 0.009 & 13 & 30 & -0.45 & 0.31 & 15.4 & 5.5 \\
\hline Gl 29.1 & 10.52 & 1.50 & $\mathrm{dMO}$ e & 23.8 & 464 & 1.022 & 0.047 & 999 & 5 & -0.21 & 0.05 & 735.5 & 498.9 \\
\hline Gl 31 & 2.04 & 1.01 & K1 IIIe & 29.4 & 313 & 2.649 & 0.165 & 835 & 1 & 0.28 & 0.06 & 2594.4 & 2685.2 \\
\hline Gl 31.3 & 5.23 & 0.34 & $\mathrm{~F} 2 \mathrm{~V}$ & 27.9 & 338 & 0.217 & 0.028 & 144 & 13 & -0.07 & 0.12 & 172.3 & 159.9 \\
\hline $\mathrm{Gl} 32 \mathrm{~A}$ & 8.41 & 1.17 & K5 V & 16.0 & 296 & 0.037 & 0.014 & 8 & 36 & 0.04 & 0.42 & 31.2 & 9.6 \\
\hline Gl 32 B & 9.06 & 1.27 & K7 V & 16.0 & 296 & 0.037 & 0.014 & 8 & 38 & 0.04 & 0.42 & 31.2 & 9.6 \\
\hline GJ 1021 & 5.80 & 0.64 & G5 IV & 14.9 & 215 & 0.335 & 0.044 & 130 & 8 & -0.39 & 0.11 & 208.9 & 55.8 \\
\hline Gl 33 & 5.74 & 0.88 & $\mathrm{~K} 2 \mathrm{~V}$ & 7.5 & 353 & 0.043 & 0.013 & 17 & 31 & -1.00 & 0.20 & 13.0 & 0.9 \\
\hline Gl $34 \mathrm{~A}$ & 3.45 & 0.57 & G3 V & 6.0 & 381 & 0.138 & 0.022 & 71 & 12 & -0.72 & 0.11 & 62.0 & 2.6 \\
\hline Gl $34 \mathrm{~B}$ & 7.51 & 1.39 & $\mathrm{~K} 7 \mathrm{~V}$ & 6.0 & 381 & 0.138 & 0.022 & 71 & 12 & -0.72 & 0.11 & 62.0 & 2.6 \\
\hline LP $193-584$ & 13.06 & & $\mathrm{~m}$ & 19.3 & 185 & 0.222 & 0.037 & 80 & 24 & -0.24 & 0.17 & 156.5 & 69.8 \\
\hline Gl 34.1 & 5.07 & 0.51 & $\mathrm{~F} 8 \mathrm{~V}$ & 23.9 & 418 & 0.024 & 0.009 & 11 & 12 & -0.73 & 0.29 & 10.6 & 7.3 \\
\hline Gl 36 & 7.16 & 0.78 & G9 V & 18.8 & 311 & 0.034 & 0.012 & 11 & 33 & -0.90 & 0.24 & 12.1 & 5.2 \\
\hline Gl 37 & 5.17 & 0.50 & F7 IV-V & 15.5 & 565 & 0.073 & 0.013 & 54 & 4 & -0.72 & 0.12 & 32.7 & 9.4 \\
\hline LTT 10301 & 12.70 & & $\mathrm{~m}$ & 12.0 & 147 & 0.443 & 0.058 & 187 & 6 & -0.21 & 0.13 & 319.2 & 55.2 \\
\hline LP 350-19 & 13.30 & & $\mathrm{~m}$ & 12.0 & 147 & 0.443 & 0.058 & 187 & 6 & -0.21 & 0.13 & 319.2 & 55.2 \\
\hline Gl 39 & 9.24 & 1.21 & $\mathrm{dK} 6$ & 21.9 & 403 & 0.023 & 0.009 & 13 & 8 & 0.58 & 0.35 & 26.4 & 15.1 \\
\hline Gl 41 & 4.82 & 0.53 & $\mathrm{~F} 8 \mathrm{~V}$ & 18.6 & 326 & 0.044 & 0.014 & 12 & 29 & -0.71 & 0.24 & 19.9 & 8.2 \\
\hline Gl 42 & 7.17 & 0.93 & K3 V & 14.1 & 337 & 0.052 & 0.016 & 14 & 13 & -0.63 & 0.18 & 26.0 & 6.2 \\
\hline Gl 42.1 & 7.38 & 0.65 & G5 & 29.5 & 108 & 0.064 & 0.030 & 9 & 41 & -0.66 & 0.62 & 30.6 & 31.8 \\
\hline Gl 47 & 10.83 & 1.57 & $\mathrm{dM} 2.5 \mathrm{e}$ & 11.0 & 407 & 0.031 & 0.011 & 16 & 23 & -0.80 & 0.28 & 12.7 & 1.8 \\
\hline Gl 48 & 10.04 & 1.46 & $\mathrm{dM} 3.5 \mathrm{e}$ & 8.1 & 650 & 0.013 & 0.006 & 9 & 22 & -0.90 & 0.61 & 4.7 & 0.4 \\
\hline Gl 51 & 13.66 & 1.68 & M5 & 10.5 & 416 & 0.227 & 0.026 & 179 & 16 & -0.20 & 0.11 & 164.8 & 21.6 \\
\hline GJ $1026 \mathrm{~A}$ & 11.88 & & M1.5 & 16.3 & 291 & 0.146 & 0.024 & 98 & 9 & -0.27 & 0.15 & 100.6 & 32.1 \\
\hline GJ $1026 \mathrm{~B}$ & 12.40 & & M3.5 & 16.3 & 291 & 0.146 & 0.024 & 98 & 9 & -0.27 & 0.15 & 100.6 & 32.1 \\
\hline LP $467-16$ & 14.36 & & $\mathrm{~m}$ & 8.8 & 345 & 0.094 & 0.018 & 62 & 7 & -0.08 & 0.20 & 73.8 & 6.8 \\
\hline Gl 54.1 & 12.05 & 1.84 & dM5 e & 3.7 & 461 & 0.254 & 0.026 & 200 & 15 & -0.19 & 0.10 & 185.4 & 3.1 \\
\hline HD 7205 & 7.28 & 0.78 & G5 & 44.6 & 385 & 0.269 & 0.029 & 247 & 4 & -0.10 & 0.10 & 209.6 & 499.8 \\
\hline GJ 1033 & 14.16 & 1.58 & & 17.4 & 287 & 0.071 & 0.018 & 37 & 20 & -0.16 & 0.25 & 52.8 & 19.1 \\
\hline Gl $54.2 \mathrm{~A}$ & 5.14 & 0.45 & F5 V & 24.4 & 335 & 0.126 & 0.021 & 84 & 11 & -0.01 & 0.16 & 104.0 & 74.0 \\
\hline Gl 54.2B & 7.85 & 0.78 & $\mathrm{~K} 1 \mathrm{~V}$ & 24.4 & 335 & 0.126 & 0.021 & 84 & 55 & -0.01 & 0.16 & 104.0 & 74.0 \\
\hline Gl 55 & 4.96 & 0.58 & F8 V & 15.1 & 324 & 0.039 & 0.013 & 16 & 9 & -1.00 & 0.98 & 11.6 & 3.2 \\
\hline Gl $55.1 \mathrm{~A}$ & 7.80 & 0.98 & $\mathrm{~K} 2 \mathrm{~V}$ & 21.1 & 107 & 0.115 & 0.039 & 13 & 25 & -0.11 & 0.31 & 89.1 & 47.4 \\
\hline Gl 55.1B & 8.20 & & & 21.1 & 107 & 0.115 & 0.039 & 13 & 25 & -0.11 & 0.31 & 89.1 & 47.4 \\
\hline Gl 55.3A & 4.99 & 0.47 & F6 IV & 20.4 & 110 & 0.522 & 0.072 & 116 & 15 & -0.40 & 0.13 & 322.9 & 161.6 \\
\hline Gl 55.3B & 7.20 & & G5 & 20.4 & 110 & 0.522 & 0.072 & 116 & 10 & -0.40 & 0.13 & 322.9 & 161.6 \\
\hline G269-139 & 14.20 & & $\mathrm{~m}$ & 24.3 & 424 & 0.066 & 0.017 & 13 & 54 & -0.14 & 0.30 & 49.9 & 35.2 \\
\hline Gl 56.5 & 7.11 & 0.82 & dK0 & 16.8 & 786 & 0.014 & 0.005 & 9 & 29 & -0.49 & 0.32 & 7.9 & 2.7 \\
\hline HD 8357 & 7.33 & & G5 & 45.2 & 396 & 4.673 & 0.358 & 546 & 5 & -0.17 & 0.08 & 3462.2 & 8481.2 \\
\hline Gl 57.1A & 7.85 & 0.90 & K0 IV & 30.2 & 390 & 0.022 & 0.009 & 10 & 9 & -0.89 & 0.25 & 8.0 & 8.8 \\
\hline
\end{tabular}




\begin{tabular}{|c|c|c|c|c|c|c|c|c|c|c|c|c|c|}
\hline Name & $V$ & $B-V$ & Sp. type & $d(\mathrm{pc})$ & Exp. & $C R$ & $\pm C R$ & $\mathrm{Li}$ & $\Delta$ & $h r$ & $\Delta h r$ & $f_{\mathrm{x}-14}$ & $L_{\mathrm{x} 27}$ \\
\hline Gl 57.1B & 10.38 & 1.38 & $\mathrm{dK} 7$ & 30.2 & 390 & 0.022 & 0.009 & 10 & 46 & -0.89 & 0.25 & 8.0 & 8.8 \\
\hline Gl $57.1 \mathrm{C}$ & 13.10 & & & 30.2 & 390 & 0.022 & 0.009 & 10 & 46 & -0.89 & 0.25 & 8.0 & 8.8 \\
\hline Gl 58.2 & 7.73 & 0.96 & $\mathrm{~K} 2 \mathrm{~V}$ & 23.1 & 436 & 0.105 & 0.017 & 69 & 8 & -0.41 & 0.14 & 64.4 & 41.3 \\
\hline Gl $59 \mathrm{~A}$ & 6.97 & 0.76 & G8 V & 19.5 & 251 & 0.059 & 0.019 & 19 & 4 & -0.80 & 0.17 & 24.1 & 10.9 \\
\hline Gl $60 \mathrm{~A}$ & 7.78 & 0.92 & K3 V & 23.6 & 486 & 2.594 & 0.209 & 389 & 6 & -0.20 & 0.08 & 1880.7 & 1257.5 \\
\hline Gl $60 \mathrm{~B}$ & 8.00 & & $\mathrm{~K} 4 \mathrm{~V}$ & 23.6 & 486 & 2.594 & 0.209 & 389 & 6 & -0.20 & 0.08 & 1880.7 & 1257.5 \\
\hline Gl $60 \mathrm{C}$ & 10.40 & & M2 V & 23.6 & 486 & 2.594 & 0.209 & 389 & 6 & -0.20 & 0.08 & 1880.7 & 1257.5 \\
\hline Gl 61 & 4.09 & 0.54 & F8 V & 13.5 & 354 & 0.135 & 0.023 & 66 & 33 & -0.71 & 0.11 & 61.3 & 13.3 \\
\hline LHS 1268 & 10.50 & & $\mathrm{~K} 7$ & 21.8 & 257 & 0.142 & 0.027 & 57 & 30 & -0.03 & 0.19 & 115.6 & 65.9 \\
\hline Gl $65 \mathrm{~A}$ & 12.57 & 1.85 & $\mathrm{dM} 5.5 \mathrm{e}$ & 2.7 & 484 & 0.680 & 0.057 & 318 & 11 & -0.32 & 0.08 & 450.0 & 3.9 \\
\hline Gl $65 \mathrm{~B}$ & 12.70 & & $\mathrm{dM} 5.5 \mathrm{e}$ & 2.7 & 484 & 0.680 & 0.057 & 318 & 11 & -0.32 & 0.08 & 450.0 & 3.9 \\
\hline Gl 65.2 & 9.86 & 1.16 & M0 & 40.2 & 423 & 0.022 & 0.010 & 9 & 29 & -0.78 & 0.24 & 9.2 & 17.7 \\
\hline Gl 67.1 & 5.88 & 0.61 & $\mathrm{G} 2 \mathrm{~V}$ & 27.1 & 291 & 0.260 & 0.032 & 156 & 6 & -0.09 & 0.12 & 203.3 & 178.7 \\
\hline Gl 69 & 8.41 & 1.22 & K5 V & 13.4 & 533 & 0.056 & 0.012 & 49 & 15 & -0.41 & 0.19 & 34.6 & 7.5 \\
\hline Gl 71 & 3.49 & 0.72 & G8 Vp & 3.6 & 445 & 0.052 & 0.013 & 24 & 14 & -1.00 & 0.09 & 15.6 & 0.2 \\
\hline Gl 75 & 5.63 & 0.81 & $\mathrm{~K} 0 \mathrm{~V}$ & 10.0 & 572 & 0.360 & 0.026 & 505 & 6 & -0.60 & 0.06 & 184.8 & 22.0 \\
\hline Wo $9061 \mathrm{~A}$ & 4.67 & 0.33 & F3 III & 23.6 & 454 & 0.248 & 0.025 & 211 & 4 & -0.39 & 0.09 & 155.0 & 103.7 \\
\hline Wo $9061 \mathrm{~B}$ & 6.77 & 0.62 & dG1 & 23.0 & 450 & 0.386 & 0.032 & 430 & 5 & -0.39 & 0.07 & 241.0 & 152.4 \\
\hline G159-003 & 14.60 & & $\mathrm{~m}$ & 10.7 & 230 & 0.050 & 0.018 & 15 & 19 & -0.36 & 0.29 & 32.0 & 4.4 \\
\hline Gl 78.1 & 3.42 & 0.48 & F6 IV & 19.6 & 284 & 1.005 & 0.062 & 875 & 11 & -0.05 & 0.06 & 808.5 & 373.4 \\
\hline HD 11373 & 8.48 & 1.01 & G5 & 22.1 & 509 & 0.040 & 0.010 & 29 & 25 & -0.11 & 0.25 & 30.9 & 18.1 \\
\hline Gl 79 & 8.90 & 1.41 & K5/M0 V & 11.1 & 395 & 0.070 & 0.016 & 34 & 14 & -0.30 & 0.20 & 47.0 & 6.9 \\
\hline Gl $81 \mathrm{~A}$ & 3.70 & 0.85 & G5 IV & 17.5 & 280 & 1.660 & 0.115 & 579 & 3 & 0.00 & 0.07 & 1379.5 & 504.4 \\
\hline Gl $81 \mathrm{~B}$ & 10.70 & & & 17.5 & 280 & 1.660 & 0.115 & 579 & 3 & 0.00 & 0.07 & 1379.5 & 504.4 \\
\hline Gl 82 & 12.21 & 1.56 & $\mathrm{dM} 4 \mathrm{e}$ & 12.0 & 583 & 0.457 & 0.029 & 670 & 6 & -0.23 & 0.06 & 323.8 & 56.0 \\
\hline GJ $1041 \mathrm{~A}$ & 10.98 & 1.52 & $\mathrm{k}-\mathrm{m}$ & 29.9 & 387 & 0.324 & 0.031 & 261 & 8 & -0.24 & 0.09 & 227.8 & 242.9 \\
\hline GJ $1041 \mathrm{~B}$ & 14.00 & & $\mathrm{~m}$ & 29.9 & 387 & 0.324 & 0.031 & 261 & 8 & -0.24 & 0.09 & 227.8 & 242.9 \\
\hline Gl 83 & 2.86 & 0.28 & $\mathrm{~F} 0 \mathrm{~V}$ & 21.9 & 378 & 0.055 & 0.015 & 21 & 4 & -1.00 & 0.08 & 16.6 & 9.5 \\
\hline G245-040 & 14.12 & 1.90 & $\mathrm{~m}$ & 9.5 & 765 & 0.044 & 0.009 & 38 & 20 & -0.48 & 0.16 & 25.4 & 2.7 \\
\hline Gl 83.1 & 12.28 & 1.80 & dM8 e & 4.4 & 295 & 0.167 & 0.025 & 111 & 7 & -0.27 & 0.15 & 115.2 & 2.7 \\
\hline G272-137 & 14.10 & & $\mathrm{~m}$ & 24.2 & 325 & 0.025 & 0.010 & 11 & 32 & -0.47 & 0.29 & 14.4 & 10.1 \\
\hline G003-035 & 14.27 & & dM5 & 16.8 & 289 & 0.126 & 0.023 & 70 & 5 & -0.42 & 0.17 & 76.6 & 25.7 \\
\hline HD 12545 & 8.36 & & G5 & 196.1 & 117 & 0.995 & 0.096 & 247 & 3 & 0.36 & 0.09 & 1017.2 & 46790.3 \\
\hline $\mathrm{Gl} 83.4 \mathrm{~A}$ & 7.31 & 0.69 & G3 V & 49.3 & 471 & 0.431 & 0.032 & 442 & 2 & 0.12 & 0.07 & 385.1 & 1118.1 \\
\hline $\mathrm{Gl} 83.4 \mathrm{~B}$ & 11.50 & & & 49.3 & 471 & 0.431 & 0.032 & 442 & 2 & 0.12 & 0.07 & 385.1 & 1118.1 \\
\hline HD 12786 & 7.78 & & $\mathrm{~K} 0 \mathrm{~V}$ & 25.5 & 234 & 0.100 & 0.024 & 30 & 18 & 0.31 & 0.24 & 99.2 & 77.3 \\
\hline Gl 84 & 10.19 & 1.51 & M3 & 9.4 & 374 & 0.077 & 0.017 & 38 & 24 & -0.52 & 0.18 & 42.9 & 4.6 \\
\hline G244-049 & 14.31 & & $\mathrm{~m}$ & 19.3 & 522 & 0.088 & 0.015 & 61 & 13 & -0.25 & 0.16 & 61.3 & 27.3 \\
\hline *G173-039 & 12.47 & 1.54 & M5 & 14.2 & 694 & 0.210 & 0.017 & 489 & 10 & -0.26 & 0.07 & 145.9 & 35.2 \\
\hline Gl 86 & 6.12 & 0.82 & $\mathrm{~K} 0 \mathrm{~V}$ & 10.9 & 375 & 0.058 & 0.016 & 24 & 28 & -0.64 & 0.19 & 28.6 & 4.1 \\
\hline Gl $87.1 \mathrm{~A}$ & 5.67 & 0.56 & $\mathrm{~F} 8 \mathrm{~V}$ & 46.1 & 345 & 0.031 & 0.012 & 8 & 30 & -0.47 & 0.32 & 17.9 & 45.5 \\
\hline Gl $87.1 \mathrm{~B}$ & 7.74 & 0.68 & G5 V & 25.6 & 345 & 0.031 & 0.012 & 8 & 45 & -0.47 & 0.32 & 17.9 & 14.1 \\
\hline G159-046 & 13.50 & & $\mathrm{~m}$ & 14.1 & 316 & 0.085 & 0.019 & 42 & 7 & -0.24 & 0.21 & 59.6 & 14.2 \\
\hline GJ 1044 & 9.88 & 1.36 & $\mathrm{~K} 7 \mathrm{~V}$ & 23.3 & 278 & 0.024 & 0.011 & 7 & 50 & 0.17 & 0.54 & 21.8 & 14.1 \\
\hline Gl 90 & 7.09 & 0.90 & $\mathrm{~K} 2 \mathrm{~V}$ & 18.1 & 672 & 0.045 & 0.009 & 44 & 3 & -0.62 & 0.14 & 22.8 & 9.0 \\
\hline GJ 1045 & 14.44 & 1.62 & $\mathrm{~m}+$ & 20.5 & 184 & 0.019 & 0.011 & 8 & 67 & & & 16.0 & 8.1 \\
\hline G074-011 & 13.58 & & $\mathrm{~m}$ & 18.0 & 270 & 0.044 & 0.017 & 9 & 38 & -0.20 & 0.35 & 31.8 & 12.2 \\
\hline $\mathrm{Gl} 91.2 \mathrm{~A}$ & 8.42 & 1.02 & $\mathrm{~K} 3 \mathrm{~V}$ & 22.2 & 389 & 0.084 & 0.017 & 40 & 13 & -0.28 & 0.18 & 57.6 & 34.0 \\
\hline Gl $91.2 \mathrm{~B}$ & 9.10 & & $\mathrm{~K}$ & 22.2 & 389 & 0.084 & 0.017 & 40 & 13 & -0.28 & 0.18 & 57.6 & 34.0 \\
\hline LHS 1375 & 15.79 & 1.98 & $\mathrm{~m}$ & 8.5 & 229 & 0.035 & 0.014 & 14 & 41 & -0.52 & 0.43 & 19.3 & 1.7 \\
\hline Gl 92 & 4.87 & 0.61 & G0 Ve & 10.8 & 299 & 0.423 & 0.039 & 285 & 8 & -0.38 & 0.09 & 266.6 & 37.5 \\
\hline LHS 1377 & 12.00 & & M4 & 14.2 & 481 & 0.242 & 0.024 & 189 & 11 & -0.52 & 0.08 & 134.6 & 32.7 \\
\hline G035-040 & 14.17 & & $\mathrm{~m}$ & 22.8 & 200 & 0.057 & 0.021 & 15 & 29 & 0.33 & 0.35 & 57.5 & 35.7 \\
\hline HD 14629 & 8.74 & 1.04 & $\mathrm{~K} 3 / 4 \mathrm{~V}$ & 25.4 & 517 & 0.021 & 0.009 & 9 & 5 & -0.35 & 0.31 & 13.9 & 10.8 \\
\hline Gl 96 & 9.41 & 1.49 & $\mathrm{dM} 1.5 \mathrm{e}$ & 11.9 & 563 & 0.031 & 0.009 & 19 & 17 & -0.58 & 0.22 & 16.2 & 2.8 \\
\hline Gl 97 & 5.20 & 0.60 & G1 V & 21.9 & 266 & 0.869 & 0.060 & 726 & 11 & -0.08 & 0.07 & 685.5 & 394.4 \\
\hline G074-025 & 14.05 & & $\mathrm{~m}$ & 19.6 & 305 & 0.050 & 0.015 & 16 & 11 & -0.50 & 0.28 & 28.3 & 13.1 \\
\hline HD 15814 & 6.02 & 0.54 & $\mathrm{~F} 8 \mathrm{~V}$ & 28.7 & 122 & 0.306 & 0.055 & 58 & 19 & -0.39 & 0.16 & 191.3 & 189.0 \\
\hline Gl 102 & 12.96 & 1.70 & M4 & 9.8 & 246 & 0.145 & 0.027 & 51 & 15 & -0.52 & 0.15 & 80.6 & 9.2 \\
\hline Gl 103 & 8.85 & 1.39 & $\mathrm{~K} 7 \mathrm{Ve}$ & 11.5 & 460 & 2.705 & 0.224 & 360 & 11 & 0.17 & 0.08 & 2491.6 & 394.8 \\
\hline $023230+2321$. & 13.71 & 1.58 & M3 e & 18.2 & 243 & 0.098 & 0.023 & 34 & 40 & -0.42 & 0.21 & 59.7 & 23.7 \\
\hline Gl $105 \mathrm{~A}$ & 5.82 & 0.98 & $\mathrm{~K} 3 \mathrm{~V}$ & 7.2 & 232 & 0.058 & 0.019 & 19 & 15 & -0.66 & 0.20 & 28.1 & 1.7 \\
\hline GJ 1048 & 8.43 & 1.08 & $\mathrm{~K} 2 \mathrm{~V}$ & 21.3 & 338 & 0.036 & 0.013 & 16 & 15 & -0.11 & 0.35 & 27.7 & 15.0 \\
\hline G074-034 & 13.63 & & M3 & 15.3 & 317 & 0.048 & 0.015 & 20 & 22 & -0.70 & 0.20 & 22.3 & 6.3 \\
\hline LTT 10855 & 13.90 & & M3.5 & 15.3 & 317 & 0.048 & 0.015 & 20 & 22 & -0.70 & 0.20 & 22.3 & 6.3 \\
\hline Wo 9087 & 8.10 & 0.95 & K3 & 24.3 & 105 & 0.115 & 0.038 & 17 & 64 & -0.07 & 0.31 & 91.7 & 64.9 \\
\hline $023424+0641$. & 16.00 & & M5 e & 20.3 & 226 & 0.034 & 0.016 & 9 & 73 & -0.15 & 0.43 & 25.9 & 12.8 \\
\hline Gl 105.1 & 5.78 & 0.65 & G5 IV & 25.5 & 448 & 0.028 & 0.010 & 12 & 4 & -1.00 & 0.22 & 8.4 & 6.5 \\
\hline G076-019 & 14.27 & & $\mathrm{~m}$ & 20.7 & 243 & 0.054 & 0.018 & 14 & 5 & -0.09 & 0.33 & 42.3 & 21.6 \\
\hline Gl $105.4 \mathrm{~A}$ & 5.50 & 0.44 & F5 V & 27.0 & 256 & 0.053 & 0.018 & 13 & 7 & -0.45 & 0.28 & 31.2 & 27.2 \\
\hline Gl $105.4 \mathrm{~B}$ & 5.60 & & F5 V & 27.0 & 256 & 0.053 & 0.018 & 13 & 7 & -0.45 & 0.28 & 31.2 & 27.2 \\
\hline GJ 1049 & 9.65 & 1.39 & M0 Ve & 16.5 & 272 & 1.337 & 0.073 & 938 & 4 & -0.21 & 0.05 & 962.2 & 314.5 \\
\hline HD 16673 & 5.78 & 0.52 & F8 V & 21.6 & 225 & 0.156 & 0.030 & 54 & 16 & -0.81 & 0.10 & 62.6 & 34.8 \\
\hline Gl 105.6 & 4.92 & 0.59 & F9 V & 24.7 & 558 & 0.034 & 0.010 & 15 & 23 & -0.57 & 0.26 & 17.8 & 13.0 \\
\hline Gl 106 & 8.28 & 1.07 & $\mathrm{dK} 4$ & 18.6 & 302 & 0.081 & 0.019 & 40 & 16 & -0.20 & 0.21 & 58.7 & 24.2 \\
\hline Gl $106.1 \mathrm{~A}$ & 3.56 & 0.09 & A3 V & 25.1 & 224 & 0.376 & 0.044 & 159 & 20 & -0.12 & 0.12 & 288.8 & 218.2 \\
\hline Gl $106.1 \mathrm{~B}$ & 6.30 & & dF3 & 25.1 & 224 & 0.376 & 0.044 & 159 & 20 & -0.12 & 0.12 & 288.8 & 218.2 \\
\hline Gl $107 \mathrm{~A}$ & 4.13 & 0.49 & F7 V & 11.2 & 527 & 0.152 & 0.019 & 140 & 6 & -0.70 & 0.08 & 70.1 & 10.6 \\
\hline Gl $107 \mathrm{~B}$ & 10.06 & 1.48 & M1 V & 11.2 & 527 & 0.152 & 0.019 & 140 & 6 & -0.70 & 0.08 & 70.1 & 10.6 \\
\hline Gl 108 & 5.41 & 0.56 & G3 IV & 17.2 & 825 & 0.281 & 0.045 & 70 & 9 & -0.29 & 0.14 & 190.5 & 67.8 \\
\hline
\end{tabular}




\begin{tabular}{|c|c|c|c|c|c|c|c|c|c|c|c|c|c|}
\hline Name & $V$ & $B-V$ & Sp. type & $d(\mathrm{pc})$ & Exp. & $C R$ & $\pm C R$ & $\mathrm{Li}$ & $\Delta$ & $h r$ & $\Delta h r$ & $f_{\mathrm{x}-14}$ & $L_{\mathrm{x} 27}$ \\
\hline Gl 109 & 10.57 & 1.56 & dM3.5 & 7.6 & 399 & 0.053 & 0.014 & 23 & 17 & -0.51 & 0.27 & 29.6 & 2.0 \\
\hline HD 17169 & 7.12 & & G5 V & 107.5 & 401 & 0.098 & 0.018 & 67 & 10 & 0.18 & 0.18 & 90.4 & 1250.8 \\
\hline *Wo 9099 & 4.27 & 0.31 & F0 IV & 25.8 & 1263 & 0.101 & 0.009 & 295 & 21 & -0.64 & 0.07 & 49.7 & 39.7 \\
\hline Gl 111 & 4.46 & 0.48 & F6 V & 14.0 & 288 & 0.906 & 0.059 & 559 & 11 & -0.23 & 0.06 & 642.7 & 150.0 \\
\hline Gl $113 \mathrm{AB}$ & 7.61 & 0.83 & $\mathrm{~K} 1 \mathrm{Ve}$ & 22.4 & 280 & 0.116 & 0.023 & 54 & 18 & -0.26 & 0.17 & 80.8 & 48.4 \\
\hline Gl $113 \mathrm{C}$ & 16.50 & & $\mathrm{~m}$ & 22.4 & 280 & 0.116 & 0.023 & 54 & 24 & -0.26 & 0.17 & 80.8 & 48.4 \\
\hline Gl 113.1 & 6.76 & 0.96 & G9 e & 44.1 & 169 & 6.792 & 0.202 & 999 & 6 & -0.08 & 0.03 & 5356.2 & 12436.3 \\
\hline LP 298-42 & 13.96 & & $\mathrm{~m}$ & 16.2 & 229 & 0.041 & 0.016 & 14 & 16 & -0.50 & 0.32 & 22.9 & 7.2 \\
\hline Gl 117 & 6.03 & 0.87 & $\mathrm{~K} 2 \mathrm{~V}$ & 10.4 & 220 & 1.200 & 0.077 & 883 & 13 & -0.11 & 0.06 & 927.2 & 119.6 \\
\hline HD 17948 & 5.59 & 0.45 & $\mathrm{~F} 4 \mathrm{~V}$ & 26.5 & 550 & 0.028 & 0.009 & 18 & 32 & -1.00 & 0.26 & 8.5 & 7.1 \\
\hline Gl $118.1 \mathrm{~A}$ & 8.23 & 0.93 & K3 V & 29.6 & 408 & 0.073 & 0.016 & 34 & 12 & -0.65 & 0.14 & 35.3 & 37.0 \\
\hline Gl 118.1B & 13.10 & 1.52 & M3 : & 29.6 & 408 & 0.073 & 0.016 & 34 & 25 & -0.65 & 0.14 & 35.3 & 37.0 \\
\hline Gl $120.1 \mathrm{~A}$ & 8.03 & 0.87 & $\mathrm{~K} 1 / 2 \mathrm{~V}$ & 22.5 & 262 & 0.029 & 0.012 & 10 & 24 & -1.00 & 1.23 & 8.7 & 5.3 \\
\hline Gl 120.1B & 8.20 & & & 22.5 & 262 & 0.029 & 0.012 & 10 & 24 & -1.00 & 1.23 & 8.7 & 5.3 \\
\hline Gl $120.1 \mathrm{C}$ & 7.83 & 0.95 & $\mathrm{~K} 2 \mathrm{~V}$ & 25.7 & 262 & 0.029 & 0.012 & 10 & 21 & -1.00 & 1.23 & 8.7 & 6.9 \\
\hline Gl 120.2 & 6.62 & 0.72 & G8 V & 21.1 & 592 & 0.020 & 0.008 & 10 & 33 & -1.00 & 0.20 & 6.0 & 3.2 \\
\hline LTT 1445 & 10.96 & 1.69 & M3 & 7.6 & 211 & 0.177 & 0.033 & 57 & 26 & -0.54 & 0.15 & 96.4 & 6.7 \\
\hline LP 771-96 & 11.80 & & M3 & 7.6 & 211 & 0.177 & 0.033 & 57 & 26 & -0.54 & 0.15 & 96.4 & 6.7 \\
\hline Gl 124 & 4.05 & 0.60 & G0 V & 10.5 & 523 & 0.021 & 0.007 & 13 & 57 & -1.00 & 0.89 & 6.4 & 0.9 \\
\hline GJ $1054 \mathrm{~A}$ & 10.24 & 1.41 & K7 V & 19.1 & 227 & 1.538 & 0.086 & 999 & 8 & -0.28 & 0.05 & 1049.8 & 457.5 \\
\hline GJ 1054 B & 13.09 & 1.64 & $\mathrm{~m}+$ & 19.1 & 227 & 1.538 & 0.086 & 999 & 65 & -0.28 & 0.05 & 1049.8 & 457.5 \\
\hline CD-25 1273 & 9.50 & & $\mathrm{~m}$ & 31.2 & 198 & 1.022 & 0.075 & 553 & 37 & -0.08 & 0.07 & 805.9 & 935.8 \\
\hline Gl 125 & 10.15 & 1.49 & $\mathrm{dM} 1$ & 15.4 & 510 & 0.015 & 0.007 & 7 & 4 & -0.51 & 0.33 & 8.7 & 2.5 \\
\hline G246-026 & 11.72 & 1.50 & M3 & 21.2 & 504 & 0.030 & 0.009 & 17 & 7 & -0.36 & 0.27 & 19.5 & 10.5 \\
\hline $\mathrm{BD}+60637$ & 10.12 & 1.32 & $\mathrm{dK} 8$ & 36.9 & 602 & 0.134 & 0.017 & 138 & 74 & -0.18 & 0.12 & 98.7 & 160.8 \\
\hline Gl $127 \mathrm{~A}$ & 3.95 & 0.51 & F7 IV & 14.1 & 212 & 2.792 & 0.168 & 916 & 6 & -0.09 & 0.06 & 2187.0 & 520.5 \\
\hline Gl $127 \mathrm{~B}$ & 6.70 & & G7 V & 14.1 & 212 & 2.792 & 0.168 & 916 & 6 & -0.09 & 0.06 & 2187.0 & 520.5 \\
\hline G078-028 & 12.39 & & $\mathrm{~m}$ & 15.2 & 498 & 0.014 & 0.007 & 8 & 48 & -0.05 & 0.46 & 11.3 & 3.1 \\
\hline Gl 135 & 7.03 & 0.66 & G1.5 V & 24.7 & 562 & 0.018 & 0.007 & 10 & 31 & -1.00 & 0.32 & 5.5 & 4.0 \\
\hline Gl 136 & 5.54 & 0.64 & G2 V & 12.1 & 328 & 0.095 & 0.020 & 35 & 15 & -0.91 & 0.09 & 33.1 & 5.8 \\
\hline Gl 137 & 4.82 & 0.68 & G5 Ve & 9.2 & 286 & 1.201 & 0.095 & 472 & 9 & -0.36 & 0.07 & 768.9 & 77.1 \\
\hline Gl 139 & 4.26 & 0.71 & G5 V & 6.1 & 519 & 0.026 & 0.009 & 10 & 13 & -0.76 & 0.31 & 11.1 & 0.5 \\
\hline G077-046 & 11.37 & 1.50 & M2 & 15.1 & 455 & 0.026 & 0.010 & 8 & 15 & -1.00 & 0.45 & 7.9 & 2.1 \\
\hline G005-032 & 12.19 & 1.56 & $\mathrm{~m}$ & 18.3 & 540 & 0.103 & 0.016 & 82 & 9 & -0.20 & 0.14 & 74.4 & 29.7 \\
\hline Gl $140 \mathrm{~A}$ & 10.64 & 1.51 & dM0 & 19.8 & 459 & 0.509 & 0.035 & 597 & 12 & -0.15 & 0.07 & 382.8 & 179.6 \\
\hline Gl $140 \mathrm{~B}$ & 12.00 & & & 19.8 & 459 & 0.509 & 0.035 & 597 & 12 & -0.15 & 0.07 & 382.8 & 179.6 \\
\hline Gl 140.1A & 8.47 & 1.13 & K5 V & 19.8 & 593 & 0.018 & 0.007 & 11 & 3 & & & 14.6 & 6.8 \\
\hline Gl 140.1B & 10.32 & 1.39 & $\mathrm{k}$ & 19.9 & 593 & 0.018 & 0.007 & 11 & 12 & & & 14.6 & 6.9 \\
\hline HD 21175 & 6.92 & & $\mathrm{~K} 0 \mathrm{~V}$ & 17.1 & 394 & 0.422 & 0.036 & 356 & 7 & -0.22 & 0.08 & 301.2 & 105.3 \\
\hline Gl 141 & 7.86 & 1.16 & K5 V & 15.1 & 375 & 0.032 & 0.012 & 13 & 4 & -0.21 & 0.39 & 22.9 & 6.2 \\
\hline LP $532-81$ & 14.70 & & $\mathrm{~m}+$ & 23.0 & 508 & 0.025 & 0.009 & 15 & 3 & -0.48 & 0.27 & 14.6 & 9.3 \\
\hline *Gl 142 & 8.39 & 1.33 & $\mathrm{~K} 7 \mathrm{~V}$ & 12.6 & 2017 & 0.091 & 0.007 & 457 & 16 & -0.49 & 0.07 & 52.0 & 9.9 \\
\hline LP $772-72$ & 14.28 & & $\mathrm{~m}$ & 21.9 & 185 & 0.060 & 0.022 & 10 & 24 & -0.45 & 0.35 & 35.4 & 20.3 \\
\hline LDS $3498 ?$ & 14.37 & & $\mathrm{~m}$ & 21.9 & 185 & 0.060 & 0.022 & 10 & 32 & -0.45 & 0.35 & 35.4 & 20.3 \\
\hline GJ $1060 \mathrm{~A}$ & 14.00 & & DA5 & 17.4 & 96 & 0.067 & 0.031 & 10 & 71 & 0.21 & 0.47 & 62.9 & 22.7 \\
\hline GJ $1060 \mathrm{~B}$ & 13.80 & & sdM3 & 17.4 & 96 & 0.067 & 0.031 & 10 & 67 & 0.21 & 0.47 & 62.9 & 22.7 \\
\hline Gl 143.1 & 9.98 & 1.42 & M0 V & 21.6 & 590 & 0.021 & 0.008 & 10 & 5 & -0.47 & 0.31 & 12.0 & 6.7 \\
\hline HD $21663 \mathrm{~A}$ & 8.32 & 0.74 & G5 & 46.3 & 426 & 0.018 & 0.008 & 7 & 34 & -0.91 & 0.66 & 6.3 & 16.2 \\
\hline HD $21663 \mathrm{~B}$ & 10.75 & 1.42 & dK6 & 46.3 & 426 & 0.018 & 0.008 & 7 & 34 & -0.91 & 0.66 & 6.3 & 16.2 \\
\hline Нy 207 & 10.79 & 1.39 & $\mathrm{~K} 7 \mathrm{~V}$ & 16.0 & 426 & 0.018 & 0.008 & 7 & 33 & -0.91 & 0.66 & 6.3 & 1.9 \\
\hline Gl 143.2A & 4.71 & 0.39 & F5 IV-V & 21.4 & 356 & 0.071 & 0.017 & 32 & 16 & -0.50 & 0.19 & 40.0 & 22.0 \\
\hline Gl 143.2B & 10.75 & 1.42 & $\mathrm{~m}$ & 21.4 & 356 & 0.071 & 0.017 & 32 & 61 & -0.50 & 0.19 & 40.0 & 22.0 \\
\hline Gl 144 & 3.73 & 0.88 & $\mathrm{~K} 2 \mathrm{~V}$ & 3.2 & 374 & 2.822 & 0.233 & 327 & 7 & -0.45 & 0.07 & 1672.0 & 20.7 \\
\hline Gl 145 & 11.48 & 1.57 & M3.5 & 10.9 & 412 & 0.030 & 0.011 & 8 & 17 & -0.72 & 0.28 & 13.3 & 1.9 \\
\hline LP 54-19 & 14.17 & & $\mathrm{~m}$ & 22.5 & 555 & 0.076 & 0.013 & 64 & 5 & -0.12 & 0.16 & 58.7 & 35.6 \\
\hline Gl 146 & 8.60 & 1.31 & $\mathrm{~K} 7 \mathrm{~V}$ & 13.4 & 555 & 0.030 & 0.010 & 9 & 22 & -0.78 & 0.19 & 12.3 & 2.6 \\
\hline Rob 233 & 13.86 & & dM5 : & 13.9 & 369 & 0.051 & 0.015 & 20 & 22 & -0.49 & 0.23 & 29.0 & 6.6 \\
\hline LP $413-18$ & 12.74 & & $\mathrm{~g}-\mathrm{k}$ & 23.3 & 381 & 0.141 & 0.021 & 91 & 31 & -0.10 & 0.15 & 109.5 & 70.9 \\
\hline LP 413-19 & 13.29 & & $\mathrm{~g}-\mathrm{k}$ & 23.3 & 381 & 0.141 & 0.021 & 91 & 13 & -0.10 & 0.15 & 109.5 & 70.9 \\
\hline LTT 11203 & 12.79 & & dM3.1 & 16.9 & 390 & 0.070 & 0.016 & 27 & 8 & -0.52 & 0.19 & 39.1 & 13.4 \\
\hline Wo 9119 A & 9.07 & 1.06 & $\mathrm{dK} 5$ & 50.0 & 402 & 0.160 & 0.021 & 127 & 14 & -0.10 & 0.14 & 124.7 & 373.0 \\
\hline Wo 9119 B & 12.87 & 1.49 & & 50.0 & 402 & 0.160 & 0.021 & 127 & 6 & -0.10 & 0.14 & 124.7 & 373.0 \\
\hline Gl 150 & 3.53 & 0.92 & K0 IVe & 9.0 & 532 & 0.019 & 0.008 & 9 & 26 & -0.66 & 0.29 & 9.2 & 0.9 \\
\hline Wo 9124 & 10.66 & 0.66 & F8 & 123.5 & 382 & 0.127 & 0.021 & 81 & 74 & 0.62 & 0.14 & 147.6 & 2691.9 \\
\hline Wo 9125 & 10.81 & 0.70 & G0 & 125.0 & 382 & 0.127 & 0.021 & 81 & 66 & 0.62 & 0.14 & 147.6 & 2759.6 \\
\hline Wo 9127 & 11.09 & 0.86 & G3 & 101.0 & 382 & 0.127 & 0.021 & 81 & 83 & 0.62 & 0.14 & 147.6 & 1802.0 \\
\hline Gl 152 & 6.99 & 0.88 & $\mathrm{~K} 0 \mathrm{~V}$ & 16.2 & 171 & 0.108 & 0.032 & 16 & 8 & -0.73 & 0.16 & 47.8 & 15.1 \\
\hline Gl $153 \mathrm{~A}$ & 9.33 & 1.28 & $\mathrm{~K} 7$ & 17.4 & 542 & 0.049 & 0.011 & 36 & 41 & -0.39 & 0.21 & 30.9 & 11.2 \\
\hline Gl $153 \mathrm{~B}$ & 11.30 & 1.54 & M2.5 & 17.4 & 542 & 0.049 & 0.011 & 36 & 24 & -0.39 & 0.21 & 30.9 & 11.2 \\
\hline Gl $153 \mathrm{C}$ & 11.60 & & & 17.4 & 542 & 0.049 & 0.011 & 36 & 24 & -0.39 & 0.21 & 30.9 & 11.2 \\
\hline Gl 154 & 9.61 & 1.47 & K7 & 14.6 & 367 & 0.036 & 0.012 & 17 & 9 & -0.66 & 0.24 & 17.6 & 4.5 \\
\hline Gl 155 & 4.22 & 0.42 & F3 III & 17.9 & 518 & 0.022 & 0.009 & 8 & 19 & -1.00 & 0.14 & 6.7 & 2.6 \\
\hline Wo 9135 & 10.23 & 1.16 & $\mathrm{dK} 6$ & 40.5 & 359 & 0.025 & 0.010 & 14 & 8 & -0.06 & 0.39 & 20.1 & 39.5 \\
\hline HD 24496 & 6.81 & & G5 & 20.7 & 330 & 0.057 & 0.015 & 32 & 2 & -0.39 & 0.26 & 35.6 & 18.2 \\
\hline Steph 430 & 11.22 & 1.53 & M3 & 15.4 & 366 & 0.058 & 0.016 & 20 & 16 & -0.50 & 0.21 & 32.8 & 9.3 \\
\hline HD 24409 & 6.54 & & G0 & 21.4 & 573 & 0.014 & 0.006 & 7 & 46 & -0.90 & 0.33 & 4.8 & 2.7 \\
\hline Gl 156.2 & 8.26 & 1.15 & $\mathrm{~K} 4 \mathrm{~V}$ & 16.0 & 480 & 0.026 & 0.010 & 12 & 16 & -0.68 & 0.20 & 12.4 & 3.8 \\
\hline *GJ 1066 & 8.94 & 1.21 & $\mathrm{~K} 4 \mathrm{~V}$ & 17.6 & 576 & 0.069 & 0.011 & 37 & 9 & -0.36 & 0.16 & 44.4 & 16.5 \\
\hline Gl $157 \mathrm{~A}$ & 8.04 & 1.11 & K4 V & 15.8 & 329 & 0.440 & 0.038 & 364 & 15 & -0.36 & 0.08 & 281.4 & 83.8 \\
\hline Gl 157 B & 11.61 & 1.47 & dM3 e & 15.8 & 329 & 0.440 & 0.038 & 364 & 15 & -0.36 & 0.08 & 281.4 & 83.8 \\
\hline
\end{tabular}




\begin{tabular}{|c|c|c|c|c|c|c|c|c|c|c|c|c|c|}
\hline Name & $V$ & $B-V$ & Sp. type & $d(\mathrm{pc})$ & Exp. & $C R$ & $\pm C R$ & $\mathrm{Li}$ & $\Delta$ & $h r$ & $\Delta h r$ & $f_{\mathrm{x}-14}$ & $L_{\mathrm{x} 27}$ \\
\hline Gl 159 & 5.37 & 0.50 & F6 V & 19.2 & 320 & 1.524 & 0.100 & 774 & 10 & -0.04 & 0.07 & 1234.1 & 546.1 \\
\hline Gl 160 & 5.90 & 0.62 & G5 V & 16.7 & 178 & 0.424 & 0.051 & 174 & 12 & -0.27 & 0.12 & 291.6 & 97.6 \\
\hline Rob 256 & 12.89 & & dM5 e & 16.3 & 327 & 0.155 & 0.024 & 103 & 23 & -0.22 & 0.15 & 110.4 & 34.9 \\
\hline Gl 161.1 & 5.52 & 0.46 & F7 V & 21.3 & 473 & 0.843 & 0.044 & 999 & 9 & -0.14 & 0.05 & 638.4 & 347.2 \\
\hline HD 26337 & 7.04 & & G2 IV-V & 56.2 & 308 & 4.409 & 0.286 & 760 & 11 & -0.15 & 0.06 & 3313.4 & 12511.7 \\
\hline G247-015 & 13.71 & 1.69 & $\mathrm{~m}$ & 11.8 & 478 & 0.052 & 0.012 & 33 & 6 & -0.52 & 0.18 & 29.0 & 4.8 \\
\hline HD 26018 & 8.19 & 0.84 & G5 & 26.4 & 469 & 0.065 & 0.014 & 33 & 5 & 0.03 & 0.21 & 55.0 & 45.9 \\
\hline Gl 165.1 & 8.67 & 1.00 & K3 & 27.9 & 523 & 0.049 & 0.012 & 31 & 11 & -0.46 & 0.19 & 28.8 & 26.9 \\
\hline Gl $166 \mathrm{~A}$ & 4.43 & 0.82 & $\mathrm{~K} 1 \mathrm{Ve}$ & 5.0 & 341 & 0.179 & 0.025 & 93 & 46 & -0.14 & 0.14 & 135.8 & 4.1 \\
\hline Gl $166 \mathrm{~B}$ & 9.52 & 0.03 & DA4 & 5.0 & 344 & 0.796 & 0.052 & 572 & 35 & -0.29 & 0.06 & 539.4 & 16.4 \\
\hline Gl $166 \mathrm{C}$ & 11.17 & 1.67 & $\mathrm{dM} 4.5 \mathrm{e}$ & 5.0 & 344 & 0.796 & 0.052 & 572 & 35 & -0.29 & 0.06 & 539.4 & 16.4 \\
\hline G007-034 & 13.82 & & $\mathrm{dM} 4 \mathrm{e}$ & 10.3 & 280 & 0.121 & 0.023 & 54 & 19 & -0.35 & 0.17 & 77.8 & 9.9 \\
\hline Gl 167 & 7.64 & 1.13 & K5 V & 13.1 & 233 & 0.035 & 0.015 & 8 & 24 & -1.00 & 9.99 & 10.6 & 2.2 \\
\hline Gl 167.1 & 4.25 & 0.30 & F4 III & 20.3 & 179 & 0.187 & 0.036 & 67 & 7 & -0.10 & 0.19 & 145.3 & 71.5 \\
\hline Gl 167.3 & 4.44 & 1.07 & K2 IV & 18.2 & 1131 & 0.013 & 0.005 & 12 & 19 & -1.00 & 0.32 & 3.8 & 1.5 \\
\hline LP $415-636$ & 12.90 & 1.70 & $\mathrm{dM} 4.5 \mathrm{e}$ & 22.6 & 462 & 0.108 & 0.017 & 77 & 23 & -0.28 & 0.14 & 73.9 & 45.2 \\
\hline *GJ 2034 & 14.36 & 0.52 & $\mathrm{DA} 8+\mathrm{M}$ & & 233 & 0.243 & 0.032 & 90 & 45 & -0.06 & 0.11 & 194.0 & \\
\hline LDS $3584 \mathrm{~A}$ & 12.63 & & $\mathrm{~m}$ & 23.5 & 410 & 0.043 & 0.012 & 24 & 38 & -0.27 & 0.26 & 29.2 & 19.4 \\
\hline LDS $3584 \mathrm{~B}$ & 14.97 & & $\mathrm{~m}$ & 23.5 & 410 & 0.043 & 0.012 & 24 & 35 & -0.27 & 0.26 & 29.2 & 19.4 \\
\hline Wo $9155 \mathrm{~A}$ & 6.88 & 0.66 & $\mathrm{G} 4 \mathrm{~V}$ & 27.1 & 544 & 0.051 & 0.012 & 23 & 26 & -0.89 & 0.20 & 18.5 & 16.2 \\
\hline Wo $9155 \mathrm{~B}$ & 7.30 & & G6 V & 27.1 & 544 & 0.051 & 0.012 & 23 & 17 & -0.89 & 0.20 & 18.5 & 16.2 \\
\hline LHS 1668 & 14.10 & & M3.5 & 17.0 & 727 & 0.062 & 0.012 & 58 & 64 & -0.07 & 0.17 & 49.5 & 17.1 \\
\hline G175-032 & 14.45 & & $\mathrm{~m}$ & 20.0 & 479 & 0.050 & 0.012 & 30 & 20 & -0.12 & 0.22 & 38.1 & 18.2 \\
\hline Gl 169 & 8.27 & 1.35 & $\mathrm{~K} 7 \mathrm{~V}$ & 11.5 & 429 & 0.133 & 0.020 & 116 & 6 & -0.37 & 0.13 & 84.6 & 13.3 \\
\hline Gl 170 & 13.91 & 1.73 & M4.5 & 10.4 & 465 & 0.087 & 0.015 & 64 & 24 & -0.30 & 0.16 & 58.3 & 7.6 \\
\hline LP $415-148$ & 14.57 & 1.75 & $\mathrm{k}-\mathrm{m}$ & 33.6 & 467 & 0.021 & 0.009 & 11 & 13 & -0.02 & 0.41 & 17.6 & 23.7 \\
\hline HD 28495 & 7.74 & & G0 & 27.5 & 476 & 0.528 & 0.034 & 653 & 15 & -0.18 & 0.06 & 388.8 & 353.0 \\
\hline G008-041 & 14.60 & & $\mathrm{~m}$ & 16.4 & 420 & 0.090 & 0.017 & 50 & 9 & -0.16 & 0.18 & 67.2 & 21.6 \\
\hline Wo 9158 & 7.93 & 0.79 & $\mathrm{~K} 1$ & 26.8 & 551 & 0.026 & 0.009 & 12 & 86 & 0.56 & 0.34 & 29.3 & 25.2 \\
\hline GJ 1071 & 8.86 & 1.11 & $\mathrm{~K} 5 \mathrm{~V}$ & 22.5 & 596 & 0.046 & 0.011 & 28 & 35 & -0.31 & 0.21 & 30.6 & 18.5 \\
\hline Gl $171.2 \mathrm{~A}$ & 8.42 & 1.12 & dK5 ep & 17.9 & 452 & 2.689 & 0.078 & 999 & 4 & -0.05 & 0.03 & 2163.2 & 825.3 \\
\hline Gl 172 & 8.61 & 1.40 & $\mathrm{~K} 8 \mathrm{Ve}$ & 10.2 & 512 & 0.079 & 0.014 & 63 & 5 & -0.64 & 0.12 & 39.0 & 4.8 \\
\hline G039-029 & 12.53 & 1.65 & $\mathrm{~m}$ & 9.8 & 452 & 0.310 & 0.028 & 324 & 5 & -0.20 & 0.09 & 224.6 & 25.9 \\
\hline Steph 497 & 10.59 & 1.45 & M1 & 15.1 & 509 & 1.594 & 0.155 & 275 & 8 & -0.09 & 0.10 & 1248.6 & 341.9 \\
\hline Steph 502 & 11.24 & 1.47 & M0 & 17.8 & 402 & 0.014 & 0.007 & 7 & 79 & 1.00 & 0.86 & 18.9 & 7.2 \\
\hline Wo $9163 \mathrm{AB}$ & 10.97 & 1.48 & dM0 & 20.2 & 471 & 0.016 & 0.007 & 8 & 37 & -1.00 & 0.51 & 4.9 & 2.4 \\
\hline Gl 174 & 8.00 & 1.10 & K3 V & 13.5 & 434 & 2.364 & 0.075 & 999 & 2 & -0.21 & 0.03 & 1701.4 & 370.7 \\
\hline Gl $174.1 \mathrm{~A}$ & 4.44 & 0.34 & $\mathrm{~F} 2 \mathrm{~V}$ & 20.1 & 329 & 0.521 & 0.042 & 393 & 7 & -0.33 & 0.08 & 341.6 & 165.4 \\
\hline Gl $174.1 \mathrm{~B}$ & 12.50 & & & 20.1 & 329 & 0.521 & 0.042 & 393 & 7 & -0.33 & 0.08 & 341.6 & 165.4 \\
\hline Gl 176 & 9.98 & 1.52 & $\mathrm{dM} 2.5 \mathrm{e}$ & 9.4 & 425 & 0.039 & 0.012 & 13 & 24 & -0.18 & 0.28 & 28.6 & 3.0 \\
\hline Gl 176.1 & 5.05 & 0.38 & $\mathrm{~F} 1 \mathrm{~V}$ & 27.6 & 268 & 0.099 & 0.022 & 39 & 11 & -0.16 & 0.20 & 73.7 & 67.3 \\
\hline Gl 176.3 & 7.59 & 0.89 & $\mathrm{~K} 0 \mathrm{~V}$ & 20.4 & 708 & 0.025 & 0.008 & 14 & 11 & -0.79 & 0.18 & 10.5 & 5.3 \\
\hline Gl 177 & 5.49 & 0.63 & G1 V & 13.3 & 539 & 0.490 & 0.046 & 272 & 9 & -0.34 & 0.09 & 319.0 & 67.7 \\
\hline Gl 178 & 3.19 & 0.45 & F6 V & $\begin{array}{r}10.0 \\
8.0\end{array}$ & 392 & 1.957 & 0.147 & 481 & 14 & -0.23 & 0.07 & 1387.7 & 106.9 \\
\hline GJ 2035 & 8.77 & 1.01 & $\mathrm{~K} 5 \mathrm{~V}$ & 27.0 & 442 & 0.065 & 0.014 & 36 & 5 & -0.09 & 0.22 & 50.8 & 44.1 \\
\hline GJ 2036 A & 11.13 & 1.57 & M2 Ve & 11.2 & 600 & 1.084 & 0.108 & 170 & 20 & -0.20 & 0.09 & 785.9 & 117.4 \\
\hline GJ $2036 \mathrm{~B}$ & 12.15 & 1.60 & & 11.2 & 600 & 1.084 & 0.108 & 170 & 20 & -0.20 & 0.09 & 785.9 & 117.4 \\
\hline Wo $9169 \mathrm{~A}$ & 7.02 & 0.55 & F8 & 36.0 & 406 & 0.149 & 0.022 & 106 & 30 & -0.27 & 0.14 & 102.8 & 159.1 \\
\hline Wo $9169 \mathrm{~B}$ & 14.60 & & $\mathrm{k}$ & 36.0 & 406 & 0.149 & 0.022 & 106 & 10 & -0.27 & 0.14 & 102.8 & 159.1 \\
\hline GJ 2037 & 8.13 & 1.07 & $\mathrm{~K} 3 / 4 \mathrm{~V}$ & 18.0 & 503 & 0.013 & 0.006 & 7 & 8 & -1.00 & 9.31 & 3.9 & 1.5 \\
\hline Gl 181 & 9.78 & 1.44 & $\mathrm{dM} 2 \mathrm{e}$ & 16.5 & 474 & 0.023 & 0.009 & 11 & 24 & -0.84 & 0.16 & 8.9 & 2.9 \\
\hline Gl 182 & 10.09 & 1.39 & $\mathrm{dM} 0.5$ & 26.7 & 393 & 0.651 & 0.042 & 667 & 14 & -0.20 & 0.06 & 472.3 & 401.8 \\
\hline Gl 183 & 6.22 & 1.06 & $\mathrm{~K} 3 \mathrm{~V}$ & 8.8 & 387 & 0.032 & 0.012 & 9 & 13 & -0.64 & 0.39 & 15.8 & 1.5 \\
\hline LP $476-207$ & 11.47 & 1.52 & dM3 & 32.1 & 420 & 0.661 & 0.060 & 340 & 6 & -0.35 & 0.08 & 427.0 & 524.8 \\
\hline LHS 1723 & 12.10 & & $\mathrm{~m}$ & 6.4 & 387 & 0.104 & 0.019 & 50 & 5 & -0.68 & 0.12 & 48.9 & 2.4 \\
\hline Gl $185 \mathrm{~A}$ & 8.46 & 1.41 & M0 V & 8.5 & 426 & 0.025 & 0.012 & 8 & 14 & -0.43 & 0.34 & 15.1 & 1.3 \\
\hline Gl $185 \mathrm{~B}$ & 10.50 & & & 8.5 & 426 & 0.025 & 0.012 & 8 & 14 & -0.43 & 0.34 & 15.1 & 1.3 \\
\hline G097-015 & 13.75 & & $\mathrm{~m}$ & 12.1 & 418 & 0.029 & 0.011 & 14 & 9 & -0.33 & 0.30 & 19.2 & 3.4 \\
\hline Gl 187 & 5.37 & 0.43 & $\mathrm{~F} 2 \mathrm{~V}$ & 26.2 & 244 & 0.179 & 0.030 & 60 & 8 & -0.23 & 0.16 & 126.9 & 104.1 \\
\hline Gl $187.2 \mathrm{~A}$ & 5.00 & 0.33 & $\mathrm{~F} 0 \mathrm{~V}$ & 26.2 & 450 & 0.051 & 0.013 & 22 & 19 & -0.83 & 0.16 & 19.8 & 16.3 \\
\hline LP $716-35$ & 12.97 & & $\mathrm{M} 4 \mathrm{e}$ & 17.4 & 381 & 0.045 & 0.013 & 28 & 6 & -0.36 & 0.24 & 28.7 & 10.4 \\
\hline HD 32850 & 7.75 & 0.80 & Ko V & 24.0 & 426 & 0.029 & 0.009 & 16 & 23 & -0.22 & 0.33 & 20.6 & 14.2 \\
\hline Gl 189 & 4.71 & 0.52 & F7 V & 11.7 & 1236 & 0.484 & 0.110 & 26 & 14 & -0.33 & 0.18 & 317.9 & 51.7 \\
\hline BD-21 1074 & 10.29 & 1.52 & M2 & 11.6 & 395 & 1.147 & 0.099 & 382 & 6 & -0.16 & 0.08 & 855.9 & 137.5 \\
\hline Steph 545 & 11.66 & 1.51 & M3: & 11.6 & 395 & 1.147 & 0.099 & 382 & 8 & -0.16 & 0.08 & 855.9 & 137.5 \\
\hline G085-041 & 11.78 & 1.66 & $\mathrm{k}$ & 12.8 & 445 & 0.055 & 0.014 & 24 & 5 & -0.58 & 0.22 & 28.9 & 5.7 \\
\hline GJ 1075 & 9.02 & 1.40 & $\mathrm{~K} 7 \mathrm{~V}$ & 11.5 & 1241 & 0.031 & 0.010 & 11 & 17 & -0.90 & 0.20 & 10.9 & 1.7 \\
\hline Wo 9175 & 2.79 & 0.13 & A3 III & 27.2 & 408 & 0.176 & 0.024 & 115 & 17 & -0.24 & 0.12 & 124.1 & 110.2 \\
\hline Gl 189.2 & 5.11 & 0.44 & $\mathrm{~F} 2 \mathrm{~V}$ & 25.0 & 406 & 0.021 & 0.009 & 8 & 50 & -1.00 & 0.42 & 6.4 & 4.8 \\
\hline G085-044 & 12.48 & 1.50 & M3 & 20.9 & 458 & 0.182 & 0.022 & 170 & 2 & -0.13 & $\begin{array}{l}0.42 \\
0.12\end{array}$ & $\begin{array}{r}0.4 \\
139.0\end{array}$ & $\begin{array}{r}4.0 \\
72.5\end{array}$ \\
\hline Gl 190 & 10.30 & 1.50 & M4 & 9.3 & 383 & 0.030 & 0.012 & 9 & 34 & -1.00 & 0.28 & 9.1 & 0.9 \\
\hline Gl 191 & 8.85 & 1.55 & Mo V & 3.9 & 248 & 0.049 & 0.020 & 10 & 43 & -0.75 & 0.15 & 21.1 & 0.4 \\
\hline Gl $194 \mathrm{~A}$ & 0.71 & 0.80 & G5 III & 12.9 & 420 & 23.540 & 0.244 & 999 & 15 & 0.11 & 0.01 & 20934.1 & 4191.6 \\
\hline Gl $194 \mathrm{~B}$ & 0.96 & & G0 III & 12.9 & 420 & 23.540 & 0.244 & 999 & 15 & 0.11 & 0.01 & 20934.1 & 4191.6 \\
\hline Gl $195 \mathrm{~A}$ & 10.20 & 1.50 & $\mathrm{dM} 2$ & 13.2 & 425 & 0.137 & 0.020 & 91 & 18 & -0.28 & 0.14 & 93.3 & 19.3 \\
\hline Gl $195 \mathrm{~B}$ & 13.70 & & M4 : & 13.2 & 425 & 0.137 & 0.020 & 91 & 18 & -0.28 & 0.14 & 93.3 & 19.3 \\
\hline Gl 196 & 5.05 & 0.47 & $\mathrm{~F} 6 \mathrm{~V}$ & 21.0 & 519 & 0.020 & 0.007 & 11 & 10 & -1.00 & 0.64 & 5.9 & 3.1 \\
\hline Gl 198 & 5.96 & 0.57 & G0 V & 24.9 & 417 & 0.028 & 0.010 & 14 & 48 & -0.04 & 0.37 & 22.8 & 16.9 \\
\hline Gl $200 \mathrm{~A}$ & 7.70 & 1.04 & $\mathrm{~K} 3 \mathrm{~V}$ & 16.9 & 412 & 0.030 & 0.010 & 11 & 57 & -0.88 & 0.22 & 11.0 & 3.7 \\
\hline Gl $200 \mathrm{~B}$ & 11.70 & & M2 & 16.9 & 412 & 0.030 & 0.010 & 11 & 57 & -0.88 & 0.22 & 11.0 & 3.7 \\
\hline
\end{tabular}




\begin{tabular}{|c|c|c|c|c|c|c|c|c|c|c|c|c|c|}
\hline Name & $V$ & $B-V$ & Sp. type & $d(\mathrm{pc})$ & Exp. & $C R$ & $\pm C R$ & $\mathrm{Li}$ & $\Delta$ & $h r$ & $\Delta h r$ & $f_{\mathrm{x}-14}$ & $L_{\mathrm{x} 27}$ \\
\hline Gl 199 A & 9.33 & 1.27 & $\mathrm{~K} 3 / 5 \mathrm{~V}$ & 20.3 & 399 & 0.134 & 0.020 & 72 & 4 & -0.36 & 0.13 & 85.7 & 42.2 \\
\hline Gl 199 B & 13.50 & & & 20.3 & 399 & 0.134 & 0.020 & 72 & 4 & -0.36 & 0.13 & 85.7 & 42.2 \\
\hline HD 34865 & 8.79 & 1.00 & $\mathrm{~K} 3 \mathrm{~V}$ & 24.3 & 431 & 0.023 & 0.009 & 10 & 8 & -0.36 & 0.39 & 14.7 & 10.4 \\
\hline Gl 201 & 7.95 & 1.09 & dK5 e & 14.3 & 444 & 0.110 & 0.017 & 92 & 10 & -0.49 & 0.13 & 62.8 & 15.4 \\
\hline Gl 202 & 4.99 & 0.53 & F8 Ve & 14.7 & 443 & 1.395 & 0.057 & 999 & 10 & -0.13 & 0.04 & 1063.1 & 273.5 \\
\hline GJ 1079 & 7.74 & 0.94 & $\mathrm{~K} 2 / 3 \mathrm{~V}$ & 17.9 & 519 & 0.022 & 0.009 & 8 & 20 & -0.58 & 0.28 & 11.6 & 4.4 \\
\hline G102-004 & 13.98 & 1.65 & $\mathrm{~m}$ & 29.0 & 454 & 0.024 & 0.009 & 15 & 9 & -0.35 & 0.30 & 15.2 & 15.3 \\
\hline Gl 204.1 & 6.99 & 0.77 & G5 Ve & 19.6 & 1150 & 0.106 & 0.017 & 71 & 18 & -0.52 & 0.12 & 59.2 & 27.1 \\
\hline Gl 205 & 7.96 & 1.47 & M1.5 V & 5.7 & 455 & 0.196 & 0.022 & 152 & 11 & -0.61 & 0.10 & 99.7 & 3.9 \\
\hline Gl 206 & 11.52 & 1.63 & $\mathrm{dM} 4 \mathrm{e}$ & 12.8 & 454 & 0.625 & 0.055 & 352 & 6 & -0.34 & 0.08 & 407.1 & 79.4 \\
\hline GJ 1081 & 12.21 & 1.60 & $\mathrm{k}-\mathrm{m}$ & 15.3 & 403 & 0.086 & 0.016 & 48 & 16 & -0.25 & 0.18 & 60.1 & 16.9 \\
\hline Gl 207.1 & 11.53 & 1.57 & $\mathrm{dM} 2.5 \mathrm{e}$ & 16.8 & 459 & 0.505 & 0.050 & 286 & 5 & -0.18 & 0.10 & 371.8 & 125.6 \\
\hline G097-054 & 11.81 & 1.59 & M3.5 & 12.4 & 453 & 0.022 & 0.009 & 10 & 32 & -0.02 & 0.39 & 18.2 & 3.4 \\
\hline Gl 208 & 8.80 & 1.40 & dMO & 11.4 & 463 & 0.274 & 0.026 & 259 & 16 & -0.46 & 0.09 & 160.7 & 24.9 \\
\hline Wo 9185 & 9.33 & 1.13 & K5 V & 32.5 & 1692 & 0.016 & 0.004 & 16 & 45 & -0.32 & 0.26 & 10.6 & 13.3 \\
\hline Gl 209.1 & 5.30 & 0.46 & F5 V & 42.6 & 526 & 0.125 & 0.017 & 109 & 12 & -0.14 & 0.14 & 94.3 & 204.3 \\
\hline Gl 211 & 6.23 & 0.84 & $\mathrm{~K} 1 \mathrm{Ve}$ & 12.2 & 367 & 0.369 & 0.033 & 331 & 14 & -0.55 & 0.08 & 199.0 & 35.7 \\
\hline GJ 1083 AB & 14.85 & 1.88 & $\mathrm{~m}$ & 10.4 & 435 & 0.043 & 0.012 & 22 & 28 & 0.01 & 0.25 & 35.8 & 4.6 \\
\hline Gl 212 & 9.75 & 1.48 & M1 & 12.5 & 367 & 0.369 & 0.033 & 331 & 80 & -0.55 & 0.08 & 199.0 & 37.1 \\
\hline G102-021 & 11.36 & 1.46 & M3 & 28.8 & 462 & 0.319 & 0.028 & 350 & 9 & -0.27 & 0.08 & 219.2 & 217.8 \\
\hline Wo 9188 & 10.61 & 1.46 & dMo & 21.5 & 448 & 0.017 & 0.008 & 9 & 6 & 0.58 & 0.52 & 19.7 & 10.9 \\
\hline Gl 213 & 11.53 & 1.62 & M4 & 5.8 & 461 & 0.016 & 0.007 & 9 & 14 & -0.61 & 0.39 & 8.2 & 0.3 \\
\hline Wo 9189 & 5.65 & 0.60 & $\mathrm{G} 1 \mathrm{~V}$ & 18.2 & 1069 & 0.016 & 0.005 & 12 & 10 & -0.72 & 0.21 & 7.0 & 2.8 \\
\hline Gl $216 \mathrm{~B}$ & 6.13 & 0.94 & $\mathrm{~K} 2 \mathrm{~V}$ & 9.0 & 488 & 0.343 & 0.029 & 330 & 10 & -0.52 & 0.06 & 190.5 & 18.3 \\
\hline Steph 578 & 10.99 & 1.45 & M0 & 24.4 & 478 & 0.205 & 0.022 & 193 & 7 & -0.28 & 0.11 & 140.2 & 100.3 \\
\hline GJ 1084 & 9.74 & 1.38 & Mo V & 20.7 & 916 & 0.037 & 0.008 & 47 & 8 & -0.49 & 0.16 & 21.3 & 11.0 \\
\hline GJ 1085 & 5.97 & 0.64 & $\mathrm{G} 2 \mathrm{~V}$ & 15.6 & 500 & 0.023 & 0.008 & 10 & 41 & -0.78 & 0.23 & 9.5 & 2.7 \\
\hline Gl $222 \mathrm{AB}$ & 4.40 & 0.59 & G0 V & 8.7 & 431 & 1.942 & 0.068 & 999 & 9 & -0.27 & 0.03 & 1335.9 & 120.0 \\
\hline Gl 224.1 & 4.65 & 1.05 & K1 III/IV & 27.2 & 3067 & 0.007 & 0.002 & 11 & 31 & -1.00 & 0.73 & 2.1 & 1.9 \\
\hline Gl 225 & 3.72 & 0.33 & F1 III & 15.0 & 507 & 0.224 & 0.023 & 212 & 12 & -0.37 & 0.09 & 142.0 & 38.4 \\
\hline G192-012 & 13.56 & & $\mathrm{~m}$ & 13.5 & 343 & 0.140 & 0.023 & 75 & 9 & -0.34 & 0.15 & 91.0 & 19.8 \\
\hline G099-049 & 11.33 & 1.68 & M4 & 5.4 & 475 & 0.404 & 0.045 & 193 & 7 & -0.20 & 0.11 & 292.9 & 10.1 \\
\hline Gl 226 & 10.50 & 1.51 & M2.5 & 9.4 & 534 & 0.019 & 0.008 & 7 & 20 & -0.81 & 0.20 & 7.8 & 0.8 \\
\hline Wo 9200 & 6.35 & 0.52 & G0 IV-V & 26.7 & 933 & 0.636 & 0.028 & 889 & 77 & -0.09 & 0.05 & 498.1 & 423.8 \\
\hline Gl 227 & 6.76 & 0.81 & dKo e & 15.5 & 434 & 0.257 & 0.027 & 200 & 13 & -0.39 & 0.10 & 160.8 & 45.9 \\
\hline Gl 229 & 8.14 & 1.50 & M1 Ve & 5.8 & 592 & 0.054 & 0.012 & 39 & 7 & -0.38 & 0.18 & 33.7 & 1.3 \\
\hline Gl 230 & 6.45 & 0.67 & $\mathrm{G} 2 \mathrm{~V}$ & 18.1 & 450 & 0.193 & 0.023 & 172 & 5 & -0.37 & 0.10 & 122.6 & 48.1 \\
\hline HD 43162 & 6.38 & 0.72 & G5 V & 16.7 & 651 & 0.655 & 0.071 & 165 & 21 & -0.39 & 0.10 & 408.7 & 136.3 \\
\hline Gl 231 & 5.08 & 0.72 & G5 V V & 10.2 & 1166 & 0.017 & 0.005 & 21 & 16 & -1.00 & 1.53 & 5.1 & 0.6 \\
\hline HD 43042 & 5.20 & 0.44 & F6 V & 21.1 & 403 & 0.140 & 0.020 & 95 & 8 & -0.18 & 0.14 & 102.8 & 55.0 \\
\hline Wo 9207 & 5.04 & 0.42 & F5 IV-V & 19.6 & 454 & 0.352 & 0.029 & 380 & 1 & -0.24 & 0.08 & 247.7 & 113.9 \\
\hline G101-035 & 12.27 & & $\mathrm{~m}$ & 23.3 & 299 & 0.265 & 0.033 & 156 & 7 & -0.30 & 0.12 & 178.0 & 115.7 \\
\hline Gl $233 \mathrm{AB}$ & 6.76 & 0.94 & $\mathrm{~K} 2 \mathrm{~V}$ e & 14.7 & 394 & 1.741 & 0.069 & 999 & 6 & -0.20 & 0.04 & 1262.2 & 324.7 \\
\hline Steph 598 & 11.09 & 1.50 & M0 & 19.3 & 285 & 0.360 & 0.037 & 214 & 17 & -0.32 & 0.10 & 238.2 & 106.6 \\
\hline LP 205-49 & 14.83 & & $\mathrm{~m}$ & 12.5 & 311 & 0.085 & 0.019 & 42 & 4 & -0.22 & 0.21 & 60.7 & 11.4 \\
\hline Gl 239 & 9.63 & 1.49 & $\mathrm{dM} 1$ & 9.8 & 447 & 0.025 & 0.009 & 15 & 13 & -0.33 & 0.35 & 16.7 & 1.9 \\
\hline Gl 240 & 9.62 & 1.46 & Ko & 15.1 & 948 & 0.041 & 0.009 & 39 & 3 & -0.61 & 0.14 & 20.7 & 5.7 \\
\hline HD 48189 A & 6.34 & 0.62 & G0 V V & 21.6 & 966 & 2.325 & 0.050 & 999 & 6 & -0.15 & 0.02 & 1747.2 & 979.4 \\
\hline HD $48189 \mathrm{~B}$ & 8.30 & & & 21.6 & 966 & 2.325 & 0.050 & 999 & 6 & -0.15 & 0.02 & 1747.2 & 979.4 \\
\hline Gl 240.1 & 5.45 & 0.50 & F8 V & 17.9 & 528 & 0.017 & 0.007 & 9 & 20 & -1.00 & 0.89 & 5.0 & 1.9 \\
\hline Gl 241 & 8.13 & 1.02 & dK 6 & 17.5 & 439 & 0.023 & 0.010 & 7 & 9 & -0.04 & 0.37 & 18.9 & 7.0 \\
\hline Wo 9216 & 10.95 & 1.48 & K7 & 22.5 & 411 & 0.021 & 0.009 & 10 & 5 & -1.00 & 0.38 & 6.4 & 3.9 \\
\hline G192-039 & 12.44 & 1.59 & M3.5 & 19.1 & 298 & 0.051 & 0.016 & 14 & 15 & -0.41 & 0.30 & 31.1 & 13.6 \\
\hline G192-039 & 14.90 & & & 19.1 & 298 & 0.051 & 0.016 & 14 & 15 & -0.41 & 0.30 & 31.1 & 13.6 \\
\hline Gl 242 & 3.36 & 0.43 & F5 III & 17.5 & 451 & 0.393 & 0.031 & 443 & 6 & -0.04 & 0.08 & 318.6 & 117.3 \\
\hline Gl $244 \mathrm{~A}$ & -1.43 & & $\mathrm{~A} 1 \mathrm{~V}$ & 2.6 & 272 & 12.730 & 0.219 & 999 & 8 & -1.00 & 0.00 & 3831.7 & 31.9 \\
\hline Gl 244 B & 8.44 & -0.03 & DA2 & 2.6 & 272 & 12.730 & 0.219 & 999 & 8 & -1.00 & 0.00 & 3831.7 & 31.9 \\
\hline Gl 245 & 5.24 & 0.56 & Go V & 16.5 & 337 & 0.048 & 0.014 & 17 & 43 & -0.19 & 0.30 & 34.8 & 11.3 \\
\hline Gl 245.1 & 5.92 & 0.49 & F6 V & 24.3 & 577 & 0.015 & 0.006 & 9 & 24 & -0.65 & 0.32 & 7.1 & 5.0 \\
\hline Gl 248 & 3.27 & 0.21 & A7 IV & 30.3 & 1213 & 0.078 & 0.009 & 138 & 6 & -0.13 & 0.12 & 59.2 & 65.1 \\
\hline Gl 249.1 & 5.13 & 0.45 & F5 III & 25.2 & 606 & 0.016 & 0.006 & 10 & 30 & -0.88 & 0.32 & 5.8 & 4.4 \\
\hline Gl $250 \mathrm{~A}$ & 6.59 & 1.05 & K3 V & 8.7 & 269 & 0.229 & 0.032 & 152 & 7 & -0.51 & 0.12 & 128.6 & 11.7 \\
\hline Gl $250 \mathrm{~B}$ & 10.09 & 1.50 & M2 & 8.7 & 269 & 0.229 & 0.032 & 152 & 66 & -0.51 & 0.12 & 128.6 & 11.7 \\
\hline Wo $9220 \mathrm{~A}$ & 4.74 & 0.31 & A9 V p & 27.9 & 344 & 0.263 & 0.031 & 196 & 5 & -0.22 & 0.11 & 188.2 & 175.7 \\
\hline Wo $9220 \mathrm{~B}$ & 7.68 & 0.72 & G6 V & 27.9 & 344 & 0.263 & 0.031 & 196 & 5 & -0.22 & 0.11 & 188.2 & 175.7 \\
\hline G250-031 & 13.65 & & $\mathrm{~m}$ & 11.4 & 350 & 0.331 & 0.033 & 242 & 8 & 0.01 & 0.10 & 277.2 & 43.4 \\
\hline Wo 9222 & 7.65 & 0.53 & $\mathrm{~F} 8 \mathrm{~V}+\mathrm{A} / \mathrm{F}$ & 53.8 & 804 & 0.018 & 0.006 & 17 & 25 & & & 15.0 & 52.0 \\
\hline Gl $255 \mathrm{~A}$ & 6.91 & 0.46 & F8 IV-V & 43.3 & 432 & 0.412 & 0.032 & 462 & 6 & -0.02 & 0.08 & 338.0 & 757.9 \\
\hline Gl $255 \mathrm{~B}$ & 7.10 & & F8 IV-V & 43.3 & 432 & 0.412 & 0.032 & 462 & 6 & -0.02 & 0.08 & 338.0 & 757.9 \\
\hline Gl 256 & 9.15 & 1.16 & $\mathrm{~K} 4 \mathrm{~V}$ & 22.3 & 445 & 0.503 & 0.034 & 661 & 6 & -0.24 & 0.07 & 354.2 & 211.2 \\
\hline Gl 259 & 6.71 & 0.89 & $\mathrm{KO} \mathrm{Ve}$ & 14.6 & 443 & 0.198 & 0.024 & 154 & 4 & -0.42 & 0.10 & 120.8 & 30.9 \\
\hline Gl 260 & 6.82 & 0.80 & K0 IV-V & 18.4 & 1481 & 0.192 & 0.012 & 557 & 8 & -0.38 & 0.06 & 120.7 & 49.0 \\
\hline GJ 1094 & 8.38 & 1.08 & K5 V & 18.7 & 416 & 0.022 & 0.009 & 8 & 35 & -0.52 & 0.34 & 12.2 & 5.1 \\
\hline Gl 268 & 11.49 & 1.71 & $\mathrm{M} 4.5 \mathrm{Ve}$ & 6.4 & 202 & 0.257 & 0.041 & 79 & 20 & -0.56 & 0.12 & 137.3 & 6.6 \\
\hline Gl 268.1 & 4.48 & 0.32 & Fo IV & 21.2 & 176 & 0.106 & 0.027 & 34 & 22 & -0.58 & 0.19 & 55.6 & 29.9 \\
\hline Gl 268.2 & 9.10 & 1.25 & K5 V & 20.0 & 1217 & 0.013 & 0.005 & 10 & 17 & -0.65 & 0.22 & 6.4 & 3.1 \\
\hline GJ 1096 & 14.48 & 1.75 & $\mathrm{~m}+$ & 14.9 & 437 & 0.081 & 0.015 & 56 & 15 & -0.39 & 0.17 & 50.4 & 13.5 \\
\hline Gl 268.3 & 10.85 & 1.54 & dMO & 12.3 & 402 & 0.020 & 0.009 & 9 & 83 & -1.00 & 0.46 & 6.1 & 1.1 \\
\hline Gl 269 A & 7.15 & 0.99 & $\mathrm{~K} 1 \mathrm{~V}$ & 14.8 & 175 & 0.251 & 0.041 & 101 & 6 & -0.06 & 0.16 & 200.7 & 52.4 \\
\hline Gl 269 B & 7.90 & & $\mathrm{~K} 4 \mathrm{~V}$ & 14.8 & 175 & 0.251 & 0.041 & 101 & 6 & -0.06 & 0.16 & 200.7 & 52.4 \\
\hline
\end{tabular}




\begin{tabular}{|c|c|c|c|c|c|c|c|c|c|c|c|c|c|}
\hline Name & $V$ & $B-V$ & Sp. type & $d(\mathrm{pc})$ & Exp. & $C R$ & $\pm C R$ & $\mathrm{Li}$ & $\Delta$ & $h r$ & $\Delta h r$ & $f_{\mathrm{x}-14}$ & $L_{\mathrm{x} 27}$ \\
\hline Gl $271 \mathrm{~A}$ & 3.53 & 0.34 & F1 IV-V & 18.0 & 443 & 0.108 & 0.019 & 57 & 18 & -0.57 & 0.13 & 57.2 & 22.2 \\
\hline Gl $271 \mathrm{~B}$ & 8.20 & & K3 V & 18.0 & 443 & 0.108 & 0.019 & 57 & 18 & -0.57 & 0.13 & 57.2 & 22.2 \\
\hline Gl 272 & 10.53 & 1.46 & dM2 & 16.2 & 412 & 0.015 & 0.007 & 11 & 15 & -0.35 & 0.40 & 9.7 & 3.1 \\
\hline LHS 1909 & 11.75 & & $\mathrm{~m}$ & 22.4 & 388 & 0.028 & 0.010 & 18 & 39 & -0.31 & 0.31 & 18.4 & 11.1 \\
\hline Gl 273 & 9.85 & 1.56 & M3.5 & 3.8 & 247 & 0.034 & 0.015 & 9 & 39 & -1.00 & 0.74 & 10.2 & 0.2 \\
\hline Gl 273.1 & 7.74 & 0.94 & $\mathrm{dK} 8$ & 17.5 & 441 & 0.055 & 0.013 & 31 & 19 & -0.62 & 0.18 & 27.7 & 10.2 \\
\hline Gl $274 \mathrm{~A}$ & 4.18 & 0.32 & F0 V & 18.5 & 445 & 0.347 & 0.029 & 399 & 1 & -0.29 & 0.08 & 234.9 & 96.0 \\
\hline Gl 274 B & 12.50 & & & 18.5 & 445 & 0.347 & 0.029 & 399 & 1 & -0.29 & 0.08 & 234.9 & 96.0 \\
\hline Gl 277 A & 10.58 & 1.47 & dM3.5e & 11.4 & 455 & 1.006 & 0.048 & 999 & 30 & -0.21 & 0.05 & 724.0 & 112.9 \\
\hline Gl 277 B & 11.78 & 1.52 & $\mathrm{dM} 4.5 \mathrm{e}$ & 11.5 & 455 & 1.006 & 0.048 & 999 & 8 & -0.21 & 0.05 & 724.0 & 113.9 \\
\hline HD 59967 & 6.64 & 0.63 & G4 V & 21.8 & 271 & 0.320 & 0.037 & 195 & 13 & -0.36 & 0.10 & 205.1 & 116.5 \\
\hline Gl 278 A & 1.94 & 0.04 & $\mathrm{~A} 1 \mathrm{~V}$ & 15.8 & 440 & 3.697 & 0.091 & 999 & 58 & -0.16 & 0.03 & 2758.7 & 823.7 \\
\hline Gl $278 \mathrm{~B}$ & 2.85 & & A m & 15.8 & 440 & 3.697 & 0.091 & 999 & 58 & -0.16 & 0.03 & 2758.7 & 823.7 \\
\hline Gl $278 \mathrm{C}$ & 9.07 & 1.49 & $\mathrm{M} 0.5 \mathrm{Ve}$ & 15.8 & 440 & 3.697 & 0.091 & 999 & 16 & -0.16 & 0.03 & 2758.7 & 823.7 \\
\hline Gl $280 \mathrm{~A}$ & 0.38 & 0.42 & F5 IV-V & 3.5 & 400 & 3.655 & 0.098 & 999 & 8 & -0.90 & 0.01 & 1293.9 & 18.9 \\
\hline Gl $280 \mathrm{~B}$ & 10.70 & & DA & 3.5 & 400 & 3.655 & 0.098 & 999 & 8 & -0.90 & 0.01 & 1293.9 & 18.9 \\
\hline Gl $282 \mathrm{~A}$ & 7.20 & 0.96 & $\mathrm{~K} 2 \mathrm{Ve}$ & 14.2 & 128 & 0.128 & 0.038 & 18 & 13 & -0.67 & 0.20 & 60.9 & 14.7 \\
\hline Gl 282 B & 8.94 & 1.33 & K5 & 14.2 & 128 & 0.128 & 0.038 & 18 & 49 & -0.67 & 0.20 & 60.9 & 14.7 \\
\hline Gl 285 & 11.20 & 1.60 & $\mathrm{dM} 4.5 \mathrm{e}$ & 5.9 & 253 & 1.467 & 0.079 & 999 & 30 & -0.21 & 0.05 & 1055.8 & 44.4 \\
\hline Gl 285.1 & 7.09 & 0.66 & dG5 & 28.5 & 307 & 0.089 & 0.019 & 41 & 3 & -0.65 & 0.24 & 43.2 & 42.0 \\
\hline Gl 286 & 1.14 & 1.00 & K0 IIIb & 10.3 & 411 & 0.059 & 0.014 & 23 & 12 & -0.75 & 0.19 & 25.7 & 3.3 \\
\hline GJ 1101 & 13.09 & 1.68 & $\mathrm{~m}$ & 12.5 & 680 & 0.183 & 0.018 & 239 & 3 & -0.22 & 0.09 & 130.4 & 24.2 \\
\hline Gl 290 & 6.56 & 0.73 & G8 V & 17.0 & 551 & 0.021 & 0.008 & 9 & 23 & -0.67 & 0.26 & 9.8 & 3.4 \\
\hline Gl $292 \mathrm{~A}$ & 5.05 & 0.44 & F5 V & 18.0 & 154 & 0.589 & 0.063 & 226 & 9 & -0.20 & 0.11 & 426.9 & 165.8 \\
\hline Gl 292 B & 8.60 & & K3 & 18.0 & 154 & 0.589 & 0.063 & 226 & 9 & -0.20 & 0.11 & 426.9 & 165.8 \\
\hline LHS 1955 & 13.38 & & M4 & 12.6 & 243 & 0.037 & 0.016 & 8 & 45 & -0.35 & 0.35 & 23.8 & 4.5 \\
\hline Gl $293.1 \mathrm{~A}$ & 8.06 & 1.04 & K5 V & 17.5 & 366 & 0.024 & 0.010 & 8 & 23 & -0.58 & 0.32 & 12.5 & 4.5 \\
\hline Gl 293.1B & 13.30 & & & 17.5 & 366 & 0.024 & 0.010 & 8 & 23 & -0.58 & 0.32 & 12.5 & 4.5 \\
\hline Gl 293.2 & 8.42 & 1.05 & K3 V & 18.2 & 221 & 0.036 & 0.015 & 13 & 21 & -0.75 & 0.27 & 15.8 & 6.3 \\
\hline Wo 9247 & 7.95 & 0.74 & G6 V & 29.3 & 220 & 0.029 & 0.014 & 8 & 22 & -0.88 & 0.81 & 10.5 & 10.8 \\
\hline HD 67199 & 7.14 & 0.94 & $\mathrm{~K} 1 \mathrm{~V}$ & 17.3 & 929 & 0.036 & 0.008 & 31 & 35 & -0.48 & 0.16 & 20.8 & 7.4 \\
\hline $\mathrm{BD}+261715$ & 10.21 & 1.46 & M0 & 17.9 & 403 & 0.021 & 0.009 & 9 & 9 & -0.74 & 0.24 & 9.0 & 3.4 \\
\hline G040-007 & 9.80 & 1.38 & K5 & 17.1 & 212 & 0.767 & 0.064 & 458 & 15 & -0.31 & 0.08 & 511.4 & 179.4 \\
\hline LHS 5134 & 11.00 & & $\mathrm{~m}$ & 17.1 & 212 & 0.767 & 0.064 & 458 & 16 & -0.31 & 0.08 & 511.4 & 179.4 \\
\hline GJ 1108 A & 10.05 & 1.35 & $\mathrm{dM} 0.5 \mathrm{e}$ & 20.7 & 274 & 0.777 & 0.056 & 504 & 12 & -0.29 & 0.07 & 526.4 & 270.0 \\
\hline GJ 1108 B & 12.12 & 1.53 & dM3 e & 20.7 & 274 & 0.777 & 0.056 & 504 & 3 & -0.29 & 0.07 & 526.4 & 270.0 \\
\hline Gl 297.1 & 4.76 & 0.43 & F5 V & 21.4 & 366 & 0.351 & 0.032 & 292 & 5 & -0.19 & 0.09 & 256.6 & 140.2 \\
\hline Gl 302 & 5.97 & 0.76 & G7.5 V & 12.6 & 355 & 0.032 & 0.011 & 13 & 6 & -0.65 & 0.29 & 15.7 & 3.0 \\
\hline Gl 305 & 4.07 & 0.40 & F6 IV & 19.5 & 560 & 0.229 & 0.022 & 257 & 6 & -0.13 & 0.09 & 174.9 & 79.2 \\
\hline Gl 306 & 5.61 & 0.46 & F3 V & 27.2 & 307 & 0.686 & 0.050 & 548 & 16 & 0.01 & 0.07 & 574.0 & 507.1 \\
\hline GJ 2069 A & 11.89 & & M5 e & 12.8 & 314 & 0.726 & 0.050 & 580 & 5 & -0.14 & 0.07 & 549.5 & 107.8 \\
\hline GJ 2069 B & 13.32 & & & 12.8 & 314 & 0.726 & 0.050 & 580 & 17 & -0.14 & 0.07 & 549.5 & 107.8 \\
\hline LHS 2024 & 15.00 & & $\mathrm{~m}$ & 16.5 & 245 & 0.016 & 0.010 & 9 & 63 & & & 13.4 & 4.4 \\
\hline G234-037 & 11.67 & 1.57 & M3 & 12.9 & 430 & 0.037 & 0.011 & 15 & 25 & -1.00 & 0.41 & 11.1 & 2.2 \\
\hline HD 72760 & 7.32 & & G5 & 21.7 & 294 & 0.096 & 0.022 & 39 & 8 & -0.34 & 0.19 & 62.8 & 35.5 \\
\hline Gl $310.1 \mathrm{~A}$ & 5.99 & 0.52 & F8 V & 26.5 & 333 & 0.118 & 0.021 & 49 & 8 & -0.52 & 0.14 & 65.8 & 55.4 \\
\hline Gl 310.1B & 7.25 & 0.71 & G5 IV-V & 23.4 & 333 & 0.118 & 0.021 & 49 & 14 & -0.52 & 0.14 & 65.8 & 43.2 \\
\hline Gl 311 & 5.64 & 0.62 & G1 V & 14.3 & 392 & 0.797 & 0.047 & 820 & 6 & -0.32 & 0.06 & 526.9 & 128.3 \\
\hline Wo 9273 & 6.75 & 0.66 & G0 & 23.6 & 282 & 0.130 & 0.024 & 57 & 4 & -0.26 & 0.17 & 90.2 & 60.3 \\
\hline Gl 314 A & 5.28 & 0.73 & G3 V & 19.9 & 374 & 0.047 & 0.014 & 17 & 22 & -0.76 & 0.21 & 20.1 & 9.6 \\
\hline Gl 314 B & 6.80 & & KO V & 19.9 & 374 & 0.047 & 0.014 & 17 & 22 & -0.76 & 0.21 & 20.1 & 9.6 \\
\hline Gl 316.1 & 17.68 & 2.02 & $\mathrm{~m}$ & 14.1 & 170 & 0.417 & 0.054 & 127 & 20 & -0.07 & 0.13 & 331.1 & 78.4 \\
\hline Gl 320 & 6.56 & 0.93 & K1 V & 11.1 & 505 & 0.460 & 0.047 & 216 & 14 & -0.31 & 0.10 & 306.9 & 45.5 \\
\hline Gl 321.3A & 2.02 & 0.04 & A0 V & 24.4 & 553 & 0.264 & 0.034 & 109 & 11 & -0.24 & 0.13 & 185.9 & 133.0 \\
\hline Gl 321.3B & 5.00 & & & 24.4 & 553 & 0.264 & 0.034 & 109 & 11 & -0.24 & 0.13 & 185.9 & 133.0 \\
\hline Gl 321.3C & 11.00 & & & 24.4 & 553 & 0.264 & 0.034 & 109 & 60 & -0.24 & 0.13 & 185.9 & 133.0 \\
\hline Gl 321.3D & 13.50 & & & 24.4 & 553 & 0.264 & 0.034 & 109 & 60 & -0.24 & 0.13 & 185.9 & 133.0 \\
\hline Gl 322 & 9.28 & 1.34 & dM0 & 16.5 & 421 & 0.075 & 0.016 & 32 & 26 & -0.23 & 0.19 & 53.0 & 17.3 \\
\hline Gl 323 A & 9.77 & 1.36 & $\mathrm{dM} 0 \mathrm{p}$ & 17.0 & 246 & 0.097 & 0.024 & 26 & 17 & 0.04 & 0.24 & 82.9 & 28.6 \\
\hline Gl 323 B & 9.90 & & & 17.0 & 246 & 0.097 & 0.024 & 26 & 17 & 0.04 & 0.24 & 82.9 & 28.6 \\
\hline Gl $325 \mathrm{~A}$ & 8.70 & 1.39 & K5 V & 11.4 & 423 & 0.059 & 0.014 & 25 & 19 & -1.00 & 0.11 & 17.8 & 2.8 \\
\hline Gl 325 B & 8.90 & & K6 V & 11.4 & 423 & 0.059 & 0.014 & 25 & 18 & -1.00 & 0.11 & 17.8 & 2.8 \\
\hline Gl 327 & 6.00 & 0.67 & G3 V & 17.1 & 307 & 0.118 & 0.023 & 49 & 11 & -0.58 & 0.14 & 61.8 & 21.6 \\
\hline Gl 329 & 8.66 & 1.00 & K2/3 III & 31.2 & 232 & 0.030 & 0.013 & 9 & 37 & & & 24.8 & 28.8 \\
\hline LHS 2071 & 14.00 & & $\mathrm{~m}$ & 12.7 & 242 & 0.036 & 0.015 & 10 & 26 & -0.27 & 0.37 & 24.5 & 4.8 \\
\hline HD 76653 & 5.71 & 0.48 & F6 V & 24.2 & 440 & 0.461 & 0.034 & 403 & 2 & -0.31 & 0.07 & 307.0 & 214.3 \\
\hline G252-025 & 14.32 & 1.70 & $\mathrm{~m}$ & 13.8 & 423 & 0.018 & 0.008 & 8 & 65 & 0.34 & 0.46 & 18.6 & 4.2 \\
\hline GJ 1116 A & 14.06 & 1.84 & $\mathrm{~m}$ & 5.2 & 337 & 0.340 & 0.034 & 231 & 66 & -0.17 & 0.10 & 251.9 & 8.2 \\
\hline GJ 1116 B & 14.92 & 1.93 & $\mathrm{~m}$ & 5.2 & 337 & 0.340 & 0.034 & 231 & 60 & -0.17 & 0.10 & 251.9 & 8.2 \\
\hline Gl 331 A & 3.14 & 0.19 & A7 IV & 14.6 & 285 & 0.169 & 0.027 & 81 & 6 & -0.35 & 0.14 & 109.3 & 28.0 \\
\hline Gl 331 B & 10.80 & & dM1 & 14.6 & 285 & 0.169 & 0.027 & 81 & 6 & -0.35 & 0.14 & 109.3 & 28.0 \\
\hline Gl $331 \mathrm{C}$ & 11.00 & & & 14.6 & 285 & 0.169 & 0.027 & 81 & 6 & -0.35 & 0.14 & 109.3 & 28.0 \\
\hline G041-014 & 10.89 & 1.67 & $\mathrm{k}$ & 6.5 & 351 & 1.526 & 0.119 & 441 & 8 & -0.13 & 0.08 & 1163.0 & 58.1 \\
\hline GJ 1119 & 13.32 & 1.72 & $\mathrm{~m}$ & 10.3 & 308 & 0.031 & 0.013 & 10 & 26 & -0.15 & 0.37 & 23.2 & 3.0 \\
\hline Gl 332 A & 4.11 & 0.37 & F3 V & 16.4 & 400 & 0.676 & 0.045 & 543 & 6 & -0.46 & 0.06 & 396.9 & 128.1 \\
\hline Gl 332 B & 6.18 & 0.65 & G5 V & 16.4 & 400 & 0.676 & 0.045 & 543 & 6 & -0.46 & 0.06 & 396.9 & 128.1 \\
\hline HD 77137 & 6.87 & 0.69 & G5 V & 55.9 & 424 & 1.604 & 0.127 & 492 & 11 & 0.15 & 0.08 & 1460.4 & 5453.4 \\
\hline Gl 333.1 & 5.16 & 0.42 & F3 V & 26.2 & 355 & 0.219 & 0.028 & 155 & 8 & -0.32 & 0.11 & 144.6 & 118.6 \\
\hline Gl 334 & 9.51 & 1.42 & dM0 & 14.5 & 362 & 0.073 & 0.017 & 36 & 5 & -0.26 & 0.21 & 50.5 & 12.7 \\
\hline Gl 334.2 & 5.93 & 0.60 & F9 V & 19.1 & 411 & 0.334 & 0.030 & 284 & 8 & -0.42 & 0.08 & 203.1 & 88.9 \\
\hline
\end{tabular}




\begin{tabular}{|c|c|c|c|c|c|c|c|c|c|c|c|c|c|}
\hline Name & $V$ & $B-V$ & Sp. type & $d(\mathrm{pc})$ & Exp. & $C R$ & $\pm C R$ & $\mathrm{Li}$ & $\Delta$ & $h r$ & $\Delta h r$ & $f_{\mathrm{x}-14}$ & $L_{\mathrm{x} 27}$ \\
\hline Wo 9286 & 5.99 & 0.65 & G5 IV & 31.2 & 365 & 0.090 & 0.018 & 39 & 43 & -0.65 & 0.14 & 43.7 & 51.1 \\
\hline Gl $335 \mathrm{~A}$ & 4.84 & 0.49 & F7 IV-V & 20.4 & 438 & 0.059 & 0.014 & 23 & 24 & -0.79 & 0.16 & 24.2 & 12.1 \\
\hline Gl 335 B & 8.44 & & $\mathrm{~K} 2 \mathrm{~V}$ & 20.4 & 438 & 0.059 & 0.014 & 23 & 24 & -0.79 & 0.16 & 24.2 & 12.1 \\
\hline Wo $9287 \mathrm{~A}$ & 6.77 & 0.57 & G1 V & 44.6 & 439 & 0.028 & 0.010 & 10 & 85 & -0.61 & 0.35 & 14.4 & 34.4 \\
\hline Wo 9287 B & 13.00 & & & 44.6 & 439 & 0.028 & 0.010 & 10 & 85 & -0.61 & 0.35 & 14.4 & 34.4 \\
\hline $090700+0654$ & 13.35 & 1.57 & M4 e & 20.8 & 373 & 0.127 & 0.020 & 73 & 28 & -0.51 & 0.13 & 71.4 & 37.1 \\
\hline HD 78727 & 8.38 & 1.01 & $\mathrm{~K} 4$ & 21.5 & 369 & 0.045 & 0.015 & 15 & 17 & -0.06 & 0.31 & 36.0 & 19.9 \\
\hline HD 78899 & 7.68 & & G5 & 36.8 & 484 & 0.239 & 0.024 & 224 & 25 & -0.25 & 0.09 & 166.9 & 270.0 \\
\hline Gl $337 \mathrm{~A}$ & 7.25 & 0.73 & $\mathrm{~K} 0 \mathrm{~V}$ & 20.5 & 189 & 0.061 & 0.022 & 14 & 19 & -1.00 & 0.45 & 18.5 & 9.3 \\
\hline Gl 337 B & 7.30 & & & 20.5 & 189 & 0.061 & 0.022 & 14 & 19 & -1.00 & 0.45 & 18.5 & 9.3 \\
\hline Gl $338 \mathrm{~A}$ & 7.62 & 1.39 & M0 Ve & 6.2 & 326 & 0.257 & 0.030 & 149 & 3 & -0.67 & 0.09 & 122.3 & 5.6 \\
\hline Gl 338 B & 7.71 & 1.42 & M0 Ve & 6.1 & 326 & 0.257 & 0.030 & 149 & 17 & -0.67 & 0.09 & 122.3 & 5.5 \\
\hline Gl $338.1 \mathrm{~A}$ & 10.67 & 1.38 & K5 & 27.2 & 470 & 0.020 & 0.008 & 9 & 33 & -0.27 & 0.36 & 13.4 & 11.9 \\
\hline Gl $338.1 B$ & 11.00 & & & 27.2 & 470 & 0.020 & 0.008 & 9 & 33 & -0.27 & 0.36 & 13.4 & 11.9 \\
\hline Gl 339 AB & 8.04 & 1.02 & dK5 & 18.4 & 368 & 0.423 & 0.037 & 373 & 5 & -0.36 & 0.08 & 270.9 & 109.9 \\
\hline Gl 339.3 & 4.62 & 0.45 & F5 III & 50.8 & 572 & 0.038 & 0.011 & 9 & 51 & -0.56 & 0.30 & 20.1 & 62.1 \\
\hline LTT 3412 & 10.75 & 1.55 & M2 & 12.7 & 352 & 0.044 & 0.013 & 27 & 31 & -0.35 & 0.25 & 28.4 & 5.5 \\
\hline HD 79873 & 6.71 & 0.41 & F6 III-IV & 69.0 & 338 & 0.126 & 0.021 & 56 & 15 & -0.14 & 0.16 & 95.2 & 541.8 \\
\hline Gl $340 \mathrm{~A}$ & 7.86 & 1.00 & K3 V & 17.5 & 340 & 0.041 & 0.014 & 11 & 36 & -0.74 & 0.27 & 18.2 & 6.7 \\
\hline Gl 340 B & 8.10 & & K3 V & 17.5 & 340 & 0.041 & 0.014 & 11 & 36 & -0.74 & 0.27 & 18.2 & 6.7 \\
\hline $\mathrm{BD}+271739$ & 9.55 & 1.34 & K5 & 19.3 & 364 & 0.027 & 0.011 & 10 & 15 & -0.19 & 0.35 & 19.7 & 8.8 \\
\hline G235-020 & 11.28 & & $\mathrm{~m}$ & 32.5 & 350 & 0.200 & 0.026 & 149 & 10 & -0.16 & 0.13 & 149.2 & 188.1 \\
\hline GJ $1122 \mathrm{~A}$ & 14.52 & 1.68 & $\mathrm{~m}$ & 20.2 & 459 & 0.034 & 0.012 & 14 & 8 & -0.61 & 0.20 & 17.5 & 8.6 \\
\hline GJ $1122 \mathrm{~B}$ & 14.67 & 1.68 & $\mathrm{~m}$ & 20.2 & 459 & 0.034 & 0.012 & 14 & 14 & -0.61 & 0.20 & 17.5 & 8.6 \\
\hline Gl $340.1 \mathrm{~A}$ & 6.13 & 0.42 & $\mathrm{~F} 4 \mathrm{~V}$ & 33.6 & 297 & 0.049 & 0.015 & 19 & 9 & -0.32 & 0.27 & 32.2 & 43.4 \\
\hline Gl 340.1B & 6.13 & & & 33.6 & 297 & 0.049 & 0.015 & 19 & 9 & -0.32 & 0.27 & 32.2 & 43.4 \\
\hline G115-071 & 14.02 & & $\mathrm{~m}$ & 15.4 & 468 & 0.032 & 0.011 & 19 & 7 & 0.08 & 0.33 & 28.3 & 8.0 \\
\hline GJ 1124 & 7.63 & 0.99 & $\mathrm{~K} 2 \mathrm{~V}$ & 24.3 & 450 & 2.908 & 0.082 & 999 & 8 & -0.02 & 0.03 & 2385.7 & 1681.6 \\
\hline Gl 342 & 9.03 & 1.19 & dK5 e & 20.1 & 497 & 0.040 & 0.011 & 17 & 10 & -1.00 & 0.15 & 12.0 & 5.8 \\
\hline Gl 343.1 & 9.84 & 1.29 & dK8 e & 32.3 & 430 & 0.100 & 0.017 & 52 & 14 & -0.30 & 0.16 & 67.2 & 83.7 \\
\hline Gl 344 A & 5.80 & 0.65 & G2 V & 31.2 & 395 & 0.030 & 0.012 & 9 & 22 & -0.89 & 0.27 & 10.7 & 12.6 \\
\hline Gl 344 B & 6.60 & & & 31.2 & 395 & 0.030 & 0.012 & 9 & 22 & -0.89 & 0.27 & 10.7 & 12.6 \\
\hline Gl 346 & 10.52 & 1.43 & dM0 & 20.5 & 364 & 0.044 & 0.014 & 17 & 13 & -0.03 & 0.30 & 35.9 & 18.1 \\
\hline Gl 348 A & 4.60 & 0.45 & F6 V & 17.1 & 365 & 0.574 & 0.041 & 507 & 18 & -0.26 & 0.07 & 398.0 & 139.1 \\
\hline Gl 348 B & 7.18 & 0.87 & K0 & 17.1 & 365 & 0.574 & 0.041 & 507 & 48 & -0.26 & 0.07 & 398.0 & 139.1 \\
\hline Gl 349 & 7.20 & 1.00 & $\mathrm{~K} 3 \mathrm{Ve}$ & 12.7 & 385 & 0.184 & 0.023 & 136 & 4 & -0.51 & 0.11 & 103.3 & 19.9 \\
\hline HD 81937 & 3.67 & 0.33 & F0 IV & 23.1 & 346 & 0.042 & 0.013 & 14 & 9 & -1.00 & 0.45 & 12.7 & 8.2 \\
\hline Gl 351 A & 4.12 & 0.36 & F3 IV & 18.6 & 427 & 0.558 & 0.038 & 552 & 6 & -0.34 & 0.06 & 363.3 & 149.6 \\
\hline Gl 351 B & 4.65 & & F0 IV & 18.6 & 427 & 0.558 & 0.038 & 552 & 6 & -0.34 & 0.06 & 363.3 & 149.6 \\
\hline Gl $352 \mathrm{~A}$ & 10.81 & 1.53 & M3 & 10.5 & 402 & 0.018 & 0.009 & 8 & 10 & -0.43 & 0.32 & 10.7 & 1.4 \\
\hline Gl 352 B & 10.80 & & & 10.5 & 402 & 0.018 & 0.009 & 8 & 10 & -0.43 & 0.32 & 10.7 & 1.4 \\
\hline Gl $354.1 \mathrm{~A}$ & 7.01 & 0.77 & dG9 & 17.8 & 434 & 0.731 & 0.043 & 770 & 5 & -0.20 & 0.06 & 530.3 & 200.2 \\
\hline Gl 354.1B & 16.50 & & $\mathrm{~m}$ & 17.8 & 434 & 0.731 & 0.043 & 770 & 57 & -0.20 & 0.06 & 530.3 & 200.2 \\
\hline Gl 355 & 7.82 & 0.92 & K0 & 18.3 & 445 & 2.730 & 0.202 & 583 & 10 & -0.04 & 0.07 & 2210.8 & 890.5 \\
\hline Gl 355.1 & 4.56 & 0.77 & G4 III-IV & 32.4 & 419 & 2.029 & 0.071 & 999 & 3 & 0.05 & 0.03 & 1739.9 & 2180.2 \\
\hline Gl 356 A & 5.41 & 0.77 & G8 V & 11.2 & 467 & 0.298 & 0.027 & 254 & 27 & -0.55 & 0.08 & 160.9 & 24.1 \\
\hline Gl 356 B & 13.00 & & & 11.2 & 467 & 0.298 & 0.027 & 254 & 27 & -0.55 & 0.08 & 160.9 & 24.1 \\
\hline Wo 9303 & 11.02 & 1.44 & K5 & 33.2 & 431 & 0.075 & 0.017 & 22 & 27 & -0.18 & 0.20 & 55.1 & 72.8 \\
\hline Gl 358 & 10.75 & 1.53 & M3 & 9.5 & 410 & 0.175 & 0.023 & 118 & 17 & -0.31 & 0.13 & 116.8 & 12.6 \\
\hline Gl 360 & 10.57 & 1.50 & M3 & 11.8 & 299 & 0.179 & 0.029 & 70 & 68 & -0.40 & 0.13 & 110.7 & 18.3 \\
\hline Gl 361 & 10.37 & 1.50 & $\mathrm{dM} 2 \mathrm{e}$ & 11.4 & 409 & 0.038 & 0.012 & 16 & 9 & -0.42 & 0.28 & 23.2 & 3.6 \\
\hline Gl 362 & 11.22 & 1.52 & M3 & 11.5 & 299 & 0.179 & 0.029 & 70 & 33 & -0.40 & 0.13 & 110.7 & 17.6 \\
\hline Gl 365 & 8.12 & 1.15 & K5 V & 17.8 & 413 & 0.035 & 0.013 & 10 & 18 & -0.67 & 0.24 & 16.5 & 6.2 \\
\hline G042-024 & 14.05 & & $\mathrm{~m}$ & 10.3 & 361 & 0.023 & 0.010 & 8 & 11 & -0.51 & 0.32 & 13.2 & 1.7 \\
\hline Gl 373 & 9.00 & 1.43 & dM1 & 10.5 & 596 & 0.059 & 0.012 & 44 & 8 & -0.52 & 0.17 & 32.7 & 4.3 \\
\hline G116-065 & 12.73 & & M4 & 19.0 & 445 & 0.105 & 0.019 & 57 & 25 & -0.41 & 0.14 & 64.2 & 27.7 \\
\hline HD 86146 & 5.14 & 0.46 & F6 Vas & 28.9 & 366 & 0.087 & 0.019 & 31 & 9 & -0.67 & 0.15 & 41.5 & 41.5 \\
\hline G116-072 & 13.89 & & $\mathrm{~m}$ & 22.6 & 484 & 0.216 & 0.024 & 149 & 5 & -0.26 & 0.10 & 150.0 & 91.9 \\
\hline G116-073 & 14.16 & & $\mathrm{~m}$ & 22.6 & 484 & 0.216 & 0.024 & 149 & 21 & -0.26 & 0.10 & 150.0 & 91.9 \\
\hline Gl 375 & 11.27 & 1.55 & M3.5 & 15.9 & 394 & 0.805 & 0.067 & 431 & 3 & -0.17 & 0.08 & 596.5 & 180.4 \\
\hline HD 86590 & 7.90 & 0.88 & $\mathrm{~K} 0 \mathrm{~V}$ & 32.5 & 452 & 2.053 & 0.068 & 999 & 12 & -0.02 & 0.03 & 1684.3 & 2124.2 \\
\hline Gl 378.1 & 9.04 & 1.07 & $\mathrm{dK} 8$ & 27.7 & 507 & 0.055 & 0.014 & 21 & 14 & -0.63 & 0.16 & 27.5 & 25.2 \\
\hline Gl 378.2 & 9.95 & 1.39 & dM0 e & 22.3 & 435 & 0.023 & 0.009 & 9 & 22 & -0.32 & 0.35 & 15.3 & 9.1 \\
\hline Gl 380 & 6.59 & 1.36 & $\mathrm{~K} 2 \mathrm{Ve}$ & 4.9 & 494 & 0.177 & 0.021 & 153 & 9 & -0.54 & 0.09 & 96.3 & 2.7 \\
\hline Gl 382 & 9.27 & 1.48 & $\mathrm{dM} 2$ & 7.8 & 430 & 0.065 & 0.015 & 32 & 2 & -0.67 & 0.19 & 30.9 & 2.3 \\
\hline Gl 384 A & 8.27 & 0.80 & G8 V & 41.0 & 354 & 0.047 & 0.014 & 18 & 10 & -0.04 & 0.28 & 38.3 & 77.0 \\
\hline Gl 384 B & 10.90 & & & 41.0 & 354 & 0.047 & 0.014 & 18 & 10 & -0.04 & 0.28 & 38.3 & 77.0 \\
\hline Gl 385.1 & 6.38 & 0.59 & G1 V & 22.7 & 281 & 0.080 & 0.021 & 23 & 35 & -0.14 & 0.24 & 60.8 & 37.6 \\
\hline Wo 9321 & 7.21 & 0.49 & $\mathrm{dF} 6$ & 48.3 & 379 & 0.134 & 0.020 & 94 & 4 & 0.00 & 0.16 & 111.4 & 311.2 \\
\hline GJ 2079 & 10.20 & 1.36 & dM0 e & 20.4 & 300 & 0.507 & 0.043 & 353 & 11 & -0.38 & 0.08 & 319.5 & 159.2 \\
\hline Gl 388 & 9.40 & 1.54 & $\mathrm{M} 4.5 \mathrm{Ve}$ & 4.9 & 104 & 3.701 & 0.194 & 999 & 7 & -0.28 & 0.05 & 2526.3 & 72.2 \\
\hline Gl 388.2 & 7.18 & 0.55 & F8 V & 34.6 & 398 & 0.035 & 0.011 & 17 & 4 & -0.82 & 0.18 & 13.9 & 19.9 \\
\hline Gl 390 & 10.17 & 1.49 & M1.5 & 12.5 & 401 & 0.016 & 0.007 & 9 & 9 & -0.71 & 0.54 & 7.3 & 1.4 \\
\hline *Gl 391 & 4.00 & 0.35 & F2 IV & 16.2 & 606 & 0.100 & 0.013 & 148 & 42 & $-0.44:$ & $0.21:$ & 59.9: & 18.8: \\
\hline Gl 392.1 & 5.26 & 0.37 & $\mathrm{~F} 2 \mathrm{~V}$ & 21.5 & 329 & 0.309 & 0.033 & 181 & 6 & -0.21 & 0.11 & 222.5 & 123.1 \\
\hline G118-061 & 13.00 & & $\mathrm{~m}$ & 19.1 & 463 & 0.019 & 0.009 & 7 & 8 & -0.50 & 0.31 & 11.0 & 4.8 \\
\hline Gl 393 & 9.64 & 1.52 & $\mathrm{dM} 2.5$ & 7.2 & 401 & 0.025 & 0.011 & 10 & 10 & -0.73 & 0.21 & 11.2 & 0.7 \\
\hline Gl 395 & 4.84 & 0.52 & F8 V & 12.9 & 572 & 0.195 & 0.021 & 172 & 5 & -0.68 & 0.07 & 92.0 & 18.2 \\
\hline Gl 396 & 7.30 & 0.82 & K0 & 21.1 & 589 & 0.032 & 0.009 & 18 & 12 & -0.64 & 0.20 & 15.9 & 8.4 \\
\hline Gl 397 & 8.86 & 1.33 & $\mathrm{~K} 7 \mathrm{~V}$ & 15.7 & 392 & 0.060 & 0.015 & 20 & 18 & -0.36 & 0.23 & 38.3 & 11.3 \\
\hline
\end{tabular}




\begin{tabular}{|c|c|c|c|c|c|c|c|c|c|c|c|c|c|}
\hline Name & $V$ & $B-V$ & Sp. type & $d(\mathrm{pc})$ & Exp. & $C R$ & $\pm C R$ & $\mathrm{Li}$ & $\Delta$ & $h r$ & $\Delta h r$ & $f_{\mathrm{x}-14}$ & $L_{\mathrm{x} 27}$ \\
\hline Gl $397.1 \mathrm{~A}$ & 9.65 & 1.38 & dM0 e & 17.5 & 543 & 0.038 & 0.010 & 25 & 6 & -0.75 & 0.16 & 16.6 & 6.1 \\
\hline BD-20 3198 & 10.68 & 1.38 & K7 V & 29.2 & 256 & 0.149 & 0.027 & 58 & 16 & -0.19 & 0.18 & 109.1 & 111.0 \\
\hline DON $414 \mathrm{C}$ & 13.13 & 1.57 & & 29.2 & 256 & 0.149 & 0.027 & 58 & 16 & -0.19 & 0.18 & 109.1 & 111.0 \\
\hline Gl 398 & 12.60 & 1.60 & $\mathrm{dM} 4 \mathrm{e}$ & 14.5 & 165 & 0.205 & 0.039 & 48 & 39 & -0.26 & 0.19 & 142.2 & 36.0 \\
\hline LP $167-71$ & 13.49 & & $\mathrm{~m}$ & 22.4 & 542 & 0.068 & 0.014 & 39 & 5 & -0.58 & 0.15 & 35.8 & 21.4 \\
\hline GJ $1136 \mathrm{~A}$ & 10.19 & 1.46 & $\mathrm{~K} 7 \mathrm{~V}$ & 16.3 & 297 & 0.048 & 0.016 & 11 & 24 & -0.87 & 0.16 & 17.9 & 5.7 \\
\hline GJ 1136 B & 11.67 & 1.52 & & 16.3 & 297 & 0.048 & 0.016 & 11 & 24 & -0.87 & 0.16 & 17.9 & 5.7 \\
\hline HD 92945 & 7.74 & 0.89 & K1 V & 21.6 & 361 & 0.118 & 0.020 & 68 & 9 & -0.13 & 0.17 & 89.6 & 49.8 \\
\hline Gl $400 \mathrm{~A}$ & 9.30 & 1.41 & $\mathrm{dM} 2$ & 14.1 & 455 & 0.022 & 0.009 & 8 & 4 & -1.00 & 0.35 & 6.5 & 1.6 \\
\hline $\mathrm{Gl} 400 \mathrm{~B}$ & 12.20 & & & 14.1 & 455 & 0.022 & 0.009 & 8 & 4 & -1.00 & 0.35 & 6.5 & 1.6 \\
\hline HD 93811 & 8.33 & & G5 & 28.7 & 548 & 0.157 & 0.020 & 110 & 11 & -0.22 & 0.11 & 112.0 & 110.7 \\
\hline LP $263-35$ & 13.46 & & $\mathrm{~m}$ & 24.5 & 327 & 0.127 & 0.022 & 73 & 6 & -0.03 & 0.17 & 103.4 & 74.3 \\
\hline G045-011 & 13.85 & & $\mathrm{~m}$ & 15.9 & 415 & 0.186 & 0.024 & 138 & 9 & -0.09 & 0.13 & 146.1 & 44.0 \\
\hline G044-043 & 14.40 & & $\mathrm{~m}$ & 19.0 & 333 & 0.071 & 0.017 & 33 & 17 & -0.26 & 0.21 & 48.9 & 21.1 \\
\hline Wo 9336 & 7.05 & 0.64 & $\mathrm{G} 3 / 5 \mathrm{~V}$ & 41.3 & 345 & 0.145 & 0.024 & 94 & 0 & 0.02 & 0.16 & 121.6 & 248.4 \\
\hline Gl 403.1 & 5.23 & 0.46 & $\mathrm{~F} 6 \mathrm{~V}$ & 31.3 & 289 & 0.070 & 0.018 & 37 & 15 & 0.14 & 0.24 & 63.7 & 74.9 \\
\hline Gl 404.1 & 3.78 & 0.95 & K1 III & 29.7 & 192 & 0.135 & 0.029 & 45 & 4 & -0.05 & 0.21 & 108.5 & 114.3 \\
\hline HD 94765 & 7.34 & 0.92 & K0 & 17.5 & 344 & 0.153 & 0.022 & 87 & 5 & -0.36 & 0.15 & 98.2 & 36.2 \\
\hline Gl 406 & 13.45 & 2.00 & M6 & 2.4 & 384 & 0.227 & 0.026 & 183 & 18 & -0.22 & 0.11 & 162.5 & 1.1 \\
\hline G147-011 & 15.36 & 1.75 & $\mathrm{~m}$ & 22.3 & 352 & 0.020 & 0.010 & 8 & 13 & -0.52 & 0.35 & 11.4 & 6.8 \\
\hline Gl 408 & 10.02 & 1.54 & M3 & 6.6 & 212 & 0.026 & 0.013 & 8 & 39 & -1.00 & 1.51 & 8.0 & 0.4 \\
\hline Gl 410 & 9.60 & 1.48 & $\mathrm{dM} 2 \mathrm{e}$ & 11.7 & 178 & 0.240 & 0.039 & 87 & 9 & -0.42 & 0.16 & 146.3 & 23.8 \\
\hline LP $263-64$ & 13.67 & & $\mathrm{~m}$ & 21.1 & 360 & 0.108 & 0.020 & 53 & 7 & -0.30 & 0.17 & 72.8 & 38.8 \\
\hline Gl 411 & 7.48 & 1.51 & $\mathrm{M} 2 \mathrm{Ve}$ & 2.5 & 341 & 0.167 & 0.024 & 83 & 10 & -0.67 & 0.10 & 79.5 & 0.6 \\
\hline HD 96202 & 5.63 & 0.37 & F3 IV & 43.5 & 289 & 0.032 & 0.013 & 12 & 8 & -1.00 & 0.26 & 9.6 & 21.6 \\
\hline Gl $412 \mathrm{~A}$ & 8.74 & 1.54 & M2 Ve & 4.8 & 364 & 0.185 & 0.027 & 111 & 25 & -0.65 & 0.09 & 89.9 & 2.5 \\
\hline $\mathrm{Gl} 412 \mathrm{~B}$ & 14.40 & 2.09 & M6 e & 4.8 & 364 & 0.185 & 0.027 & 111 & 13 & -0.65 & 0.09 & 89.9 & 2.5 \\
\hline Gl $414 \mathrm{~A}$ & 8.33 & 1.34 & K8 V & 11.9 & 317 & 0.046 & 0.016 & 10 & 36 & -0.80 & 0.18 & 18.7 & 3.2 \\
\hline $\mathrm{Gl} 414 \mathrm{~B}$ & 9.98 & 1.52 & $\mathrm{M} 2 \mathrm{~V}$ & 11.9 & 317 & 0.046 & 0.016 & 10 & 36 & -0.80 & 0.18 & 18.7 & 3.2 \\
\hline G119-062 & 12.38 & & dM4 & 14.6 & 262 & 0.366 & 0.040 & 201 & 4 & -0.21 & 0.11 & 263.3 & 67.5 \\
\hline Gl 416.1 & 4.48 & 0.03 & A2 III & 81.3 & 293 & 0.469 & 0.043 & 206 & 11 & -1.00 & 0.04 & 141.1 & 1116.2 \\
\hline Gl 417 & 6.41 & 0.61 & G0 V & 21.7 & 207 & 0.320 & 0.045 & 114 & 11 & -0.26 & 0.13 & 222.0 & 125.5 \\
\hline Gl 418 & 8.69 & 1.18 & K5 V & 18.0 & 422 & 0.036 & 0.011 & 17 & 8 & -0.91 & 0.22 & 12.6 & 4.9 \\
\hline Gl $420 \mathrm{~A}$ & 7.68 & 1.06 & dK5 & 14.7 & 483 & 0.117 & 0.018 & 66 & 14 & -0.40 & 0.13 & 72.5 & 18.7 \\
\hline $\mathrm{Gl} 420 \mathrm{~B}$ & 11.40 & & M2 & 14.7 & 483 & 0.117 & 0.018 & 66 & 14 & -0.40 & 0.13 & 72.5 & 18.7 \\
\hline Steph 932 & 11.22 & 1.41 & M0 & 23.8 & 547 & 0.323 & 0.027 & 337 & 7 & -0.25 & 0.08 & 225.7 & 152.3 \\
\hline Gl $421.1 \mathrm{~A}$ & 6.50 & 0.43 & $\mathrm{~F} 6 \mathrm{~V}$ & 39.2 & 384 & 0.034 & 0.012 & 11 & 85 & -0.69 & 0.32 & 15.7 & 28.9 \\
\hline Gl 421.1B & 8.03 & 0.60 & F9 V & 45.2 & 384 & 0.034 & 0.012 & 11 & 87 & -0.69 & 0.32 & 15.7 & 38.4 \\
\hline $\mathrm{Gl} 423 \mathrm{~A}$ & 4.33 & 0.59 & G0 Ve & 8.8 & 192 & 4.539 & 0.157 & 999 & 7 & -0.21 & 0.03 & 3266.7 & 305.0 \\
\hline Gl $423 \mathrm{~B}$ & 4.80 & & G0 Ve & 8.8 & 192 & 4.539 & 0.157 & 999 & 7 & -0.21 & 0.03 & 3266.7 & 305.0 \\
\hline Gl 424 & 9.31 & 1.41 & M1 V & 9.1 & 619 & 0.018 & 0.007 & 9 & 17 & -0.63 & 0.30 & 9.0 & 0.9 \\
\hline Gl $425 \mathrm{~A}$ & 8.74 & 1.36 & $\mathrm{~K} 4 / 5 \mathrm{~V}$ & 13.2 & 292 & 0.914 & 0.057 & 716 & 11 & -0.13 & 0.06 & 696.3 & 144.2 \\
\hline $\mathrm{Gl} 425 \mathrm{~B}$ & 11.00 & & $\mathrm{~m}$ & 13.2 & 292 & 0.914 & 0.057 & 716 & 11 & -0.13 & 0.06 & 696.3 & 144.2 \\
\hline GJ $2084 \mathrm{AB}$ & 9.08 & 1.26 & $\mathrm{~K} 4 \mathrm{~V}$ & 46.7 & 330 & 0.656 & 0.046 & 529 & 9 & 0.06 & 0.07 & 566.3 & 1479.6 \\
\hline G253-050 & 12.15 & & M2.5 & 18.4 & 520 & 0.021 & 0.008 & 8 & 67 & -1.00 & 0.25 & 6.3 & 2.5 \\
\hline G253-049 & 12.65 & & M3 & 27.2 & 520 & 0.021 & 0.008 & 8 & 27 & -1.00 & 0.25 & 6.3 & 5.6 \\
\hline $\mathrm{Gl} 428 \mathrm{~A}$ & 7.50 & 1.26 & $\mathrm{~K} 7 \mathrm{~V}$ & 12.9 & 209 & 0.076 & 0.022 & 25 & 13 & -0.43 & 0.27 & 45.7 & 9.1 \\
\hline $\mathrm{Gl} 428 \mathrm{~B}$ & 8.30 & & Mo Ve & 12.9 & 209 & 0.076 & 0.022 & 25 & 13 & -0.43 & 0.27 & 45.7 & 9.1 \\
\hline Gl $429 \mathrm{~A}$ & 6.50 & 0.80 & K0 IV & 17.7 & 386 & 0.030 & 0.012 & 7 & 39 & -0.99 & 0.21 & 9.2 & 3.4 \\
\hline Gl $429 \mathrm{~B}$ & 7.58 & 1.00 & K2 IV-V & 18.0 & 386 & 0.030 & 0.012 & 7 & 64 & -0.99 & 0.21 & 9.2 & 3.6 \\
\hline Gl 429.4 & 8.33 & 1.06 & $\mathrm{~K} 4 \mathrm{Ve}$ & 22.2 & 137 & 0.076 & 0.027 & 11 & 19 & -0.52 & 0.35 & 42.3 & 25.0 \\
\hline HD 100180 & 6.20 & 0.57 & G0 V & 23.0 & 398 & 0.031 & 0.010 & 16 & 13 & -1.00 & 1.22 & 9.2 & 5.9 \\
\hline LTT 13146 & 9.22 & 1.14 & K6 IV & 23.0 & 398 & 0.031 & 0.010 & 16 & 13 & -1.00 & 1.22 & 9.2 & 5.9 \\
\hline Gl 431 & 11.52 & 1.54 & M3.5 & 10.5 & 210 & 0.662 & 0.060 & 440 & 8 & -0.38 & 0.08 & 417.0 & 54.7 \\
\hline Wo 9367 & 5.77 & 0.46 & F6 V & 26.5 & 417 & 0.242 & 0.027 & 197 & 7 & -0.33 & 0.10 & 158.7 & 133.6 \\
\hline G122-036 & 10.03 & 1.35 & K5 & 24.4 & 248 & 0.028 & 0.013 & 9 & 6 & -0.98 & 0.79 & 8.8 & 6.3 \\
\hline HD 101206 & 8.26 & 0.96 & K5 V & 19.8 & 255 & 0.107 & 0.024 & 30 & 22 & 0.32 & 0.22 & 107.1 & 50.0 \\
\hline Gl 434 & 5.33 & 0.72 & G8 Ve & 9.5 & 314 & 0.348 & 0.037 & 194 & 15 & -0.76 & 0.07 & 149.0 & 16.2 \\
\hline Gl 436 & 10.67 & 1.52 & dM3.5 & 10.2 & 455 & 0.018 & 0.008 & 7 & 58 & -1.00 & 2.26 & 5.4 & 0.7 \\
\hline Gl 441 & 9.02 & 1.17 & $\mathrm{dK} 8$ & 27.5 & 759 & 0.022 & 0.007 & 17 & 10 & -0.49 & 0.25 & 12.7 & 11.6 \\
\hline G236-081 & 13.60 & & $\mathrm{~m}$ & 16.5 & 805 & 0.094 & 0.013 & 88 & 12 & -0.23 & 0.13 & 66.4 & 21.7 \\
\hline G010-049 & 13.25 & & $\mathrm{~m}$ & 14.6 & 373 & 0.114 & 0.020 & 75 & 11 & -0.24 & 0.16 & 80.1 & 20.5 \\
\hline LP $613-50$ & 17.60 & & $\mathrm{~m}$ & 14.6 & 373 & 0.114 & 0.020 & 75 & 22 & -0.24 & 0.16 & 80.1 & 20.5 \\
\hline Gl 447 & 11.12 & 1.75 & $\mathrm{dM} 4.5$ & 3.3 & 364 & 0.080 & 0.017 & 41 & 3 & -0.34 & 0.19 & 51.8 & 0.7 \\
\hline Gl 449 & 3.61 & 0.55 & F9 V & 10.9 & 417 & 0.344 & 0.030 & 264 & 11 & -0.63 & 0.07 & 170.9 & 24.3 \\
\hline Gl 450 & 9.73 & 1.51 & M1 Ve & 8.6 & 377 & 0.069 & 0.015 & 37 & 6 & -0.19 & 0.20 & 50.2 & 4.4 \\
\hline Gl 452.4 & 10.52 & 1.39 & Mo Ve & 28.7 & 395 & 0.019 & 0.009 & 9 & 12 & -0.75 & 0.33 & 8.2 & 8.1 \\
\hline Gl $452.5 \mathrm{~A}$ & 7.29 & 0.64 & G5 V & 31.0 & 282 & 0.234 & 0.032 & 128 & 4 & -0.28 & 0.13 & 159.9 & 183.3 \\
\hline Gl $452.5 B$ & 7.70 & & & 31.0 & 282 & 0.234 & 0.032 & 128 & 4 & -0.28 & 0.13 & 159.9 & 183.3 \\
\hline Gl $454.2 \mathrm{~A}$ & 7.35 & 0.59 & G0 V & 56.2 & 163 & 0.162 & 0.034 & 61 & 12 & 0.32 & 0.20 & 162.5 & 613.6 \\
\hline Gl $454.2 B$ & 8.10 & & & 56.2 & 163 & 0.162 & 0.034 & 61 & 12 & 0.32 & 0.20 & 162.5 & 613.6 \\
\hline Gl 455 & 12.84 & 1.75 & M3 & 20.2 & 478 & 0.089 & 0.017 & 38 & 10 & -0.75 & 0.13 & 38.6 & 18.9 \\
\hline HD 104731 & 5.15 & 0.41 & F6 V & 24.2 & 173 & 0.067 & 0.023 & 14 & 30 & -0.32 & 0.31 & 44.4 & 31.1 \\
\hline Gl 455.1 & 9.99 & 1.34 & K5 V & 22.5 & 92 & 0.125 & 0.042 & 13 & 29 & 0.20 & 0.32 & 117.4 & 71.3 \\
\hline$* \mathrm{Gl} 455.2$ & 4.15 & 0.34 & F2 III & 19.7 & 374 & 0.372 & 0.032 & 522 & 2 & -0.05 & 0.09 & 299.3 & 139.0 \\
\hline Gl 455.3 & 4.02 & 0.32 & F2 III-IV & 14.8 & 292 & 1.362 & 0.100 & 663 & 8 & -0.34 & 0.07 & 886.4 & 231.4 \\
\hline Wo $9390 \mathrm{~A}$ & 6.81 & 0.66 & G2 V & 26.5 & 408 & 0.025 & 0.010 & 8 & 52 & -1.00 & 1.35 & 7.5 & 6.3 \\
\hline Wo $9390 \mathrm{~B}$ & 9.20 & & K5 & 26.5 & 408 & 0.025 & 0.010 & 8 & 33 & -1.00 & 1.35 & 7.5 & 6.3 \\
\hline HD 105631 & 7.47 & 0.79 & $\mathrm{~K} 0 \mathrm{~V}$ & 24.3 & 445 & 0.039 & 0.012 & 14 & 6 & -0.70 & 0.21 & 18.0 & 12.8 \\
\hline Gl $456.1 \mathrm{~A}$ & 8.45 & 1.14 & K5 V & 20.1 & 279 & 0.043 & 0.015 & 13 & 20 & -0.72 & 0.26 & 19.2 & 9.3 \\
\hline
\end{tabular}




\begin{tabular}{|c|c|c|c|c|c|c|c|c|c|c|c|c|c|}
\hline Name & $V$ & $B-V$ & Sp. type & $d(\mathrm{pc})$ & Exp. & $C R$ & $\pm C R$ & $\mathrm{Li}$ & $\Delta$ & $h r$ & $\Delta h r$ & $f_{\mathrm{x}-14}$ & $L_{\mathrm{x} 27}$ \\
\hline Gl 456.1B & 13.25 & 1.56 & $\mathrm{~m}$ & 20.1 & 279 & 0.043 & 0.015 & 13 & 43 & -0.72 & 0.26 & 19.2 & 9.3 \\
\hline HD 105963 & 8.03 & 0.88 & $\mathrm{~K} 2$ & 26.5 & 421 & 0.150 & 0.021 & 96 & 11 & -0.41 & 0.12 & 92.2 & 77.6 \\
\hline LTT 13390 & 8.23 & & K3 & 31.5 & 421 & 0.150 & 0.021 & 96 & 23 & -0.41 & 0.12 & 92.2 & 109.7 \\
\hline G122-074 & 12.91 & & $\mathrm{~m}$ & 21.8 & 318 & 0.072 & 0.018 & 28 & 10 & -0.15 & 0.24 & 53.9 & 30.6 \\
\hline GJ $1154 \mathrm{AB}$ & 13.73 & 1.77 & $\mathrm{~m}$ & 8.4 & 332 & 0.098 & 0.020 & 43 & 12 & -0.24 & 0.19 & 69.2 & 5.8 \\
\hline San 39 & 14.15 & 1.62 & $\mathrm{dM} 4-5 \mathrm{e}$ & 19.7 & 397 & 0.051 & 0.014 & 25 & 20 & -0.52 & 0.19 & 28.1 & 13.1 \\
\hline GJ 1156 & 13.81 & 1.88 & $\mathrm{dM} \mathrm{e}$ & 6.5 & 400 & 0.131 & 0.020 & 67 & 6 & -0.25 & 0.15 & 91.3 & 4.7 \\
\hline HD 108421 & 8.89 & 1.02 & K3 V & 27.5 & 439 & 0.032 & 0.012 & 11 & 14 & -0.50 & 0.24 & 18.3 & 16.7 \\
\hline G148-059 & 14.88 & 1.73 & dM5 e & 27.5 & 448 & 0.027 & 0.010 & 9 & 9 & -0.33 & 0.31 & 18.0 & 16.3 \\
\hline LTT 4730 & 10.96 & 1.51 & M2 & 17.3 & 58 & 0.218 & 0.066 & 17 & 71 & -0.32 & 0.29 & 143.9 & 51.7 \\
\hline LP 735-11 & 11.00 & & $\mathrm{~g}$ & 17.3 & 58 & 0.218 & 0.066 & 17 & 56 & -0.32 & 0.29 & 143.9 & 51.7 \\
\hline G123-035 & 12.90 & & $\mathrm{~m}$ & 14.4 & 487 & 0.146 & 0.020 & 94 & 8 & -0.39 & 0.12 & 91.1 & 22.6 \\
\hline LP $377-100$ & 14.18 & 1.60 & dM5 & 20.4 & 459 & 0.092 & 0.016 & 57 & 9 & 0.21 & 0.18 & 87.0 & 43.3 \\
\hline Gl $469.2 \mathrm{~A}$ & 6.46 & 0.58 & G0 V & 25.0 & 257 & 0.620 & 0.050 & 432 & 4 & -0.19 & 0.08 & 452.9 & 338.6 \\
\hline Gl $469.2 \mathrm{~B}$ & 9.20 & & & 25.0 & 257 & 0.620 & 0.050 & 432 & 4 & -0.19 & 0.08 & 452.9 & 338.6 \\
\hline GJ 1160 & 8.12 & 0.93 & $\mathrm{~K} 2 \mathrm{~V}$ & 23.8 & 392 & 0.060 & 0.015 & 21 & 17 & -0.04 & 0.24 & 48.5 & 32.7 \\
\hline Gl 471.2 & 4.30 & 0.37 & F0 IV & 18.2 & 231 & 0.209 & 0.032 & 86 & 4 & -0.48 & 0.13 & 120.5 & 47.8 \\
\hline Steph 1021 & 12.90 & 1.56 & M4 & 21.1 & 439 & 0.127 & 0.020 & 88 & 6 & -0.27 & 0.14 & 87.7 & 46.7 \\
\hline Gl $473 \mathrm{~A}$ & 13.04 & 1.83 & $\mathrm{dM} 5.5 \mathrm{e}$ & 4.4 & 430 & 0.239 & 0.027 & 147 & 12 & -0.44 & 0.09 & 142.6 & 3.3 \\
\hline Gl 473 B & 13.30 & & M7 & 4.4 & 430 & 0.239 & 0.027 & 147 & 12 & -0.44 & 0.09 & 142.6 & 3.3 \\
\hline Gl 475 & 4.27 & 0.59 & G0 V & 8.4 & 509 & 0.023 & 0.008 & 12 & 40 & -1.00 & 0.39 & 7.0 & 0.6 \\
\hline GJ $1161 \mathrm{~A}$ & 7.91 & 1.04 & $\mathrm{~K} 4 \mathrm{~V}$ & 21.6 & 220 & 0.050 & 0.021 & 7 & 20 & -0.86 & 0.30 & 18.9 & 10.6 \\
\hline GJ $1161 \mathrm{~B}$ & 11.91 & 1.60 & & 21.6 & 220 & 0.050 & 0.021 & 7 & 77 & -0.86 & 0.30 & 18.9 & 10.6 \\
\hline Gl 479 & 10.67 & 1.54 & M3 & 9.7 & 312 & 0.132 & 0.023 & 59 & 7 & -0.55 & 0.14 & 71.4 & 8.0 \\
\hline Gl 479.1 & 6.96 & 0.59 & $\mathrm{dG} 2 \mathrm{e}$ & 35.8 & 488 & 0.107 & 0.016 & 74 & 8 & -0.13 & 0.16 & 81.5 & 125.2 \\
\hline G123-049 & 12.14 & & $\mathrm{~m}$ & 25.6 & 439 & 0.103 & 0.020 & 53 & 11 & -0.33 & 0.15 & 67.4 & 52.7 \\
\hline LTT 13593 & 14.81 & 1.63 & $\mathrm{~m}$ & 21.1 & 467 & 0.025 & 0.010 & 8 & 5 & -0.61 & 0.26 & 12.5 & 6.7 \\
\hline Gl $482 \mathrm{~A}$ & 3.46 & 0.36 & $\mathrm{~F} 0 \mathrm{~V}$ & 11.8 & 146 & 2.039 & 0.121 & 888 & 11 & -0.11 & 0.06 & 1575.5 & 264.0 \\
\hline Gl 482 B & 3.52 & & Fo V & 11.8 & 146 & 2.039 & 0.121 & 888 & 11 & -0.11 & 0.06 & 1575.5 & 264.0 \\
\hline HD 110463 & 8.29 & 0.95 & K3 V & 23.2 & 348 & 0.061 & 0.016 & 28 & 8 & -0.27 & 0.23 & 41.9 & 27.0 \\
\hline Gl 483 & 7.04 & 0.94 & K3 V & 15.1 & 320 & 0.158 & 0.025 & 82 & 11 & -0.52 & 0.13 & 88.0 & 23.9 \\
\hline GJ 1165 & 7.94 & 0.96 & $\mathrm{~K} 2 \mathrm{~V}$ & 21.2 & 324 & 0.081 & 0.019 & 29 & 10 & -0.42 & 0.19 & 49.3 & 26.5 \\
\hline WD1246+586 & 15.56 & & DC8 : & 18.5 & 352 & 0.516 & 0.041 & 418 & 20 & -0.42 & 0.07 & 313.8 & 128.7 \\
\hline Gl 486.1 & 6.31 & 0.70 & G7 V & 17.2 & 424 & 0.133 & 0.021 & 77 & 14 & -0.55 & 0.12 & 71.9 & 25.4 \\
\hline Wo 9417 & 5.85 & 0.46 & F6 V & 24.2 & 479 & 0.503 & 0.034 & 565 & 5 & -0.19 & 0.06 & 367.7 & 256.7 \\
\hline Gl 487 & 10.90 & 1.64 & M3 & 10.2 & 478 & 0.226 & 0.025 & 159 & 8 & -0.22 & 0.10 & 161.8 & 20.1 \\
\hline Gl 488 & 8.49 & 1.40 & $\mathrm{M} 0.5 \mathrm{Ve}$ & 10.8 & 221 & 0.061 & 0.021 & 12 & 36 & -0.29 & 0.31 & 41.5 & 5.8 \\
\hline Wo 9421 & 6.11 & 0.50 & F5 V & 32.5 & 341 & 0.135 & 0.023 & 61 & 9 & -0.40 & 0.14 & 83.8 & 105.6 \\
\hline Gl 488.1 & 4.26 & 0.21 & A7 III & 47.6 & 333 & 0.021 & 0.010 & 8 & 19 & -0.44 & 0.42 & 12.3 & 33.4 \\
\hline Gl $490 \mathrm{~A}$ & 10.68 & 1.44 & M0 Ve & 18.1 & 521 & 0.838 & 0.042 & 999 & 3 & -0.22 & 0.05 & 598.5 & 234.1 \\
\hline Gl $490 \mathrm{~B}$ & 13.20 & 1.64 & $\mathrm{dM} 4 \mathrm{e}$ & 18.1 & 521 & 0.838 & 0.042 & 999 & 18 & -0.22 & 0.05 & 598.5 & 234.1 \\
\hline Gl 493.1 & 13.40 & 1.75 & dM5 e & 8.1 & 272 & 0.140 & 0.025 & 68 & 5 & -0.16 & 0.18 & 104.5 & 8.2 \\
\hline *LTT 4974 & 10.95 & & $\mathrm{dM} 2$ & 16.8 & 638 & 0.010 & 0.004 & 11 & 14 & -0.75 & 0.80 & 4.2 & 1.4 \\
\hline Gl 494 & 9.75 & 1.47 & $\mathrm{dM} 1.5 \mathrm{e}$ & 11.4 & 177 & 1.566 & 0.099 & 705 & 10 & -0.01 & 0.06 & 1293.0 & 202.1 \\
\hline Gl 498 & 9.30 & 1.17 & $\mathrm{dK} 8$ & 21.9 & 516 & 0.023 & 0.009 & 8 & 12 & -0.35 & 0.30 & 15.0 & 8.6 \\
\hline Gl 499 A & 9.44 & 1.29 & dM0 & 18.6 & 493 & 0.151 & 0.019 & 130 & 7 & -0.40 & 0.12 & 93.4 & 38.6 \\
\hline Gl 499 B & 14.90 & & & 18.6 & 493 & 0.151 & 0.019 & 130 & 7 & -0.40 & 0.12 & 93.4 & 38.6 \\
\hline GJ 1167 A & 14.18 & 1.72 & dM5 & 11.5 & 508 & 0.098 & 0.016 & 76 & 3 & -0.12 & 0.16 & 75.0 & 12.0 \\
\hline Gl 501 A & 4.98 & 0.45 & F5 V & 18.3 & 244 & 0.876 & 0.063 & 538 & 9 & -0.38 & 0.06 & 551.7 & 221.4 \\
\hline Gl 501 B & 5.17 & & F5 V & 18.3 & 244 & 0.876 & 0.063 & 538 & 9 & -0.38 & 0.06 & 551.7 & 221.4 \\
\hline Gl 501.1 & 8.16 & 0.58 & F5 IV & 107.5 & 506 & 0.886 & 0.044 & 999 & 6 & 0.04 & 0.05 & 755.2 & 10447.1 \\
\hline Gl 501.2 & 4.85 & 0.70 & G3 V & 20.5 & 282 & 0.100 & 0.022 & 34 & 9 & -0.70 & 0.15 & 45.9 & 23.1 \\
\hline Gl 502 & 4.26 & 0.57 & G0 V & 9.2 & 504 & 0.255 & 0.037 & 78 & 10 & -0.73 & 0.09 & 113.2 & 11.4 \\
\hline Gl 503.2 & 6.83 & 0.60 & G1 V & 25.7 & 634 & 0.176 & 0.019 & 195 & 4 & -0.36 & 0.09 & 112.7 & 89.1 \\
\hline Gl 504 & 5.20 & 0.58 & G0 V & 18.0 & 331 & 1.122 & 0.087 & 415 & 9 & -0.14 & 0.08 & 849.1 & 327.5 \\
\hline Gl 505 A & 6.59 & 0.94 & $\mathrm{~K} 1 \mathrm{~V}$ & 11.2 & 279 & 0.143 & 0.027 & 48 & 13 & -0.65 & 0.13 & 69.5 & 10.5 \\
\hline Gl 505 B & 9.60 & & M1 V & 11.2 & 279 & 0.143 & 0.027 & 48 & 13 & -0.65 & 0.13 & 69.5 & 10.5 \\
\hline HD 115781 & 8.13 & 1.14 & $\mathrm{~K} 1 \mathrm{~V}$ & 416.7 & 477 & 0.071 & 0.015 & 40 & 33 & 0.29 & 0.22 & 70.3 & 14603.9 \\
\hline Gl 508 A & 8.94 & 1.48 & $\mathrm{dM} 1.5 \mathrm{e}$ & 10.4 & 554 & 0.050 & 0.012 & 32 & 12 & -0.44 & 0.20 & 29.8 & 3.8 \\
\hline Gl 508 B & 9.80 & & & 10.4 & 554 & 0.050 & 0.012 & 32 & 12 & -0.44 & 0.20 & 29.8 & 3.8 \\
\hline LHS 2729 & 12.90 & & $\mathrm{~m}$ & 13.4 & 309 & 0.137 & 0.024 & 56 & 4 & -0.31 & 0.16 & 91.0 & 19.5 \\
\hline Gl 509 A & 9.52 & 1.33 & dM0 & 18.5 & 428 & 0.026 & 0.010 & 11 & 23 & -1.00 & 0.35 & 7.9 & 3.2 \\
\hline Gl 509 B & 9.80 & & $\mathrm{dK} 6$ & 18.5 & 428 & 0.026 & 0.010 & 11 & 23 & -1.00 & 0.35 & 7.9 & 3.2 \\
\hline Gl 509.1 & 9.75 & 1.26 & M0 V & 25.1 & 697 & 0.198 & 0.019 & 222 & 11 & -0.36 & 0.08 & 126.9 & 95.8 \\
\hline HD 116656 & 2.25 & 0.02 & $\mathrm{dA} 1 \mathrm{p}$ & 24.0 & 545 & 0.019 & 0.008 & 8 & 57 & -0.34 & 0.36 & 12.5 & 8.6 \\
\hline HD 116657 & 3.95 & 0.13 & $\mathrm{~A} 1 \mathrm{~m}$ & 24.0 & 545 & 0.019 & 0.008 & 8 & 38 & -0.34 & 0.36 & 12.5 & 8.6 \\
\hline HD 116842 & 4.01 & 0.16 & A5 V & 24.9 & 552 & 0.026 & 0.010 & 13 & 2 & 0.24 & 0.34 & 25.4 & 18.8 \\
\hline LHS 2739 & 13.60 & & M4 & & 300 & 0.099 & 0.021 & 32 & 4 & 0.00 & 0.22 & 82.2 & \\
\hline Gl 514 & 9.05 & 1.50 & M1 V & 7.6 & 311 & 0.058 & 0.016 & 21 & 10 & -0.40 & 0.26 & 35.8 & 2.5 \\
\hline G165-008 & 11.95 & 1.57 & $\mathrm{M} 4 \mathrm{e}$ & 7.5 & 450 & 0.410 & 0.032 & 335 & 6 & -0.33 & 0.07 & 269.1 & 17.9 \\
\hline Gl 516 A & 12.01 & 1.53 & dM3.5e & 16.3 & 340 & 0.076 & 0.018 & 32 & 23 & -0.48 & 0.18 & 43.6 & 13.8 \\
\hline Gl 516 B & 12.30 & & dM3.5e & 16.3 & 340 & 0.076 & 0.018 & 32 & 23 & -0.48 & 0.18 & 43.6 & 13.8 \\
\hline GJ 2102 & 7.98 & 1.00 & K0 & 18.5 & 353 & 0.046 & 0.015 & 16 & 24 & -0.36 & 0.26 & 29.5 & 12.0 \\
\hline Gl 517 & 9.31 & 1.21 & K5 & 19.8 & 325 & 1.061 & 0.060 & 793 & 6 & -0.21 & 0.05 & 763.6 & 358.2 \\
\hline HD 118098 & 3.37 & 0.11 & A3 V & 22.5 & 252 & 0.025 & 0.012 & 10 & 15 & -0.26 & 0.45 & 17.5 & 10.6 \\
\hline Gl 519 & 9.04 & 1.42 & $\mathrm{dM} 1$ & 10.9 & 578 & 0.029 & 0.010 & 12 & 29 & -0.57 & 0.21 & 15.4 & 2.2 \\
\hline Gl $520 \mathrm{~A}$ & 10.17 & 1.40 & $\mathrm{dM} 0.5$ & 21.9 & 557 & 0.030 & 0.011 & 10 & 14 & -0.74 & 0.21 & 13.3 & 7.6 \\
\hline Gl 520 B & 11.00 & & & 21.9 & 557 & 0.030 & 0.011 & 10 & 14 & -0.74 & 0.21 & 13.3 & 7.6 \\
\hline GJ 1175 & 6.98 & 0.86 & $\mathrm{~K} 1 \mathrm{~V}$ & 15.6 & 304 & 0.191 & 0.028 & 72 & 21 & -0.33 & 0.14 & 125.6 & 36.6 \\
\hline Gl $521.2 \mathrm{~A}$ & 6.33 & 0.54 & F7 V & 25.3 & 541 & 0.365 & 0.029 & 372 & 4 & -0.15 & 0.08 & 274.5 & 209.4 \\
\hline
\end{tabular}




\begin{tabular}{|c|c|c|c|c|c|c|c|c|c|c|c|c|c|}
\hline Name & $V$ & $B-V$ & Sp. type & $d(\mathrm{pc})$ & Exp. & $C R$ & $\pm C R$ & $\mathrm{Li}$ & $\Delta$ & $h r$ & $\Delta h r$ & $f_{\mathrm{x}-14}$ & $L_{\mathrm{x} 27}$ \\
\hline Gl $521.2 \mathrm{~B}$ & 10.46 & 1.36 & & 25.3 & 541 & 0.365 & 0.029 & 372 & 21 & -0.15 & 0.08 & 274.5 & 209.4 \\
\hline LHS 2783 & 13.50 & & $\mathrm{~m}$ & 13.0 & 338 & 0.060 & 0.016 & 26 & 6 & -0.78 & 0.19 & 25.1 & 5.1 \\
\hline Gl 525.1 & 4.23 & 0.38 & F3 IV & 19.3 & 283 & 0.615 & 0.049 & 404 & 6 & 0.02 & 0.08 & 517.9 & 230.0 \\
\hline GJ 1177 A & 8.94 & 1.32 & K5 V & 16.4 & 271 & 0.115 & 0.025 & 27 & 11 & -0.52 & 0.18 & 63.9 & 20.5 \\
\hline GJ 1177 B & 9.12 & 1.36 & & 16.4 & 271 & 0.115 & 0.025 & 27 & 10 & -0.52 & 0.18 & 63.9 & 20.5 \\
\hline Gl $527 \mathrm{~A}$ & 4.50 & 0.48 & F7 V & 15.6 & 376 & 0.563 & 0.061 & 179 & 11 & -0.54 & 0.09 & 306.5 & 89.3 \\
\hline Gl $527 \mathrm{~B}$ & 11.00 & & M2 & 15.6 & 376 & 0.563 & 0.061 & 179 & 11 & -0.54 & 0.09 & 306.5 & 89.3 \\
\hline LTT 14031 & 14.34 & & $\mathrm{~m}$ & 14.6 & 330 & 0.056 & 0.016 & 26 & 28 & 0.08 & 0.27 & 49.3 & 12.7 \\
\hline Gl 528 A & 7.61 & 1.12 & $\mathrm{~K} 4 \mathrm{~V}$ & 13.6 & 453 & 0.127 & 0.020 & 87 & 3 & -0.44 & 0.12 & 76.2 & 17.0 \\
\hline Gl $528 \mathrm{~B}$ & 8.03 & & dK6 & 13.6 & 453 & 0.127 & 0.020 & 87 & 3 & -0.44 & 0.12 & 76.2 & 17.0 \\
\hline Gl 530 & 6.44 & 0.69 & G5 V & 19.9 & 217 & 0.063 & 0.020 & 16 & 5 & -0.74 & 0.26 & 27.6 & 13.1 \\
\hline *Gl 531 & 7.38 & 0.90 & $\mathrm{~K} 1 \mathrm{~V}$ & 16.4 & 426 & 0.146 & 0.019 & 94 & 15 & -0.19 & 0.10 & 106.3 & 34.3 \\
\hline G150-046 & 11.63 & 1.50 & M2 & 15.8 & 361 & 0.029 & 0.011 & 10 & 21 & -1.00 & 9.99 & 8.8 & 2.6 \\
\hline Gl 534 & 2.68 & 0.58 & G0 IV & 11.3 & 377 & 0.144 & 0.023 & 55 & 13 & -0.65 & 0.13 & 70.0 & 10.8 \\
\hline LHS 2836 & 13.00 & & $\mathrm{~K} 2:$ & 9.5 & 313 & 0.195 & 0.027 & 113 & 8 & -0.33 & 0.13 & 127.7 & 13.8 \\
\hline Gl 536 & 9.70 & 1.46 & M1 & 10.2 & 398 & 0.033 & 0.011 & 11 & 67 & -0.83 & 0.27 & 12.9 & 1.6 \\
\hline $135954+1355.9$ & 10.65 & 1.47 & dM0.5 & 19.5 & 296 & 0.025 & 0.011 & 8 & 9 & -0.65 & 0.31 & 12.1 & 5.5 \\
\hline LHS 2852 & 12.15 & 1.70 & M0.5 & & 286 & 0.153 & 0.026 & 72 & 6 & -0.43 & 0.14 & 92.3 & \\
\hline Gl $537 \mathrm{~A}$ & 9.85 & 1.48 & dM3 e & 11.3 & 390 & 0.083 & 0.018 & 41 & 5 & -0.46 & 0.17 & 48.6 & 7.4 \\
\hline Gl 537 B & 9.95 & & $\mathrm{dM} 3 \mathrm{e}$ & 11.3 & 390 & 0.083 & 0.018 & 41 & 5 & -0.46 & 0.17 & 48.6 & 7.4 \\
\hline HD 124106 & 7.93 & 0.86 & K1 V & 23.1 & 294 & 0.032 & 0.012 & 14 & 8 & -1.00 & 1.03 & 9.6 & 6.1 \\
\hline Gl 540.2 & 13.86 & 1.53 & $\mathrm{dM} 5.5 \mathrm{e}$ & 12.4 & 282 & 0.199 & 0.029 & 142 & 9 & -0.17 & 0.13 & 147.4 & 27.3 \\
\hline HD 124498 & 10.40 & 1.28 & $\mathrm{~K} 4 \mathrm{~V}$ & 26.5 & 308 & 0.780 & 0.052 & 665 & 16 & -0.19 & 0.07 & 570.0 & 477.3 \\
\hline LTT 5581 & 13.96 & 1.60 & $\mathrm{~m}$ & 26.5 & 308 & 0.780 & 0.052 & 665 & 50 & -0.19 & 0.07 & 570.0 & 477.3 \\
\hline HD 124642 & 8.04 & 1.08 & K4 & 17.3 & 479 & 0.052 & 0.013 & 24 & 19 & -0.43 & 0.23 & 31.5 & 11.3 \\
\hline *Gl 540.3 & 6.31 & 0.60 & G4 V & 21.0 & 522 & 0.205 & 0.020 & 156 & 6 & -0.38 & 0.10 & 129.2 & 68.5 \\
\hline Wo 9473 & 4.08 & 0.52 & F6 III & 21.4 & 339 & 0.946 & 0.078 & 395 & 8 & -0.03 & 0.08 & 771.2 & 423.1 \\
\hline Wo $9474 \mathrm{~A}$ & 4.75 & 0.20 & $\mathrm{~A} 7 \mathrm{~V}$ & 29.9 & 686 & 0.058 & 0.012 & 41 & 43 & -0.47 & 0.15 & 33.9 & 36.1 \\
\hline Wo $9474 \mathrm{~B}$ & 8.23 & 0.82 & $\mathrm{~K} 1 \mathrm{~V}$ & 29.9 & 686 & 0.058 & 0.012 & 41 & 7 & -0.47 & 0.15 & 33.9 & 36.1 \\
\hline G165-061 & 13.10 & & $\mathrm{~m}$ & 16.1 & 484 & 0.114 & 0.018 & 75 & 8 & -0.45 & 0.13 & 67.8 & 21.0 \\
\hline Gl 541.2 & 10.25 & 1.44 & $\mathrm{dM} 0 \mathrm{p}$ & 18.7 & 892 & 0.089 & 0.012 & 85 & 61 & -0.28 & 0.12 & 61.1 & 25.4 \\
\hline Gl 542.2 & 9.10 & 1.30 & K5 & 21.5 & 329 & 0.031 & 0.012 & 10 & 27 & -0.34 & 0.36 & 20.2 & 11.2 \\
\hline HD 125451 & 5.41 & 0.38 & $\mathrm{dF} 1$ & 26.1 & 234 & 0.202 & 0.033 & 70 & 11 & -0.14 & 0.16 & 152.6 & 124.4 \\
\hline Steph 1145 & 12.31 & 1.51 & M0:e: & 20.3 & 614 & 0.159 & 0.018 & 148 & 10 & -0.13 & 0.11 & 121.4 & 59.8 \\
\hline Gl 546 & 8.55 & 1.26 & K8 V & 14.4 & 556 & 0.059 & 0.013 & 29 & 16 & -0.29 & 0.18 & 40.1 & 10.0 \\
\hline Gl $548 \mathrm{~A}$ & 9.75 & 1.44 & dM1 & 16.8 & 425 & 0.045 & 0.012 & 26 & 36 & -0.12 & 0.26 & 34.8 & 11.8 \\
\hline Gl 548 B & 10.00 & 1.46 & $\mathrm{dM} 2$ & 15.8 & 425 & 0.045 & 0.012 & 26 & 7 & -0.12 & 0.26 & 34.8 & 10.4 \\
\hline Gl 549 A & 4.06 & 0.50 & F7 V & 14.6 & 724 & 1.996 & 0.055 & 999 & 4 & 0.02 & 0.03 & 1679.8 & 427.1 \\
\hline Gl 549 B & 11.50 & 1.50 & M3 & 14.6 & 724 & 1.996 & 0.055 & 999 & 74 & 0.02 & 0.03 & 1679.8 & 427.1 \\
\hline WD $1424+240$ & 15.41 & & DC8 & 17.2 & 384 & 0.343 & 0.032 & 305 & 12 & 0.03 & 0.10 & 290.9 & 103.5 \\
\hline GJ 1183 A & 13.95 & 1.65 & $\mathrm{~m}$ & 16.2 & 331 & 0.131 & 0.022 & 68 & 23 & -0.17 & 0.16 & 96.7 & 30.4 \\
\hline GJ 1183 B & 14.03 & 1.68 & $\mathrm{~m}$ & 16.2 & 331 & 0.131 & 0.022 & 68 & 9 & -0.17 & 0.16 & 96.7 & 30.4 \\
\hline Gl $550.2 \mathrm{~A}$ & 4.83 & 0.72 & G2 IV & 41.5 & 247 & 1.211 & 0.071 & 999 & 8 & 0.07 & 0.06 & 1051.3 & 2165.6 \\
\hline Gl $550.2 \mathrm{~B}$ & 9.00 & & G4 V & 41.5 & 247 & 1.211 & 0.071 & 999 & 8 & 0.07 & 0.06 & 1051.3 & 2165.6 \\
\hline Gl 551 & 11.05 & 1.83 & dM5 e & 1.3 & 374 & 1.374 & 0.111 & 445 & 3 & -0.41 & 0.07 & 843.2 & 1.7 \\
\hline HD 127068 & 8.38 & & G5 & 102.0 & 466 & 0.401 & 0.032 & 347 & 7 & 0.20 & 0.08 & 375.5 & 4678.5 \\
\hline Gl 554 & 8.70 & 1.04 & dK5 & 22.0 & 625 & 0.019 & 0.007 & 9 & 11 & -0.74 & 0.25 & 8.2 & 4.8 \\
\hline LP $440-38$ & 13.61 & & $\mathrm{~m}$ & 16.7 & 361 & 0.047 & 0.015 & 19 & 18 & -0.52 & 0.22 & 25.9 & 8.6 \\
\hline HD 128642 & 6.91 & 0.76 & G5 & 19.6 & 747 & 0.032 & 0.009 & 11 & 13 & -1.00 & 0.17 & 9.8 & 4.5 \\
\hline Gl 556 & 7.23 & 0.99 & K3 V & 13.4 & 775 & 0.040 & 0.010 & 20 & 33 & -0.86 & 0.11 & 15.1 & 3.3 \\
\hline Gl 557 & 4.46 & 0.36 & $\mathrm{~F} 2 \mathrm{~V}$ & 15.5 & 457 & 0.171 & 0.022 & 112 & 2 & -0.32 & 0.12 & 112.8 & 32.2 \\
\hline HD 128311 & 7.50 & 0.99 & K0 & 16.6 & 345 & 0.149 & 0.024 & 88 & 9 & -0.29 & 0.14 & 100.8 & 33.2 \\
\hline G223-074 & 11.74 & & $\mathrm{~m}$ & 14.7 & 627 & 0.122 & 0.017 & 95 & 6 & -0.31 & 0.12 & 81.7 & 21.1 \\
\hline Gl 559 A & 0.01 & 0.64 & $\mathrm{G} 2 \mathrm{~V}$ & 1.3 & 420 & 3.177 & 0.212 & 589 & 7 & -0.98 & 0.02 & 990.0 & 2.2 \\
\hline Gl 559 B & 1.34 & 0.84 & K0 V & 1.3 & 420 & 3.177 & 0.212 & 589 & 7 & -0.98 & 0.02 & 990.0 & 2.2 \\
\hline HD 128400 & 6.73 & & G5 V & 20.3 & 272 & 0.140 & 0.026 & 57 & 11 & -0.50 & 0.16 & 79.4 & 39.2 \\
\hline Gl 559.1 & 7.54 & 0.61 & dG0 e & 33.9 & 582 & 0.981 & 0.042 & 999 & 1 & -0.14 & 0.04 & 742.4 & 1020.7 \\
\hline Wo 9491 & 3.88 & 0.38 & F2 III & 18.7 & 337 & 0.642 & 0.046 & 588 & 3 & 0.17 & 0.07 & 591.5 & 247.3 \\
\hline Wo 9492 & 10.83 & 1.55 & M3 & 10.8 & 1069 & 0.022 & 0.006 & 18 & 25 & -0.05 & 0.25 & 17.7 & 2.5 \\
\hline$* \mathrm{Gl} 561.1 \mathrm{~A}$ & 5.10 & 0.34 & F2 III-IV & 30.4 & 454 & 0.114 & 0.016 & 35 & 30 & -0.22 & 0.12 & 81.7 & 90.3 \\
\hline$* \mathrm{Gl} 561.1 \mathrm{~B}$ & 7.14 & & dF9 & 30.4 & 454 & 0.114 & 0.016 & 35 & 15 & -0.22 & 0.12 & 81.7 & 90.3 \\
\hline HD 130307 & 7.78 & 0.90 & G8 V & 19.7 & 350 & 0.031 & 0.012 & 8 & 7 & -0.11 & 0.35 & 24.0 & 11.1 \\
\hline Gl 563.4 & 5.15 & 0.41 & F5 IV-V & 23.6 & 379 & 0.283 & 0.031 & 189 & 11 & -0.30 & 0.10 & 190.0 & 127.1 \\
\hline Gl 564 & 5.85 & 0.56 & $\mathrm{G} 2 \mathrm{~V}$ & 18.0 & 529 & 0.407 & 0.031 & 427 & 13 & -0.35 & 0.07 & 262.5 & 101.2 \\
\hline HD 131023 & 7.40 & 0.76 & $\mathrm{dG} 5$ & 27.2 & 330 & 0.089 & 0.021 & 26 & 6 & -0.32 & 0.21 & 58.9 & 52.3 \\
\hline LTT 14398 & 8.90 & & $\mathrm{k}$ & 27.2 & 330 & 0.089 & 0.021 & 26 & 1 & -0.32 & 0.21 & 58.9 & 52.3 \\
\hline *Gl 565 & 7.83 & 1.00 & K5 V & 17.0 & 1207 & 0.024 & 0.004 & 47 & 7 & -0.78 & 0.11 & 9.9 & 3.4 \\
\hline Gl 566 A & 4.70 & 0.73 & G8 Ve & 6.7 & 400 & 2.440 & 0.183 & 416 & 6 & -0.31 & 0.07 & 1626.7 & 87.3 \\
\hline Gl $566 \mathrm{~B}$ & 6.97 & 1.16 & $\mathrm{~K} 4 \mathrm{Ve}$ & 6.7 & 400 & 2.440 & 0.183 & 416 & 6 & -0.31 & 0.07 & 1626.7 & 87.3 \\
\hline Gl 567 & 6.02 & 0.84 & $\mathrm{~K} 2 \mathrm{~V}$ & 11.5 & 398 & 0.363 & 0.034 & 245 & 14 & -0.48 & 0.08 & 209.2 & 33.3 \\
\hline Gl $568 \mathrm{~A}$ & 12.19 & 1.61 & M3.5 & 10.2 & 451 & 0.040 & 0.013 & 17 & 17 & -0.45 & 0.22 & 23.6 & 2.9 \\
\hline Gl 568 B & 12.70 & & & 10.2 & 451 & 0.040 & 0.013 & 17 & 17 & -0.45 & 0.22 & 23.6 & 2.9 \\
\hline Gl 569 AB & 10.20 & 1.48 & dM0 e & 9.8 & 385 & 0.489 & 0.038 & 361 & 5 & -0.41 & 0.07 & 300.3 & 34.6 \\
\hline LP 222-015 & 14.67 & & $\mathrm{~m}$ & 23.8 & 655 & 0.020 & 0.008 & 7 & 26 & 0.23 & 0.40 & 18.6 & 12.6 \\
\hline HD 133002 & 5.64 & 0.68 & F9 V & 43.3 & 753 & 0.115 & 0.014 & 146 & 9 & -0.20 & 0.12 & 83.7 & 187.8 \\
\hline LHS 3001 & 15.50 & & M5 & 21.6 & 389 & 0.015 & 0.007 & 9 & 68 & -0.11 & 0.42 & 11.8 & 6.6 \\
\hline LHS 3002 & 18.60 & & M7 : & 21.6 & 389 & 0.015 & 0.007 & 9 & 83 & -0.11 & 0.42 & 11.8 & 6.6 \\
\hline$* \mathrm{Gl} 570 \mathrm{~A}$ & 5.75 & 1.10 & $\mathrm{~K} 5 \mathrm{Ve}$ & 5.9 & 820 & 0.200 & 0.016 & 378 & 6 & -0.77 & 0.06 & 84.5 & 3.5 \\
\hline * Gl $570 \mathrm{~B}$ & 8.00 & 1.50 & $\mathrm{M} 2 \mathrm{~V}$ & 5.9 & 820 & 0.200 & 0.016 & 378 & 27 & -0.77 & 0.06 & 84.5 & 3.5 \\
\hline Wo 9503 & 4.49 & 0.32 & F0 V & 27.9 & 406 & 0.023 & 0.010 & 8 & 23 & -0.70 & 0.35 & 10.5 & 9.8 \\
\hline
\end{tabular}




\begin{tabular}{|c|c|c|c|c|c|c|c|c|c|c|c|c|c|}
\hline Name & $V$ & $B-V$ & Sp. type & $d(\mathrm{pc})$ & Exp. & $C R$ & $\pm C R$ & $\mathrm{Li}$ & $\Delta$ & $h r$ & $\Delta h r$ & $f_{\mathrm{x}-14}$ & $L_{\mathrm{x} 27}$ \\
\hline Gl 570.1 & 6.35 & 0.71 & G5 V & 24.5 & 349 & 0.017 & 0.008 & 8 & 29 & & & 14.0 & 10.1 \\
\hline HD 132254 & 5.63 & 0.50 & $\mathrm{~F} 8 \mathrm{~V}$ & 24.8 & 740 & 0.027 & 0.009 & 9 & 29 & -0.10 & 0.28 & 20.7 & 15.2 \\
\hline Gl 572 & 9.13 & 1.43 & dMo & 11.7 & 775 & 0.147 & 0.016 & 172 & 13 & -0.41 & 0.09 & 90.1 & 14.7 \\
\hline Gl 575 A & 5.19 & 0.65 & F9 V n & 12.8 & 612 & 3.414 & 0.076 & 999 & 12 & -0.16 & 0.02 & 2547.5 & 495.9 \\
\hline Gl 575 B & 5.96 & & $\mathrm{dG} 2$ & 12.8 & 612 & 3.414 & 0.076 & 999 & 12 & -0.16 & 0.02 & 2547.5 & 495.9 \\
\hline Gl 577 & 8.42 & 0.68 & dG5 e & 44.2 & 876 & 0.103 & 0.013 & 123 & 7 & -0.09 & 0.11 & 81.0 & 189.7 \\
\hline Gl 578 & 4.93 & 0.43 & F5 V & 19.7 & 380 & 0.208 & 0.026 & 149 & 2 & -0.48 & 0.10 & 120.2 & 55.9 \\
\hline G179-020 & 13.35 & & $\mathrm{~m}$ & 13.5 & 589 & 0.047 & 0.011 & 33 & 19 & -0.46 & 0.18 & 27.5 & 6.0 \\
\hline HD 136351 & 5.00 & 0.50 & $\mathrm{~F} 8 \mathrm{~V}$ & 34.1 & 392 & 0.156 & 0.022 & 110 & 0 & -0.10 & 0.14 & 121.1 & 168.8 \\
\hline GJ $2112 \mathrm{AB}$ & 13.28 & 1.70 & M3 : & & 378 & 0.034 & 0.011 & 18 & 10 & 0.44 & 0.33 & 36.6 & \\
\hline Wo 9520 & 10.11 & 1.51 & dM0 e & 11.4 & 179 & 0.994 & 0.080 & 427 & 16 & -0.24 & 0.08 & 699.9 & 108.6 \\
\hline Gl $584 \mathrm{~A}$ & 5.62 & 0.58 & G2 V & 18.6 & 376 & 0.148 & 0.024 & 76 & 4 & -0.78 & 0.10 & 61.7 & 25.6 \\
\hline Gl 584 B & 5.96 & & G2 V & 18.6 & 376 & 0.148 & 0.024 & 76 & 4 & -0.78 & 0.10 & 61.7 & 25.6 \\
\hline Gl $586 \mathrm{~A}$ & 6.92 & 0.81 & $\mathrm{~K} 2 \mathrm{~V}$ & 19.9 & 380 & 0.146 & 0.023 & 82 & 57 & -0.41 & 0.14 & 89.4 & 42.3 \\
\hline Gl 586 B & 7.58 & 0.92 & $\mathrm{~K} 2 \mathrm{~V}$ & 20.8 & 380 & 0.146 & 0.023 & 82 & 8 & -0.41 & 0.14 & 89.4 & 46.2 \\
\hline Gl 588 & 9.31 & 1.52 & M3 & 5.9 & 416 & 0.040 & 0.013 & 13 & 22 & -1.00 & 0.16 & 12.0 & 0.5 \\
\hline Gl 593 A & 7.43 & 0.91 & $\mathrm{~K} 2 \mathrm{~V}$ & 21.8 & 420 & 0.046 & 0.014 & 14 & 18 & -0.39 & 0.24 & 28.7 & 16.3 \\
\hline Gl 593 B & 7.60 & & & 21.8 & 420 & 0.046 & 0.014 & 14 & 18 & -0.39 & 0.24 & 28.7 & 16.3 \\
\hline Gl 594 & 4.64 & 0.40 & F5 IV-V & 17.5 & 420 & 0.523 & 0.037 & 476 & 14 & -0.29 & 0.07 & 354.3 & 130.0 \\
\hline Gl 596.1A & 5.86 & 0.68 & G5 V & 14.7 & 492 & 0.129 & 0.018 & 96 & 3 & -0.65 & 0.11 & 62.7 & 16.1 \\
\hline Gl 596.1B & 12.00 & & & 14.7 & 492 & 0.129 & 0.018 & 96 & 3 & -0.65 & 0.11 & 62.7 & 16.1 \\
\hline Gl 598 & 4.43 & 0.60 & G0 V & 11.8 & 503 & 0.062 & 0.014 & 30 & 19 & -0.72 & 0.13 & 27.9 & 4.6 \\
\hline Gl 599 A & 6.01 & 0.72 & G6 V & 15.2 & 394 & 0.147 & 0.023 & 67 & 4 & -0.73 & 0.11 & 65.2 & 18.1 \\
\hline Gl 599 B & 12.78 & 0.33 & DA7 & 15.2 & 394 & 0.147 & 0.023 & 67 & 4 & -0.73 & 0.11 & 65.2 & 18.1 \\
\hline HD 141272 & 7.43 & 0.81 & G8 V & 21.4 & 490 & 0.187 & 0.022 & 149 & 3 & -0.30 & 0.12 & 125.5 & 68.6 \\
\hline G179-057 & 13.16 & 1.64 & $\mathrm{~m}$ & 17.0 & 271 & 0.023 & 0.012 & 8 & 21 & -0.58 & 0.29 & 12.0 & 4.2 \\
\hline Gl 600 & 9.36 & 1.42 & dM0 & 22.6 & 540 & 0.022 & 0.008 & 9 & 12 & -0.72 & 0.32 & 9.9 & 6.1 \\
\hline Gl $601 \mathrm{~A}$ & 2.84 & 0.29 & F2 IV & 12.3 & 365 & 0.144 & 0.025 & 58 & 8 & -0.91 & 0.06 & 50.3 & 9.1 \\
\hline HD 142267 & 6.10 & 0.60 & G1 V & 17.5 & 546 & 0.016 & 0.007 & 7 & 14 & -0.68 & 0.33 & 7.5 & 2.7 \\
\hline G180-011 & 13.68 & & $\mathrm{~m}$ & 11.1 & 325 & 0.155 & 0.025 & 97 & 9 & 0.08 & 0.16 & 135.8 & 20.2 \\
\hline Wo 9531 & 5.45 & 0.33 & F0 IV & 41.5 & 269 & 0.036 & 0.014 & 10 & 27 & -0.80 & 0.45 & 14.8 & 30.4 \\
\hline Gl 603 & 3.85 & 0.48 & F6 V & 11.1 & 560 & 0.035 & 0.012 & 10 & 7 & -0.90 & 0.14 & 12.5 & 1.9 \\
\hline HD 143313 & 8.33 & 1.00 & $\mathrm{~K} 2 \mathrm{~V}$ & 87.7 & 630 & 0.643 & 0.034 & 999 & 9 & 0.07 & 0.05 & 557.8 & 5135.6 \\
\hline Gl 606 & 10.50 & 1.51 & dM0 & 13.9 & 198 & 0.052 & 0.020 & 9 & 28 & -0.73 & 0.38 & 23.2 & 5.4 \\
\hline HD 143333 & 5.47 & 0.52 & $\mathrm{~F} 8 \mathrm{~V}$ & 32.8 & 450 & 0.087 & 0.017 & 49 & 13 & -0.27 & 0.18 & 59.6 & 76.7 \\
\hline Gl 609.1 & 4.01 & 0.52 & F8 IV-V & 20.9 & 1603 & 0.750 & 0.023 & 999 & 1 & -0.09 & 0.03 & 587.5 & 307.6 \\
\hline Wo $9540 \mathrm{~A}$ & 4.84 & 0.45 & F6 IV-V & 23.3 & 458 & 0.253 & 0.025 & 233 & 9 & -0.32 & 0.09 & 167.7 & 108.5 \\
\hline Wo 9540 B & 5.00 & & F6 IV-V & 23.3 & 458 & 0.253 & 0.025 & 233 & 9 & -0.32 & 0.09 & 167.7 & 108.5 \\
\hline Wo $9540 \mathrm{C}$ & 7.30 & 0.75 & G8 V & 23.3 & 458 & 0.253 & 0.025 & 233 & 15 & -0.32 & 0.09 & 167.7 & 108.5 \\
\hline Wo 9541 A & 7.46 & 0.74 & G8 V & 28.3 & 458 & 0.085 & 0.016 & 60 & 14 & -0.64 & 0.14 & 41.9 & 40.2 \\
\hline Wo 9541 B & 8.03 & 0.84 & $\mathrm{~K} 2 \mathrm{~V}$ & 29.5 & 458 & 0.085 & 0.016 & 60 & 14 & -0.64 & 0.14 & 41.9 & 43.6 \\
\hline $160747+5304.4$ & 10.19 & 1.45 & dM0 & 17.1 & 499 & 0.023 & 0.009 & 8 & 23 & -0.04 & 0.33 & 18.5 & 6.5 \\
\hline Gl $615.2 \mathrm{~A}$ & 5.64 & 0.51 & F8 V & 21.7 & 764 & 9.487 & 0.115 & 999 & 6 & 0.07 & 0.01 & 8235.7 & 4636.4 \\
\hline Gl $615.2 \mathrm{~B}$ & 6.72 & & G1 V & 21.7 & 764 & 9.487 & 0.115 & 999 & 6 & 0.07 & 0.01 & 8235.7 & 4636.4 \\
\hline Gl 616.2 & 9.96 & 1.48 & $\mathrm{dM} 1.5 \mathrm{e}$ & 20.7 & 1036 & 0.916 & 0.032 & 999 & 14 & -0.12 & 0.03 & 703.3 & 359.2 \\
\hline Gl 617 A & 8.60 & 1.41 & M0 Ve & 10.7 & 1726 & 0.060 & 0.007 & 91 & 13 & -0.50 & 0.10 & 33.9 & 4.6 \\
\hline Gl 617 B & 10.72 & 1.49 & M2.5 & 10.7 & 1726 & 0.060 & 0.007 & 91 & 53 & -0.50 & 0.10 & 33.9 & 4.7 \\
\hline Gl 618 A & 10.60 & 1.57 & M3 & 8.5 & 368 & 0.029 & 0.011 & 10 & 17 & -1.00 & 0.75 & 8.8 & 0.8 \\
\hline Gl 618 B & 14.15 & 1.79 & M5 & 8.5 & 368 & 0.029 & 0.011 & 10 & 17 & -1.00 & 0.75 & 8.8 & 0.8 \\
\hline Wo 9557 A & 5.46 & 0.40 & F3 IV-V & 26.8 & 818 & 0.640 & 0.030 & 999 & 7 & -0.16 & 0.04 & 477.5 & 410.6 \\
\hline Wo 9557 B & 11.00 & & & 26.8 & 818 & 0.640 & 0.030 & 999 & 7 & -0.16 & 0.04 & 477.5 & 410.6 \\
\hline HD 148048 & 4.95 & 0.37 & F3 V & 29.9 & 912 & 0.030 & 0.007 & 19 & 61 & -0.28 & 0.22 & 20.5 & 21.8 \\
\hline HD 147449 & 4.82 & 0.34 & $\mathrm{~F} 0 \mathrm{~V}$ & 27.3 & 439 & 0.043 & 0.013 & 14 & 11 & -0.52 & 0.31 & 24.1 & 21.5 \\
\hline Gl $620.1 \mathrm{~A}$ & 5.39 & 0.63 & G3/5 V & 12.9 & 349 & 0.654 & 0.045 & 646 & 8 & -0.26 & 0.07 & 453.6 & 89.9 \\
\hline Gl $620.1 B$ & 11.00 & -0.14 & DA2 & 12.8 & 355 & 0.688 & 0.068 & 192 & 16 & -0.97 & 0.03 & 218.2 & 42.9 \\
\hline *Gl 621 & 8.40 & 0.96 & K3 V & 21.5 & 378 & 0.026 & 0.010 & 12 & 22 & -0.89 & 0.16 & 9.2 & 5.1 \\
\hline Gl 624 & 4.91 & 0.55 & G0 V & 12.1 & 166 & 1.027 & 0.081 & 493 & 2 & -0.23 & 0.08 & 728.2 & 127.7 \\
\hline Gl $624.1 \mathrm{~A}$ & 2.74 & 0.91 & G8 III & 26.9 & 1846 & 0.017 & 0.005 & 12 & 12 & -0.81 & 0.12 & 6.7 & 5.8 \\
\hline Gl $624.1 \mathrm{~B}$ & 8.80 & & $\mathrm{~K} 2$ & 26.9 & 1846 & 0.017 & 0.005 & 12 & 12 & -0.81 & 0.12 & 6.7 & 5.8 \\
\hline LHS 3197 & 14.30 & & $\mathrm{~m}$ & 12.1 & 378 & 0.021 & 0.009 & 8 & 13 & -0.66 & 0.28 & 10.0 & 1.7 \\
\hline Gl 625 & 10.12 & 1.61 & dM2 & 6.6 & 724 & 0.019 & 0.007 & 9 & 10 & -0.57 & 0.27 & 9.9 & 0.5 \\
\hline Gl 626.1 & 3.89 & 0.91 & K0 IV & 49.0 & 192 & 0.586 & 0.057 & 321 & 7 & 0.25 & 0.10 & 564.1 & 1621.8 \\
\hline Gl $627 \mathrm{~A}$ & 7.68 & 0.85 & K3 V & 19.5 & 469 & 0.052 & 0.013 & 25 & 4 & -0.68 & 0.18 & 24.2 & 11.1 \\
\hline Gl $627 \mathrm{~B}$ & 7.85 & & K3 V & 19.5 & 469 & 0.052 & 0.013 & 25 & 4 & -0.68 & 0.18 & 24.2 & 11.1 \\
\hline Gl 628 & 10.08 & 1.58 & M3.5 & 4.3 & 554 & 0.047 & 0.011 & 27 & 10 & -0.96 & 0.16 & 15.3 & 0.3 \\
\hline Wo 9566 & 8.85 & 0.88 & K1 & 42.6 & 512 & 0.176 & 0.022 & 145 & 16 & -0.11 & 0.12 & 136.1 & 295.0 \\
\hline Gl $630.1 \mathrm{~A}$ & 12.90 & 1.60 & $\mathrm{dM} 4 \mathrm{e}$ & 14.5 & 956 & 0.177 & 0.015 & 251 & 8 & -0.30 & 0.08 & 118.7 & 29.7 \\
\hline Gl 630.1B & 15.00 & 0.49 & DQ8 & 14.5 & 956 & 0.177 & 0.015 & 251 & 22 & -0.30 & 0.08 & 118.7 & 29.7 \\
\hline LP $275-68$ & 12.95 & & $\mathrm{~m}$ & 12.1 & 590 & 0.169 & 0.019 & 144 & 15 & -0.16 & 0.11 & 126.0 & 21.9 \\
\hline GJ 1204 & 13.80 & 1.65 & $\mathrm{~m}$ & 15.3 & 477 & 0.041 & 0.012 & 11 & 31 & -0.49 & 0.34 & 23.2 & 6.5 \\
\hline Gl 631 & 5.75 & 0.82 & K0 Ve & 9.8 & 603 & 0.258 & 0.032 & 148 & 3 & -0.64 & 0.10 & 126.8 & 14.5 \\
\hline Gl 632 & 7.06 & 0.62 & dG3 & 27.2 & 1096 & 0.123 & 0.012 & 249 & 4 & -0.22 & 0.09 & 87.8 & 78.0 \\
\hline $\mathrm{Gl} 632.2 \mathrm{~A}$ & 10.02 & 1.15 & K7 & 31.3 & 1089 & 0.015 & 0.005 & 11 & 9 & 0.36 & 0.36 & 15.3 & 18.0 \\
\hline Gl $632.2 \mathrm{~B}$ & 13.00 & & DA & 31.3 & 1089 & 0.015 & 0.005 & 11 & 4 & 0.36 & 0.36 & 15.3 & 18.0 \\
\hline LP 625-34 & 13.69 & 1.70 & $\mathrm{~m}$ & 11.2 & 631 & 0.058 & 0.012 & 36 & 16 & -0.37 & 0.19 & 36.8 & 5.6 \\
\hline Gl 635 A & 2.91 & 0.65 & G0 IV & 10.8 & 494 & 0.128 & 0.019 & 77 & 15 & -0.72 & 0.09 & 57.4 & 8.0 \\
\hline Gl 635 B & 5.40 & & Ko V & 10.8 & 494 & 0.128 & 0.019 & 77 & 15 & -0.72 & 0.09 & 57.4 & 8.0 \\
\hline G240-023 & 15.65 & 1.95 & $\mathrm{~m}$ & 13.4 & 2374 & 0.071 & 0.007 & 175 & 11 & -0.13 & 0.08 & 53.7 & 11.5 \\
\hline Gl 638 & 8.11 & 1.37 & $\mathrm{~K} 7 \mathrm{~V}$ & 9.8 & 468 & 0.026 & 0.009 & 11 & 39 & -1.00 & 1.49 & 7.9 & 0.9 \\
\hline Wo 9578 & 4.85 & 0.38 & $\mathrm{~F} 2 \mathrm{~V}$ & 26.7 & 777 & 0.235 & 0.019 & 343 & 9 & -0.03 & 0.08 & 191.5 & 163.8 \\
\hline
\end{tabular}




\begin{tabular}{|c|c|c|c|c|c|c|c|c|c|c|c|c|c|}
\hline Name & $V$ & $B-V$ & Sp. type & $d(\mathrm{pc})$ & Exp. & $C R$ & $\pm C R$ & $\mathrm{Li}$ & $\Delta$ & $h r$ & $\Delta h r$ & $f_{\mathrm{x}-14}$ & $L_{\mathrm{x} 27}$ \\
\hline G169-029 & 14.08 & 1.75 & $\mathrm{~m}$ & 10.0 & 742 & 0.063 & 0.011 & 47 & 10 & -0.35 & 0.15 & 40.5 & 4.9 \\
\hline Gl 641 & 6.64 & 0.76 & G8 V & 16.9 & 491 & 0.317 & 0.028 & 286 & 9 & -0.07 & 0.09 & 251.8 & 86.6 \\
\hline Gl 643 & 11.80 & 1.69 & sdM4 & 6.5 & 445 & 3.968 & 0.245 & 906 & 75 & -0.26 & 0.06 & 2750.6 & 138.8 \\
\hline Gl $644 \mathrm{~A}$ & 9.69 & 1.57 & M3 & 5.7 & 445 & 3.968 & 0.245 & 906 & 10 & -0.26 & 0.06 & 2750.6 & 108.4 \\
\hline Gl 644 B & 9.90 & & & 5.7 & 445 & 3.968 & 0.245 & 906 & 10 & -0.26 & 0.06 & 2750.6 & 108.4 \\
\hline GJ 1207 & 12.28 & 1.60 & dM3.5 & 9.6 & 472 & 0.374 & 0.043 & 180 & 6 & -0.46 & 0.10 & 219.7 & 24.1 \\
\hline Gl 648 & 4.89 & 0.48 & F6 V & 15.1 & 2622 & 0.406 & 0.013 & 999 & 1 & -0.42 & 0.03 & 246.9 & 67.2 \\
\hline Gl $649.1 \mathrm{~A}$ & 7.83 & 0.98 & dK8 & 18.0 & 823 & 0.077 & 0.012 & 63 & 6 & -0.57 & 0.11 & 40.5 & 15.6 \\
\hline Gl $649.1 B$ & 11.19 & 1.47 & & 18.0 & 823 & 0.077 & 0.012 & 63 & 6 & -0.57 & 0.11 & 40.5 & 15.6 \\
\hline HD 153580 & 5.29 & 0.50 & F6 V & 26.3 & 243 & 0.255 & 0.036 & 100 & 10 & -0.26 & 0.14 & 176.7 & 146.4 \\
\hline Wo $9582 \mathrm{~A}$ & 8.65 & 1.04 & K4 V & 25.2 & 1056 & 0.026 & 0.006 & 20 & 23 & -1.00 & 0.13 & 7.8 & 5.9 \\
\hline Wo 9582 B & 10.31 & 1.40 & $\mathrm{~K} 4$ & 24.9 & 1056 & 0.026 & 0.006 & 20 & 12 & -1.00 & 0.13 & 7.8 & 5.8 \\
\hline Gl 654 & 10.08 & 1.44 & M3.5V & 10.6 & 386 & 0.023 & 0.011 & 8 & 65 & -0.33 & 0.40 & 15.0 & 2.0 \\
\hline Gl 654.1 & 6.00 & 0.58 & F9 V & 20.4 & 496 & 0.230 & 0.023 & 193 & 1 & -0.48 & 0.09 & 132.6 & 65.8 \\
\hline Gl 654.2 & 7.09 & 0.94 & K0 V & 227.3 & 539 & 0.012 & 0.006 & 8 & 79 & & & 9.8 & 606.0 \\
\hline Wo $9584 \mathrm{~A}$ & 5.63 & 0.47 & F7 V & 27.0 & 1270 & 0.484 & 0.021 & 999 & 5 & -0.25 & 0.04 & 338.4 & 294.1 \\
\hline Wo 9584 B & 5.73 & & F7 V & 27.0 & 1270 & 0.484 & 0.021 & 999 & 5 & -0.25 & 0.04 & 338.4 & 294.1 \\
\hline Wo $9584 \mathrm{C}$ & 13.50 & & M3 : & 27.0 & 1270 & 0.484 & 0.021 & 999 & 5 & -0.25 & 0.04 & 338.4 & 294.1 \\
\hline Gl 657 & 3.33 & 0.41 & F0 IVn & 21.9 & 304 & 0.108 & 0.021 & 43 & 17 & -0.88 & 0.12 & 39.4 & 22.7 \\
\hline Gl 659 A & 8.85 & 1.16 & dK8 & 21.2 & 1214 & 0.053 & 0.008 & 60 & 9 & -0.51 & 0.11 & 29.7 & 16.0 \\
\hline Gl 659 B & 9.34 & 1.25 & dK8 & 20.9 & 1214 & 0.053 & 0.008 & 60 & 17 & -0.51 & 0.11 & 29.7 & 15.5 \\
\hline HD 155902 & 6.98 & 0.70 & G5 & 28.2 & 1510 & 0.028 & 0.006 & 26 & 15 & -0.81 & 0.11 & 11.1 & 10.6 \\
\hline Gl 661 A & 9.96 & 1.49 & M3 & 6.3 & 1145 & 0.058 & 0.009 & 65 & 22 & -0.68 & 0.11 & 27.3 & 1.3 \\
\hline Gl 661 B & 10.40 & & M3.5 & 6.3 & 1145 & 0.058 & 0.009 & 65 & 22 & -0.68 & 0.11 & 27.3 & 1.3 \\
\hline Gl $663 \mathrm{~A}$ & 5.07 & 0.85 & $\mathrm{~K} 1 \mathrm{Ve}$ & 6.0 & 291 & 0.854 & 0.058 & 664 & 9 & -0.58 & 0.05 & 447.2 & 19.2 \\
\hline Gl 663 B & 5.11 & 0.86 & $\mathrm{~K} 1 \mathrm{Ve}$ & 6.0 & 291 & 0.854 & 0.058 & 664 & 7 & -0.58 & 0.05 & 447.2 & 19.2 \\
\hline Gl 664 & 6.33 & 1.16 & K5 Ve & 6.0 & 288 & 0.255 & 0.032 & 157 & 3 & -0.60 & 0.11 & 130.6 & 5.6 \\
\hline Gl 666 A & 5.53 & 0.77 & G8 V & 8.8 & 251 & 0.029 & 0.013 & 8 & 44 & -0.26 & 0.48 & 20.1 & 1.9 \\
\hline Gl 666 B & 8.69 & 1.41 & M0 V & 8.8 & 251 & 0.029 & 0.013 & 8 & 44 & -0.26 & 0.48 & 20.1 & 1.9 \\
\hline Gl 667 A & 6.29 & 1.04 & $\mathrm{~K} 3 \mathrm{~V}$ & 7.2 & 307 & 0.206 & 0.029 & 120 & 5 & -0.61 & 0.11 & 104.4 & 6.4 \\
\hline Gl 667 B & 7.20 & & K5 V & 7.2 & 307 & 0.206 & 0.029 & 120 & 5 & -0.61 & 0.11 & 104.4 & 6.4 \\
\hline Gl $667 \mathrm{C}$ & 10.24 & 1.57 & M2.5 & 7.2 & 307 & 0.206 & 0.029 & 120 & 17 & -0.61 & 0.11 & 104.4 & 6.4 \\
\hline BPM 78873 & 10.59 & 1.44 & $\mathrm{~K} 7$ & 20.7 & 445 & 0.035 & 0.011 & 13 & 23 & -0.56 & 0.25 & 18.5 & 9.5 \\
\hline LTT 15124 & 13.02 & & $\mathrm{~m}$ & 19.5 & 613 & 0.031 & 0.009 & 19 & 11 & -0.57 & 0.22 & 16.2 & 7.4 \\
\hline Gl 669 A & 11.42 & 1.55 & $\mathrm{dM} 4 \mathrm{e}$ & 10.7 & 691 & 0.594 & 0.031 & 999 & 13 & -0.16 & 0.05 & 443.5 & 60.3 \\
\hline Gl 669 B & 12.97 & 1.64 & dM5 e & 10.7 & 691 & 0.594 & 0.031 & 999 & 7 & -0.16 & 0.05 & 443.5 & 60.3 \\
\hline Gl $670 \mathrm{~A}$ & 4.41 & 0.39 & $\mathrm{~F} 2 \mathrm{~V}$ & 17.4 & 318 & 0.112 & 0.021 & 52 & 18 & -0.60 & 0.16 & 57.7 & 20.9 \\
\hline Gl $670 \mathrm{~B}$ & 8.90 & & K3 & 17.4 & 318 & 0.112 & 0.021 & 52 & 18 & -0.60 & 0.16 & 57.7 & 20.9 \\
\hline Gl 673 & 7.53 & 1.36 & K7 V & 7.7 & 445 & 0.090 & 0.018 & 40 & 3 & -0.37 & 0.18 & 57.5 & 4.1 \\
\hline Gl 674 & 9.37 & 1.53 & M3 & 4.5 & 193 & 0.267 & 0.041 & 76 & 2 & -0.60 & 0.13 & 136.8 & 3.4 \\
\hline LTT 15193 & 13.35 & & $\mathrm{~m}$ & 21.2 & 883 & 0.038 & 0.008 & 30 & 9 & -0.37 & 0.20 & 23.9 & 12.9 \\
\hline Gl 679 & 6.56 & 0.65 & G5 V & 23.7 & 840 & 0.024 & 0.007 & 17 & 17 & -0.80 & 0.15 & 10.0 & 6.7 \\
\hline Gl 681 & 2.08 & 0.15 & A5 III & 14.3 & 542 & 0.124 & 0.016 & 111 & 7 & -0.60 & 0.11 & 63.7 & 15.6 \\
\hline Gl 683.1 & 7.17 & 0.65 & G5 V & 33.6 & 234 & 0.129 & 0.027 & 46 & 22 & -0.39 & 0.18 & 80.3 & 108.2 \\
\hline Gl $684 \mathrm{~A}$ & 5.34 & 0.56 & G0 Va & 14.1 & 2942 & 0.345 & 0.012 & 999 & 10 & -0.49 & 0.03 & 197.2 & 46.8 \\
\hline Gl 684 B & 8.06 & 1.10 & K3 V & 14.1 & 2942 & 0.345 & 0.012 & 999 & 10 & -0.49 & 0.03 & 197.2 & 46.8 \\
\hline Gl 685 & 9.97 & 1.47 & M1 Ve & 14.1 & 2947 & 0.023 & 0.004 & 55 & 8 & -0.58 & 0.11 & 12.0 & 2.9 \\
\hline Gl 686.1A & 10.00 & 1.33 & dM0 e & 22.1 & 638 & 0.025 & 0.008 & 14 & 31 & -0.56 & 0.26 & 13.2 & 7.8 \\
\hline Gl 686.1B & 10.22 & 1.38 & & 22.1 & 638 & 0.025 & 0.008 & 14 & 31 & -0.56 & 0.26 & 13.2 & 7.8 \\
\hline Gl 686.2 & 4.77 & 0.40 & $\mathrm{~F} 2 \mathrm{~V}$ & 21.9 & 164 & 0.128 & 0.033 & 32 & 13 & -0.31 & 0.27 & 85.1 & 48.8 \\
\hline Gl 687 & 9.18 & 1.50 & M3.5 V & 4.5 & 5788 & 0.062 & 0.004 & 374 & 8 & -0.63 & 0.05 & 30.8 & 0.8 \\
\hline Gl 688 & 6.52 & 0.96 & K3 V & 10.7 & 440 & 0.048 & 0.013 & 19 & 11 & -0.71 & 0.18 & 21.9 & 3.0 \\
\hline Gl 689 & 8.62 & 1.10 & $\mathrm{dK} 8$ & 19.0 & 2894 & 0.017 & 0.003 & 23 & 14 & -0.65 & 0.14 & 8.3 & 3.6 \\
\hline HD 160922 & 4.80 & 0.43 & F5 V & 23.5 & 4848 & 0.465 & 0.010 & 999 & 8 & -0.28 & 0.02 & 317.8 & 209.5 \\
\hline $\mathrm{AC}+61: 27026 \mathrm{~A}$ & 10.28 & 1.23 & $\mathrm{dK} 8$ & 24.5 & 2881 & 0.634 & 0.016 & 999 & 7 & -0.15 & 0.02 & 476.7 & 342.6 \\
\hline $\mathrm{AC}+61: 27026 \mathrm{~B}$ & 14.70 & & & 24.5 & 2881 & 0.634 & 0.016 & 999 & 10 & -0.15 & 0.02 & 476.7 & 342.6 \\
\hline Gl 690 A & 9.20 & 1.10 & dM0 & 23.9 & 3280 & 0.011 & 0.003 & 18 & 10 & -0.82 & 0.12 & 4.4 & 3.0 \\
\hline Wo 9599 & 8.37 & 0.93 & K0 & 26.5 & 5029 & 0.036 & 0.003 & 179 & 1 & -0.38 & 0.08 & 22.5 & 18.9 \\
\hline G140-009 & 10.67 & & M2 & 19.7 & 350 & 0.060 & 0.016 & 22 & 5 & -0.70 & 0.20 & 27.8 & 12.9 \\
\hline Gl 694 & 10.47 & 1.53 & dM3.5 & 9.5 & 1109 & 0.024 & 0.006 & 17 & 7 & -0.79 & 0.15 & 9.7 & 1.0 \\
\hline Gl $694.1 \mathrm{~A}$ & 4.58 & 0.42 & F5 IV-V & 22.0 & 3397 & 0.125 & 0.007 & 600 & 9 & -0.50 & 0.04 & 70.8 & 41.1 \\
\hline Gl 694.1B & 5.79 & 0.53 & $\mathrm{~F} 8 \mathrm{~V}$ & 22.3 & 3397 & 0.125 & 0.007 & 600 & 22 & -0.50 & 0.04 & 70.8 & 42.2 \\
\hline Gl 694.2 & 10.72 & 1.49 & $\mathrm{dM} 1.5$ & 22.5 & 1279 & 0.009 & 0.004 & 8 & 28 & 0.06 & 0.37 & 7.9 & 4.8 \\
\hline Gl 695 A & 3.42 & 0.75 & G5 IV & 8.4 & 718 & 0.197 & 0.019 & 232 & 6 & -0.86 & 0.06 & 73.9 & 6.2 \\
\hline Gl 695 B & 10.35 & 1.49 & M3 & 8.4 & 718 & 0.197 & 0.019 & 232 & 27 & -0.86 & 0.06 & 73.9 & 6.2 \\
\hline Gl $695 \mathrm{C}$ & 10.80 & & M4 & 8.4 & 718 & 0.197 & 0.019 & 232 & 27 & -0.86 & 0.06 & 73.9 & 6.2 \\
\hline Gl 697 & 8.48 & 0.95 & dK5 & 24.9 & 619 & 0.038 & 0.009 & 29 & 9 & -0.34 & 0.21 & 24.7 & 18.3 \\
\hline HD 162521 & 6.36 & 0.45 & $\mathrm{~F} 8 \mathrm{~V}$ & 34.6 & 151 & 0.087 & 0.030 & 16 & 9 & -0.49 & 0.34 & 49.6 & 71.0 \\
\hline Gl 698 & 9.22 & 1.18 & $\mathrm{dK} 8$ & 23.0 & 271 & 0.030 & 0.013 & 9 & 26 & -0.49 & 0.40 & 17.4 & 11.0 \\
\hline HD 163621 & 7.84 & & G5 & 30.9 & 880 & 1.875 & 0.047 & 999 & 5 & -0.05 & 0.03 & 1508.4 & 1719.2 \\
\hline HD 163840 & 6.30 & 0.64 & G2 V & 28.6 & 651 & 0.020 & 0.007 & 14 & 35 & -1.00 & 0.37 & 6.1 & 5.9 \\
\hline Gl 699 & 9.55 & 1.74 & M5 V & 1.8 & 399 & 0.030 & 0.011 & 13 & 11 & -0.75 & 0.30 & 12.9 & 0.1 \\
\hline G204-039 & 11.79 & 1.56 & $\mathrm{~m}$ & 13.6 & 1065 & 0.025 & 0.007 & 19 & 15 & -0.58 & 0.19 & 13.2 & 2.9 \\
\hline Gl 699.2 & 4.62 & 0.38 & F3 V & 23.2 & 343 & 0.197 & 0.025 & 133 & 7 & -0.04 & 0.13 & 159.3 & 102.6 \\
\hline Gl $700.1 \mathrm{~A}$ & 5.24 & 0.38 & dF3 & 52.1 & 276 & 0.076 & 0.019 & 32 & 4 & -0.14 & 0.24 & 57.5 & 186.7 \\
\hline Gl 700.1B & 5.93 & & & 52.1 & 276 & 0.076 & 0.019 & 32 & 4 & -0.14 & 0.24 & 57.5 & 186.7 \\
\hline Gl 701 & 9.38 & 1.52 & $\mathrm{dM} 2$ & 7.8 & 310 & 0.031 & 0.012 & 10 & 17 & -0.11 & 0.39 & 23.7 & 1.7 \\
\hline Gl 702 A & 4.21 & 0.86 & K0 Ve & 5.1 & 347 & 1.649 & 0.127 & 410 & 7 & -0.52 & 0.07 & 915.9 & 28.3 \\
\hline Gl $702 \mathrm{~B}$ & 6.00 & 1.15 & $\mathrm{~K} 5 \mathrm{Ve}$ & 5.1 & 347 & 1.649 & 0.127 & 410 & 7 & -0.52 & 0.07 & 915.9 & 28.3 \\
\hline Gl 702.1 & 5.95 & 0.62 & G5 V & 17.4 & 124 & 0.585 & 0.075 & 131 & 12 & -0.27 & 0.13 & 402.7 & 145.2 \\
\hline
\end{tabular}




\begin{tabular}{|c|c|c|c|c|c|c|c|c|c|c|c|c|c|}
\hline Name & $V$ & $B-V$ & Sp. type & $d(\mathrm{pc})$ & Exp. & $C R$ & $\pm C R$ & $\mathrm{Li}$ & $\Delta$ & $h r$ & $\Delta h r$ & $f_{\mathrm{x}-14}$ & $L_{\mathrm{x} 27}$ \\
\hline Gl 702.2 & 6.80 & 0.62 & $\mathrm{G} 2 \mathrm{~V}$ & 24.4 & 348 & 0.039 & 0.015 & 12 & 11 & -0.38 & 0.36 & 24.6 & 17.5 \\
\hline GJ 1224 & 13.63 & 1.79 & $\mathrm{~m}$ & 7.5 & 296 & 0.211 & 0.029 & 104 & 5 & -0.45 & 0.12 & 124.8 & 8.5 \\
\hline Wo 9615 A & 3.73 & 0.12 & $\mathrm{~A} 4 \mathrm{~V}$ & 25.4 & 401 & 0.021 & 0.010 & 7 & 31 & -0.58 & 0.30 & 11.2 & 8.6 \\
\hline Wo $9615 \mathrm{~B}$ & 14.00 & & & 25.4 & 401 & 0.021 & 0.010 & 7 & 31 & -0.58 & 0.30 & 11.2 & 8.6 \\
\hline Gl $704 \mathrm{~A}$ & 5.09 & 0.50 & F7 V & 15.6 & 719 & 0.016 & 0.006 & 9 & 17 & -1.00 & 0.57 & 4.9 & 1.4 \\
\hline Gl $704 \mathrm{~B}$ & 8.45 & 1.10 & $\mathrm{~K} 5 \mathrm{~V}$ & 15.6 & 719 & 0.016 & 0.006 & 9 & 17 & -1.00 & 0.57 & 4.9 & 1.4 \\
\hline LP $390-16$ & 13.32 & & $\mathrm{~m}$ & 13.9 & 554 & 0.300 & 0.025 & 308 & 11 & 0.00 & 0.08 & 249.1 & 57.2 \\
\hline Gl 708.1 & 5.03 & 0.38 & F5 V & 23.5 & 5748 & 0.242 & 0.007 & 999 & 6 & -0.18 & 0.03 & 178.2 & 117.5 \\
\hline Gl 710 & 9.66 & 1.37 & dM1 & 19.3 & 308 & 0.041 & 0.014 & 13 & 17 & -0.16 & 0.39 & 30.2 & 13.5 \\
\hline G258-033 & 13.48 & 1.83 & $\mathrm{~m}$ & 7.3 & 9325 & 0.056 & 0.003 & 494 & 2 & -0.50 & 0.04 & 31.8 & 2.0 \\
\hline G021-013 & 11.84 & 1.56 & $\mathrm{~g}$ & & 321 & 0.019 & 0.009 & 8 & 31 & -1.00 & 1.28 & 5.6 & \\
\hline Gl $713 \mathrm{AB}$ & 3.57 & 0.49 & F7 V & 8.1 & 2629 & 0.156 & 0.009 & 506 & 20 & -0.76 & 0.04 & 66.7 & 5.2 \\
\hline Gl 716 & 6.81 & 0.85 & $\mathrm{~K} 3 \mathrm{~V}$ & 13.2 & 296 & 0.050 & 0.016 & 15 & 23 & -0.22 & 0.37 & 35.6 & 7.4 \\
\hline G205-028 & 11.99 & & $\mathrm{~m}$ & 12.5 & 727 & 0.011 & 0.005 & 8 & 51 & -0.73 & 0.33 & 4.8 & 0.9 \\
\hline Gl 719 & 8.10 & 1.22 & $\mathrm{~K} 6 \mathrm{Ve}$ & 16.4 & 1085 & 2.414 & 0.048 & 999 & 2 & -0.01 & 0.02 & 1993.2 & 643.0 \\
\hline LP $229-17$ & 11.42 & 1.42 & M3 p & 7.7 & 703 & 0.019 & 0.006 & 17 & 24 & -0.52 & 0.26 & 10.4 & 0.7 \\
\hline Gl $720 \mathrm{~A}$ & 9.85 & 1.42 & $\mathrm{dM} 2$ & 15.5 & 1183 & 0.014 & 0.005 & 14 & 7 & -0.70 & 0.21 & 6.3 & 1.8 \\
\hline G141-021 & 12.46 & & M3.5 & 10.4 & 335 & 0.111 & 0.021 & 52 & 10 & -0.43 & 0.19 & 66.8 & 8.6 \\
\hline Gl 722 & 5.86 & 0.68 & G5 V V & 13.0 & 283 & 0.045 & 0.016 & 15 & 16 & -0.67 & 0.21 & 21.4 & 4.3 \\
\hline Gl 722.1 & 8.34 & 0.82 & dK0 e & 28.5 & 1019 & 0.011 & 0.004 & 9 & 24 & -0.76 & 0.30 & 4.6 & 4.5 \\
\hline GJ $1230 \mathrm{~A}$ & 12.40 & 1.71 & $\mathrm{k}-\mathrm{m}$ & 8.3 & 454 & 0.179 & 0.022 & 156 & 13 & -0.16 & 0.12 & 133.5 & 10.9 \\
\hline GJ $1230 \mathrm{~B}$ & 14.00 & & $\mathrm{~m}$ & 8.3 & 454 & 0.179 & 0.022 & 156 & 16 & -0.16 & 0.12 & 133.5 & 10.9 \\
\hline LP 25-02 & 13.22 & 1.72 & $\mathrm{M} 4 \mathrm{e}$ & 14.1 & 1234 & 0.113 & 0.011 & 191 & 15 & -0.38 & 0.09 & 71.3 & 16.9 \\
\hline G205-035 & 13.42 & & $\mathrm{~m}$ & 14.9 & 900 & 0.051 & 0.009 & 57 & 19 & -0.34 & 0.16 & 33.0 & 8.8 \\
\hline G141-029 & 12.81 & & $\mathrm{~m}$ & 10.7 & 451 & 0.131 & 0.018 & 110 & 11 & -0.16 & 0.14 & 98.1 & 13.5 \\
\hline Gl $725 \mathrm{~A}$ & 8.90 & 1.52 & $\mathrm{dM} 4$ & 3.6 & 1781 & 0.049 & 0.006 & 91 & 23 & -0.80 & 0.07 & 19.9 & 0.3 \\
\hline Gl $725 \mathrm{~B}$ & 9.71 & 1.59 & dM5 & 3.5 & 1781 & 0.049 & 0.006 & 91 & 9 & -0.80 & 0.07 & 19.9 & 0.3 \\
\hline Gl 725.2 & 4.19 & 0.46 & $\mathrm{~F} 6 \mathrm{~V}$ & 19.1 & 272 & 0.376 & 0.039 & 294 & 6 & -0.16 & 0.11 & 280.9 & 122.4 \\
\hline Gl 726 & 8.81 & 1.29 & $\mathrm{~K} 7$ & 14.1 & 346 & 0.033 & 0.011 & 12 & 43 & -1.00 & 0.66 & 9.9 & 2.4 \\
\hline Gl 727 & 7.97 & 1.07 & $\mathrm{dK} 4$ & 17.3 & 280 & 0.076 & 0.020 & 22 & 65 & -0.11 & 0.24 & 58.4 & 20.9 \\
\hline Gl 729 & 10.46 & 1.72 & $\mathrm{dM} 4.5 \mathrm{e}$ & 3.0 & 326 & 0.942 & 0.081 & 334 & 14 & -0.43 & 0.08 & 568.1 & 6.0 \\
\hline Gl 732.1 & 5.50 & 0.84 & G9 IVa & 26.1 & 785 & 0.415 & 0.024 & 775 & 6 & -0.20 & 0.06 & 300.7 & 245.2 \\
\hline Gl 735 & 10.11 & 1.53 & dM3 e & 11.6 & 476 & 1.392 & 0.055 & 999 & 1 & -0.20 & 0.04 & 1009.2 & 162.1 \\
\hline Gl $737 \mathrm{~A}$ & 9.45 & 1.42 & $\mathrm{~K} 7 \mathrm{~V}$ & 12.6 & 115 & 0.092 & 0.032 & 16 & 74 & -1.00 & 0.89 & 27.8 & 5.3 \\
\hline Gl $737 \mathrm{~B}$ & 10.00 & & K5 V & 12.6 & 115 & 0.092 & 0.032 & 16 & 74 & -1.00 & 0.89 & 27.8 & 5.3 \\
\hline Wo 9638 & 8.09 & 0.91 & $\mathrm{~K} 2$ & 21.5 & 648 & 1.878 & 0.052 & 999 & 75 & -0.11 & 0.03 & 1451.1 & 799.5 \\
\hline Gl $738 \mathrm{~A}$ & 5.34 & 0.59 & F9 V & 15.0 & 729 & 0.101 & 0.014 & 97 & 6 & -0.77 & 0.09 & $\begin{array}{r}1401.1 \\
42.8\end{array}$ & 11.5 \\
\hline Gl $738 \mathrm{~B}$ & 7.70 & & $\mathrm{~K} 1 \mathrm{~V}$ & 15.0 & 729 & 0.101 & 0.014 & 97 & 6 & -0.77 & 0.09 & 42.8 & 11.5 \\
\hline Gl 740 & 9.22 & 1.46 & $\mathrm{M} 2 \mathrm{~V}$ & 11.1 & 442 & 0.029 & 0.010 & 13 & 35 & -0.08 & 0.32 & 23.2 & 3.4 \\
\hline Steph 1676 & 10.60 & 1.42 & M0 & 25.3 & 2589 & 0.165 & 0.009 & 765 & 6 & -0.11 & 0.05 & 127.8 & 98.0 \\
\hline $\mathrm{Gl} 743.1 \mathrm{~A}$ & 4.87 & 0.52 & F8 V & 17.9 & 259 & 0.044 & 0.015 & 16 & 12 & -0.74 & 0.28 & 19.3 & 7.4 \\
\hline Gl $743.1 \mathrm{~B}$ & 5.00 & & $\mathrm{~F} 8 \mathrm{~V}$ & 17.9 & 259 & 0.044 & 0.015 & 16 & 12 & -0.74 & 0.28 & 19.3 & 7.4 \\
\hline HD 177724 & 2.99 & 0.01 & $\mathrm{~A} 0 \mathrm{Vn}$ & 25.5 & 435 & 0.141 & 0.020 & 91 & 41 & -0.11 & 0.14 & 109.0 & 84.8 \\
\hline Gl 744 & 6.15 & 0.71 & G5 IV & 17.2 & 260 & 0.024 & 0.012 & 7 & 32 & & & 19.8 & 7.0 \\
\hline HD 177996 & 7.88 & 0.86 & $\mathrm{~K} 1 \mathrm{~V}$ & 31.7 & 215 & 0.421 & 0.046 & 197 & 5 & -0.06 & 0.11 & 336.7 & 406.0 \\
\hline HD 178076 & 7.82 & & Ko V & 28.8 & 184 & 0.120 & 0.030 & 28 & 23 & -0.36 & 0.23 & 77.1 & 76.7 \\
\hline Gl 746 & 6.07 & 0.70 & G5 V & 21.0 & 450 & 0.047 & 0.012 & 31 & 24 & -0.29 & 0.23 & 32.0 & 16.8 \\
\hline Gl $747 \mathrm{~A}$ & 11.86 & 1.70 & M3.5 & 8.2 & 701 & 0.025 & 0.007 & 19 & 2 & -1.00 & 0.57 & 7.5 & 0.6 \\
\hline Gl $747 \mathrm{~B}$ & 12.16 & & M5 & 8.2 & 701 & 0.025 & 0.007 & 19 & 2 & -1.00 & 0.57 & 7.5 & 0.6 \\
\hline $\mathrm{BD}+79615$ & 9.72 & 1.10 & $\mathrm{dK} 8$ & 32.5 & 1164 & 0.014 & 0.005 & 15 & 9 & -0.01 & 0.36 & 11.8 & 14.9 \\
\hline Gl 748.1 & 5.13 & 0.31 & $\mathrm{~F} 2 \mathrm{~V}$ & 27.3 & 1584 & 0.127 & 0.010 & 351 & 7 & -0.12 & 0.08 & 97.5 & 87.1 \\
\hline GJ 1233 & 7.04 & 0.79 & G8 V & 20.0 & 1423 & 0.155 & 0.011 & 360 & 10 & -0.40 & 0.07 & 96.3 & 46.1 \\
\hline Wo $9652 \mathrm{~A}$ & 11.55 & & M3 & 19.0 & 517 & 0.550 & 0.048 & 349 & 6 & -0.19 & 0.09 & 401.5 & 174.3 \\
\hline Wo $9652 \mathrm{~B}$ & 13.27 & & M3.5 & 19.0 & 517 & 0.550 & 0.048 & 349 & 46 & -0.19 & 0.09 & 401.5 & 174.3 \\
\hline Gl 754.2 & 6.31 & 0.68 & G8 V & 25.3 & 515 & 0.105 & 0.017 & 105 & 24 & -0.26 & 0.15 & 72.5 & 55.3 \\
\hline Gl 755 & 6.48 & 0.62 & G5 V & 20.8 & 291 & 0.627 & 0.049 & 421 & 12 & -0.09 & 0.08 & 490.8 & 254.9 \\
\hline Gl 759 & 5.16 & 0.77 & G8 IV & 15.2 & 488 & 0.029 & 0.009 & 13 & 37 & -0.62 & 0.34 & 14.4 & 3.9 \\
\hline Gl 760 & 3.36 & 0.32 & F0 IV & 15.4 & 382 & 0.241 & 0.027 & 185 & 11 & -0.56 & 0.09 & 129.0 & 36.4 \\
\hline LTT 15678 & 13.09 & & $\mathrm{~m}$ & 17.9 & 193 & 0.054 & 0.021 & 12 & 46 & -0.90 & 0.46 & 19.0 & 7.3 \\
\hline Gl 764 & 4.68 & 0.79 & $\mathrm{~K} 0 \mathrm{~V}$ & 5.8 & 1448 & 0.256 & 0.014 & 694 & 2 & -0.80 & 0.03 & 104.1 & 4.1 \\
\hline Gl 765 A & 4.48 & 0.38 & $\mathrm{~F} 4 \mathrm{~V}$ & 18.6 & 853 & 0.323 & 0.021 & 562 & 8 & -0.43 & 0.06 & 195.1 & 80.6 \\
\hline Gl $765 \mathrm{~B}$ & 13.00 & & & 18.6 & 853 & 0.323 & 0.021 & 562 & 8 & -0.43 & 0.06 & 195.1 & 80.6 \\
\hline HD 185501 & 7.47 & & G5 & 32.3 & 415 & 0.032 & 0.011 & 15 & 23 & -0.18 & 0.32 & 23.4 & 29.1 \\
\hline CD-45 13383 & 10.01 & 1.34 & M1 & 28.8 & 321 & 0.025 & 0.011 & 10 & 14 & -0.86 & 0.51 & 9.5 & 9.5 \\
\hline Gl 765.2 & 8.08 & 0.88 & $\mathrm{dK} 0$ & 30.0 & 1583 & 0.020 & 0.004 & 22 & 13 & -0.84 & 0.15 & 7.8 & 8.4 \\
\hline Wo 9666 & 5.49 & 0.46 & F5 IV & 36.6 & 305 & 0.100 & 0.021 & 47 & 19 & -0.44 & 0.17 & 59.6 & 95.7 \\
\hline Gl $765.4 \mathrm{~A}$ & 8.35 & 0.99 & $\mathrm{~K} 3 \mathrm{~V}$ & 20.4 & 479 & 0.045 & 0.012 & 24 & 27 & -0.85 & 0.20 & 17.2 & 8.5 \\
\hline Gl $765.4 \mathrm{~B}$ & 8.54 & & $\mathrm{~K} 3 \mathrm{~V}$ & 20.4 & 479 & 0.045 & 0.012 & 24 & 27 & -0.85 & 0.20 & 17.2 & 8.5 \\
\hline Gl $767.1 \mathrm{~A}$ & 4.99 & 0.47 & F5 IV-V & 20.9 & 468 & 0.136 & 0.018 & 109 & 6 & -0.29 & 0.14 & 91.8 & 47.9 \\
\hline Gl $767.1 \mathrm{~B}$ & 8.56 & 1.04 & $\mathrm{dK} 6$ & 20.9 & 468 & 0.136 & 0.018 & 109 & 33 & -0.29 & 0.14 & 91.8 & 47.9 \\
\hline G125-036 & 12.88 & & $\mathrm{~m}$ & 24.4 & 493 & 0.138 & 0.018 & 128 & 7 & -0.21 & 0.13 & 99.1 & 70.9 \\
\hline Gl 768 & 0.77 & 0.22 & A7 IV-V & 5.1 & 486 & 0.148 & 0.020 & 79 & 4 & -1.00 & 0.06 & 44.5 & 1.4 \\
\hline Gl $768.1 \mathrm{~A}$ & 5.11 & 0.55 & F8 V & 19.4 & 401 & 0.046 & 0.013 & 16 & 22 & -0.56 & 0.25 & 24.8 & 11.1 \\
\hline Gl $768.1 B$ & 13.10 & & M3 & 19.4 & 401 & 0.046 & 0.013 & 16 & 22 & -0.56 & 0.25 & 24.8 & 11.1 \\
\hline GJ 1243 & 12.83 & 1.64 & $\mathrm{~m}$ & 11.9 & 847 & 0.098 & 0.012 & 129 & 3 & -0.26 & 0.11 & 67.9 & 11.5 \\
\hline Gl 770 & 6.17 & 1.02 & $\mathrm{~K} 3 / 4 \mathrm{~V}$ & 14.2 & 276 & 0.416 & 0.041 & 272 & 6 & -0.48 & 0.09 & 239.8 & 58.1 \\
\hline GJ $1245 \mathrm{~A}$ & 13.41 & 1.90 & $\mathrm{M} 5.5 \mathrm{~V} \mathrm{e}$ & 4.5 & 797 & 0.198 & 0.017 & 331 & 11 & -0.37 & 0.08 & 125.8 & 3.1 \\
\hline GJ $1245 \mathrm{~B}$ & 14.01 & 1.98 & $\mathrm{~m}$ & 4.5 & 797 & 0.198 & 0.017 & 331 & 7 & -0.37 & 0.08 & 125.8 & 3.1 \\
\hline Gl $771 \mathrm{~A}$ & 3.72 & 0.86 & G8 IV & 13.7 & 343 & 0.248 & 0.029 & 202 & 15 & -0.34 & 0.11 & 161.3 & 36.3 \\
\hline Gl 771 B & 11.40 & & M3 & 13.7 & 343 & 0.248 & 0.029 & 202 & 15 & -0.34 & 0.11 & 161.3 & 36.3 \\
\hline
\end{tabular}




\begin{tabular}{|c|c|c|c|c|c|c|c|c|c|c|c|c|c|}
\hline Name & $V$ & $B-V$ & Sp. type & $d(\mathrm{pc})$ & Exp. & $C R$ & $\pm C R$ & $\mathrm{Li}$ & $\Delta$ & $h r$ & $\Delta h r$ & $f_{\mathrm{x}-14}$ & $L_{\mathrm{x} 27}$ \\
\hline Gl 773.2 & 7.90 & 0.80 & $\mathrm{~K} 0 \mathrm{Ve}$ & 25.5 & 528 & 0.241 & 0.023 & 262 & 8 & -0.22 & 0.10 & 172.0 & 133.9 \\
\hline Gl 773.4 & 5.66 & 0.49 & $\mathrm{~F} 8 \mathrm{~V}$ & 20.9 & 250 & 1.789 & 0.125 & 622 & 3 & 0.05 & 0.07 & 1534.1 & 799.9 \\
\hline HD 189733 & 7.67 & & G5 & 19.3 & 412 & 0.108 & 0.018 & 58 & 7 & -0.48 & 0.15 & 62.4 & 27.7 \\
\hline Gl 775 & 7.46 & 1.14 & $\mathrm{~K} 4 \mathrm{~V}$ & 13.1 & 317 & 0.072 & 0.018 & 35 & 4 & -0.74 & 0.17 & 31.8 & 6.5 \\
\hline Gl 779 & 5.80 & 0.61 & G1 V & 17.7 & 374 & 0.056 & 0.015 & 23 & 30 & -1.00 & 0.15 & 16.9 & 6.3 \\
\hline GJ 1249 & 6.17 & 0.65 & G5 IV & 18.9 & 710 & 0.527 & 0.028 & 970 & 6 & -0.09 & 0.06 & 412.6 & 175.8 \\
\hline Gl 780 & 3.56 & 0.76 & $\mathrm{G} 8 \mathrm{~V}$ & 6.1 & 124 & 0.073 & 0.028 & 10 & 58 & -0.52 & 0.41 & 40.5 & 1.8 \\
\hline Gl 781 & 11.99 & 1.54 & dM3 e & 16.5 & 1209 & 0.107 & 0.011 & 223 & 5 & -0.42 & 0.09 & 65.4 & 21.2 \\
\hline Gl $781.1 \mathrm{~A}$ & 12.25 & 1.55 & M3 & 14.6 & 198 & 0.204 & 0.035 & 60 & 45 & -0.16 & 0.17 & 152.6 & 38.9 \\
\hline Gl 781.1B & 12.50 & 1.63 & M3.5 & 14.6 & 198 & 0.204 & 0.035 & 60 & 6 & -0.16 & 0.17 & 152.6 & 38.9 \\
\hline GJ 1250 & 14.88 & 1.82 & $\mathrm{~m}$ & 21.6 & 604 & 0.021 & 0.008 & 11 & 20 & -0.35 & 0.33 & 13.7 & 7.7 \\
\hline HD 192020 & 7.95 & 0.86 & G8 V & 24.8 & 739 & 0.065 & 0.012 & 51 & 5 & -0.25 & 0.16 & 45.3 & 33.2 \\
\hline Gl 784 & 7.97 & 1.43 & M0 V & 6.2 & 321 & 0.093 & 0.021 & 28 & 16 & -0.67 & 0.19 & 44.1 & 2.0 \\
\hline Gl 785 & 5.73 & 0.88 & $\mathrm{~K} 0 \mathrm{~V}$ & 8.8 & 126 & 0.062 & 0.027 & 10 & 61 & -1.00 & 0.84 & 18.6 & 1.7 \\
\hline Gl 786 & 8.88 & 1.33 & dM0 & 16.8 & 1432 & 0.010 & 0.003 & 14 & 17 & -0.52 & 0.23 & 5.8 & 2.0 \\
\hline LTT 15944 & 11.79 & & $\mathrm{~m}$ & 16.6 & 503 & 0.017 & 0.008 & 12 & 51 & -0.58 & 0.24 & 9.0 & 3.0 \\
\hline $\mathrm{Gl} 791.1 \mathrm{~A}$ & 4.78 & 0.38 & F2 IV & 30.3 & 412 & 0.098 & 0.018 & 49 & 8 & -0.56 & 0.15 & 52.1 & 57.3 \\
\hline Gl 791.1B & 10.00 & & & 30.3 & 412 & 0.098 & 0.018 & 49 & 8 & -0.56 & 0.15 & 52.1 & 57.3 \\
\hline Gl 791.2 & 13.05 & 1.65 & dM6 e & 8.8 & 401 & 0.141 & 0.021 & 85 & 5 & -0.44 & 0.13 & 84.4 & 7.8 \\
\hline Wo 9697 A & 10.28 & 1.34 & dM1 & 23.1 & 597 & 0.019 & 0.007 & 12 & 17 & -0.45 & 0.41 & 11.4 & 7.3 \\
\hline Wo 9697 B & 10.70 & & & 23.1 & 597 & 0.019 & 0.007 & 12 & 17 & -0.45 & 0.41 & 11.4 & 7.3 \\
\hline Gl 791.3 & 9.23 & 1.13 & dK 8 & 26.2 & 628 & 0.016 & 0.007 & 8 & 10 & -0.56 & 0.31 & 8.4 & 6.9 \\
\hline Gl 793 & 10.56 & 1.56 & dM3 & 8.0 & 1175 & 0.130 & 0.012 & 259 & 4 & -0.45 & 0.08 & 76.9 & 5.8 \\
\hline Gl 793.1 & 7.09 & 0.79 & G9 V & 22.2 & 790 & 0.013 & 0.005 & 13 & 22 & -1.00 & 9.99 & 4.1 & 2.4 \\
\hline Gl 794 & 11.52 & -0.07 & DA3 & 14.8 & 572 & 0.010 & 0.006 & 7 & 11 & -1.00 & 2.60 & 3.0 & 0.8 \\
\hline Gl $795 \mathrm{~A}$ & 8.18 & 1.22 & $\mathrm{~K} 4 \mathrm{~V}$ & 18.6 & 415 & 0.056 & 0.013 & 32 & 13 & -0.33 & 0.21 & 36.8 & 15.2 \\
\hline Gl 795 B & 9.40 & & $\mathrm{~K} 8 \mathrm{~V}$ & 18.6 & 415 & 0.056 & 0.013 & 32 & 13 & -0.33 & 0.21 & 36.8 & 15.2 \\
\hline GJ $1255 \mathrm{AB}$ & 8.00 & 0.86 & $\mathrm{~K} 0 \mathrm{~V}$ & 27.6 & 988 & 1.416 & 0.039 & 999 & 7 & -0.05 & 0.03 & 1139.2 & 1040.1 \\
\hline GJ $1255 \mathrm{C}$ & 10.40 & & & 27.6 & 988 & 1.416 & 0.039 & 999 & 7 & -0.05 & 0.03 & 1139.2 & 1040.1 \\
\hline Gl 799 A & 10.99 & 1.57 & $\mathrm{dM} 4.5 \mathrm{e}$ & 10.2 & 288 & 3.908 & 0.120 & 999 & 1 & -0.19 & 0.03 & 2854.0 & 357.0 \\
\hline Gl 799 B & 11.00 & & $\mathrm{dM} 4.5 \mathrm{e}$ & 10.2 & 288 & 3.908 & 0.120 & 999 & 1 & -0.19 & 0.03 & 2854.0 & 357.0 \\
\hline GJ 1257 & 9.70 & 1.11 & K5 V & 23.0 & 169 & 0.525 & 0.057 & 224 & 16 & -0.29 & 0.11 & 355.5 & 225.8 \\
\hline $\mathrm{BD}+562471$ & 10.29 & 1.36 & K7 & 24.0 & 941 & 0.114 & 0.012 & 178 & 20 & -0.12 & 0.10 & 87.2 & 60.0 \\
\hline HD 197214 & 6.95 & 0.67 & G5 V & 22.4 & 427 & 0.027 & 0.011 & 9 & 21 & -0.74 & 0.32 & 11.9 & 7.2 \\
\hline Gl 802 & 14.68 & 1.79 & dM5 e & 15.9 & 956 & 0.045 & 0.008 & 48 & 25 & -0.42 & 0.15 & 27.7 & 8.3 \\
\hline Gl 803 & 8.81 & 1.42 & Mo Ve & 9.9 & 414 & 5.952 & 0.121 & 999 & 1 & -0.08 & 0.02 & 4693.7 & 554.9 \\
\hline Gl 805 & 4.13 & 0.43 & F5 V & 14.7 & 224 & 0.634 & 0.056 & 408 & 11 & -0.14 & 0.09 & 479.9 & 123.4 \\
\hline Gl 807 & 3.43 & 0.92 & K0 IVe & 14.3 & 841 & 0.011 & 0.005 & 10 & 29 & -0.78 & 0.23 & 4.7 & 1.2 \\
\hline G231-017 & 9.74 & 1.32 & K5 & 22.4 & 862 & 0.010 & 0.004 & 8 & 89 & -1.00 & 0.44 & 3.0 & 1.8 \\
\hline HD 198809 & 4.58 & 0.83 & G7 III CN & 66.2 & 515 & 0.225 & 0.023 & 275 & 14 & 0.20 & 0.10 & 210.9 & 1106.8 \\
\hline Gl 809 & 8.55 & 1.48 & $\mathrm{M} 2 \mathrm{Ve}$ & 7.0 & 1157 & 0.084 & 0.009 & 139 & 7 & -0.54 & 0.09 & 45.7 & 2.7 \\
\hline Gl 811 & 5.70 & 0.50 & F8 V & 21.0 & 375 & 0.389 & 0.034 & 352 & 5 & -0.19 & 0.09 & 283.7 & 149.8 \\
\hline Gl $812 \mathrm{~A}$ & 11.91 & 1.51 & M3 & 17.7 & 444 & 0.310 & 0.027 & 303 & 15 & -0.25 & 0.09 & 216.8 & 81.0 \\
\hline Gl 812 B & 16.60 & 1.16 & DC9+ & 17.7 & 444 & 0.310 & 0.027 & 303 & 32 & -0.25 & 0.09 & 216.8 & 81.0 \\
\hline $\mathrm{Gl} 815 \mathrm{~A}$ & 10.34 & 1.52 & dM3 e & 15.1 & 640 & 1.013 & 0.041 & 999 & 1 & -0.24 & 0.04 & 712.9 & 194.6 \\
\hline Gl $815 \mathrm{~B}$ & 11.90 & & & 15.1 & 640 & 1.013 & 0.041 & 999 & 1 & -0.24 & 0.04 & 712.9 & 194.6 \\
\hline Wo 9714 & 9.40 & 1.24 & K5 V & 20.4 & 438 & 0.041 & 0.011 & 26 & 23 & -0.13 & 0.26 & 31.6 & 15.8 \\
\hline G187-013 & 12.29 & & $\mathrm{~m}$ & 16.2 & 616 & 0.050 & 0.011 & 32 & 15 & -0.65 & 0.17 & 24.1 & 7.6 \\
\hline G187-014 & 13.12 & & $\mathrm{~m}$ & 16.2 & 616 & 0.050 & 0.011 & 32 & 56 & -0.65 & 0.17 & 24.1 & 7.6 \\
\hline Gl 816 & 11.23 & 1.49 & M2.5 & 13.8 & 444 & 0.020 & 0.008 & 9 & 26 & -0.78 & 0.33 & 8.2 & 1.9 \\
\hline Gl 816.1A & 7.68 & 0.97 & $\mathrm{~K} 2.5 \mathrm{~V}$ & 19.3 & 481 & 0.171 & 0.021 & 130 & 14 & -0.44 & 0.11 & 102.0 & 45.7 \\
\hline Gl $816.1 B$ & 13.00 & & & 19.3 & 481 & 0.171 & 0.021 & 130 & 14 & -0.44 & 0.11 & 102.0 & 45.7 \\
\hline Gl 816.2A & 5.10 & 0.17 & A5 V & 48.5 & 215 & 0.049 & 0.019 & 10 & 13 & -1.00 & 0.35 & 14.8 & 41.8 \\
\hline Gl 816.2B & 6.50 & & & 48.5 & 215 & 0.049 & 0.019 & 10 & 13 & -1.00 & 0.35 & 14.8 & 41.8 \\
\hline Gl $818.1 \mathrm{~A}$ & 6.40 & 0.59 & G3 IV & 18.7 & 139 & 0.243 & 0.046 & 60 & 17 & -0.38 & 0.16 & 153.1 & 64.2 \\
\hline Gl 818.1B & 6.40 & & & 18.7 & 139 & 0.243 & 0.046 & 60 & 17 & -0.38 & 0.16 & 153.1 & 64.2 \\
\hline Gl $818.1 \mathrm{C}$ & 13.50 & & & 18.7 & 139 & 0.243 & 0.046 & 60 & 17 & -0.38 & 0.16 & 153.1 & 64.2 \\
\hline Gl 819 A & 7.15 & 0.90 & $\mathrm{~K} 1 \mathrm{Ve}$ & 17.6 & 427 & 0.210 & 0.025 & 130 & 16 & -0.42 & 0.11 & 127.7 & 47.5 \\
\hline Gl 819 B & 10.20 & & M0 & 17.6 & 427 & 0.210 & 0.025 & 130 & 16 & -0.42 & 0.11 & 127.7 & 47.5 \\
\hline $\mathrm{Gl} 820 \mathrm{~A}$ & 5.21 & 1.18 & $\mathrm{~K} 5 \mathrm{Ve}$ & 3.5 & 543 & 0.422 & 0.030 & 452 & 10 & -0.72 & 0.05 & 189.5 & 2.8 \\
\hline Gl $820 \mathrm{~B}$ & 6.03 & 1.37 & $\mathrm{~K} 7 \mathrm{Ve}$ & 3.5 & 543 & 0.422 & 0.030 & 452 & 10 & -0.72 & 0.05 & 189.5 & 2.8 \\
\hline Wo $9721 \mathrm{~A}$ & 9.44 & 1.13 & dM2 & 26.4 & 445 & 0.603 & 0.038 & 681 & 10 & -0.06 & 0.07 & 482.1 & 401.5 \\
\hline Wo 9721 B & 13.40 & 1.63 & & 26.4 & 445 & 0.603 & 0.038 & 681 & 10 & -0.06 & 0.07 & 482.1 & 401.5 \\
\hline Wo 9726 & 5.83 & 0.45 & F7 V & 32.4 & 464 & 0.119 & 0.018 & 97 & 12 & -0.51 & 0.12 & 66.7 & 83.6 \\
\hline Gl $822.1 \mathrm{~A}$ & 3.82 & 0.38 & Fo IV & 20.9 & 492 & 0.240 & 0.025 & 210 & 10 & -0.45 & 0.09 & 142.0 & 74.3 \\
\hline Gl 822.1B & 6.42 & 0.60 & G0 $\mathrm{V}$ & 20.9 & 492 & 0.240 & 0.025 & 210 & 10 & -0.45 & 0.09 & 142.0 & 74.3 \\
\hline Gl $822.1 \mathrm{C}$ & 12.00 & 1.53 & M3 & 20.9 & 492 & 0.240 & 0.025 & 210 & 82 & -0.45 & 0.09 & 142.0 & 74.3 \\
\hline LTT 16240 & 12.68 & & $\mathrm{~m}$ & 14.4 & 556 & 0.359 & 0.027 & 506 & 3 & -0.13 & 0.07 & 273.7 & 68.0 \\
\hline LP $341-14$ & 13.49 & & $\mathrm{~m}$ & 14.4 & 556 & 0.359 & 0.027 & 506 & 29 & -0.13 & 0.07 & 273.7 & 68.0 \\
\hline Gl 824 & 7.95 & 1.02 & $\mathrm{dK} 8$ & 16.2 & 353 & 0.031 & 0.012 & 11 & 37 & -0.46 & 0.29 & 18.3 & 5.7 \\
\hline Gl 825 & 6.67 & 1.41 & Mo Ve & 3.9 & 403 & 0.137 & 0.022 & 83 & 3 & -0.73 & 0.10 & 61.0 & 1.1 \\
\hline Gl 825.1 & 6.60 & 0.68 & G5 V & 31.2 & 213 & 0.074 & 0.022 & 25 & 15 & 0.13 & 0.29 & 66.7 & 77.4 \\
\hline Steph 1876 & 12.11 & 1.52 & M1 & 22.4 & 416 & 0.059 & 0.014 & 32 & 13 & -0.35 & 0.22 & 38.2 & 23.0 \\
\hline $\begin{array}{lllll}21 & 14 & 58 & -09 & 07.4\end{array}$ & 13.33 & & & 22.4 & 416 & 0.059 & 0.014 & 32 & 53 & -0.35 & 0.22 & 38.2 & 23.0 \\
\hline Gl 825.2 & 6.75 & 0.63 & G5 V & 23.8 & 418 & 0.065 & 0.015 & 34 & 14 & -0.46 & 0.20 & 37.9 & 25.7 \\
\hline Wo $9733 \mathrm{~A}$ & 4.50 & 0.19 & A5 V & 29.8 & 317 & 0.419 & 0.040 & 275 & 6 & -0.12 & 0.09 & 321.7 & 340.9 \\
\hline Wo 9733 B & 6.90 & & A7 V & 29.8 & 317 & 0.419 & 0.040 & 275 & 14 & -0.12 & 0.09 & 321.7 & 340.9 \\
\hline Gl 826 & 2.44 & 0.22 & A7 IV-V & 15.0 & 774 & 0.019 & 0.007 & 11 & 20 & -1.00 & 0.42 & 5.8 & 1.6 \\
\hline GJ 1262 & 6.97 & 0.73 & G5 V & 20.4 & 204 & 0.190 & 0.036 & 50 & 8 & -0.36 & 0.16 & 121.4 & 60.7 \\
\hline Gl $831 \mathrm{~A}$ & 12.05 & 1.67 & $\mathrm{dM} 4.5 \mathrm{e}$ & 7.3 & 368 & 0.059 & 0.014 & 31 & 18 & -0.52 & 0.22 & 33.0 & 2.1 \\
\hline
\end{tabular}




\begin{tabular}{|c|c|c|c|c|c|c|c|c|c|c|c|c|c|}
\hline Name & $V$ & $B-V$ & Sp. type & $d(\mathrm{pc})$ & Exp. & $C R$ & $\pm C R$ & $\mathrm{Li}$ & $\Delta$ & $h r$ & $\Delta h r$ & $f_{\mathrm{x}-14}$ & $L_{\mathrm{x} 27}$ \\
\hline Gl $831 \mathrm{~B}$ & 14.90 & & & 7.3 & 368 & 0.059 & 0.014 & 31 & 18 & -0.52 & 0.22 & 33.0 & 2.1 \\
\hline G145-043 & 9.25 & 1.05 & $\mathrm{dK} 8$ & 25.1 & 328 & 0.923 & 0.054 & 897 & 5 & -0.15 & 0.06 & 693.9 & 521.5 \\
\hline LP $397-34$ & 12.66 & & $\mathrm{~m}$ & 12.6 & 344 & 0.143 & 0.023 & 74 & 17 & -0.33 & 0.15 & 93.7 & 17.9 \\
\hline Gl 833 & 7.14 & 0.88 & $\mathrm{~K} 2 \mathrm{~V}$ & 14.7 & 430 & 0.059 & 0.014 & 22 & 18 & -0.66 & 0.17 & 28.3 & 7.4 \\
\hline Gl $834 \mathrm{~A}$ & 10.34 & 1.42 & dMO & 21.4 & 657 & 0.113 & 0.015 & 122 & 10 & -0.36 & 0.12 & 72.3 & 39.5 \\
\hline Gl $834 \mathrm{~B}$ & 12.30 & & & 21.4 & 657 & 0.113 & 0.015 & 122 & 10 & -0.36 & 0.12 & 72.3 & 39.5 \\
\hline Gl 835 & 9.88 & 1.50 & M0 e & 13.1 & 354 & 0.073 & 0.017 & 37 & 20 & -0.39 & 0.21 & 45.8 & 9.5 \\
\hline Gl 836.1 & 6.73 & 0.62 & G4 IV-V & 25.6 & 367 & 0.090 & 0.019 & 34 & 14 & -0.77 & 0.14 & 38.1 & 29.8 \\
\hline Gl 836.4 & 12.80 & 1.48 & M1.5: & 35.0 & 193 & 0.074 & 0.022 & 15 & 2 & 0.04 & 0.32 & 62.7 & 91.7 \\
\hline G093-033 & 12.07 & 1.53 & dM3 & 21.1 & 356 & 0.062 & 0.016 & 28 & 4 & -0.66 & 0.21 & 29.8 & 15.9 \\
\hline Gl 836.6A & 4.78 & 0.48 & $\mathrm{~F} 4 \mathrm{~V}$ & 22.4 & 402 & 0.155 & 0.022 & 111 & 7 & -0.18 & 0.13 & 113.8 & 68.4 \\
\hline Gl 836.6B & 6.09 & & $\mathrm{G} 2 \mathrm{~V}$ & 22.4 & 402 & 0.155 & 0.022 & 111 & 10 & -0.18 & 0.13 & 113.8 & 68.4 \\
\hline Gl 836.7 & 5.94 & 0.59 & G0 V & 18.4 & 196 & 0.635 & 0.060 & 296 & 9 & -0.27 & 0.09 & 436.5 & 176.5 \\
\hline Gl 837 & 2.87 & 0.29 & $\mathrm{~A} 6 \mathrm{~m}$ & 11.8 & 345 & 1.655 & 0.125 & 479 & 4 & -0.30 & 0.07 & 1112.2 & 185.9 \\
\hline GJ 1264 & 9.80 & 1.46 & $\mathrm{M} 2 \mathrm{Ve}$ & 16.1 & 175 & 0.861 & 0.073 & 355 & 7 & -0.17 & 0.08 & 637.8 & 198.5 \\
\hline Gl 838 & 5.58 & 0.60 & $\mathrm{G} 2 \mathrm{~V}$ & 15.6 & 334 & 0.067 & 0.017 & 25 & 8 & -0.48 & 0.21 & 38.4 & 11.2 \\
\hline HD $207496 \mathrm{~A}$ & 8.24 & 1.00 & $\mathrm{~K} 3 / 4 \mathrm{~V}$ & 26.4 & 179 & 0.072 & 0.023 & 23 & 21 & -0.52 & 0.24 & 40.3 & 33.5 \\
\hline HD $207496 \mathrm{~B}$ & 10.50 & & & 26.4 & 179 & 0.072 & 0.023 & 23 & 21 & -0.52 & 0.24 & 40.3 & 33.5 \\
\hline Steph 1950 & 12.11 & 1.53 & $\mathrm{dM} 2$ & 31.8 & 343 & 0.281 & 0.030 & 214 & 12 & -0.19 & 0.11 & 205.4 & 249.2 \\
\hline Gl 838.5 & 5.08 & 0.37 & F1 III & 27.6 & 301 & 0.404 & 0.039 & 287 & 4 & 0.07 & 0.10 & 351.1 & 320.5 \\
\hline Gl 840 & 7.78 & 0.92 & dK0 e & 20.3 & 586 & 0.021 & 0.008 & 11 & 19 & -0.40 & 0.36 & 12.9 & 6.4 \\
\hline G018-008 & 11.01 & 1.50 & M1 & 19.8 & 327 & 0.035 & 0.013 & 8 & 8 & -0.77 & 0.35 & 14.7 & 6.9 \\
\hline G188-038 & 12.01 & 1.63 & $\mathrm{~m}+$ & 9.0 & 543 & 0.399 & 0.029 & 513 & 9 & -0.22 & 0.07 & 284.8 & 27.4 \\
\hline Gl 844 & 10.62 & 1.58 & M2 V & 16.4 & 320 & 0.036 & 0.012 & 15 & 33 & -1.00 & 9.99 & 10.7 & 3.5 \\
\hline Gl 845 & 4.69 & 1.06 & $\mathrm{~K} 5 \mathrm{Ve}$ & 3.6 & 414 & 0.260 & 0.029 & 169 & 8 & -0.86 & 0.05 & 97.7 & 1.5 \\
\hline G263-011 & 15.87 & 1.80 & $\mathrm{~m}$ & & 1086 & 0.010 & 0.004 & 11 & 3 & -0.46 & 0.28 & 6.1 & \\
\hline Gl 848 & 3.76 & 0.44 & F5 V & 11.8 & 505 & 0.260 & 0.024 & 261 & 6 & -0.58 & 0.07 & 136.1 & 22.5 \\
\hline HD 210460 & 6.18 & 0.69 & G0 V & 55.6 & 402 & 0.097 & 0.017 & 71 & 8 & 0.28 & 0.18 & 94.9 & 350.5 \\
\hline Gl 850 & 7.24 & 0.79 & K0 & 22.4 & 554 & 0.075 & 0.013 & 69 & 5 & -0.26 & 0.18 & 52.1 & 31.3 \\
\hline G188-047 & 13.62 & & $\mathrm{~m}$ & 19.8 & 498 & 0.075 & 0.014 & 50 & 14 & -0.60 & 0.16 & 38.7 & 18.1 \\
\hline G264-022 & 12.90 & & $\mathrm{~m}$ & 16.1 & 627 & 0.015 & 0.006 & 9 & 24 & -0.02 & 0.38 & 12.1 & 3.8 \\
\hline HD 211472 & 7.51 & 0.81 & $\mathrm{~K} 1 \mathrm{~V}$ & 21.5 & 671 & 0.115 & 0.014 & 118 & 7 & -0.42 & 0.11 & 69.7 & 38.4 \\
\hline G232-062 & 14.00 & & $\mathrm{~m}$ & 21.5 & 671 & 0.115 & 0.014 & 118 & 76 & -0.42 & 0.11 & 69.7 & 38.4 \\
\hline Gl $852 \mathrm{~A}$ & 13.40 & 1.70 & $\mathrm{dM} 4.5 \mathrm{e}$ & 10.0 & 254 & 0.232 & 0.033 & 148 & 16 & -0.04 & 0.14 & 188.3 & 22.7 \\
\hline Gl $852 \mathrm{~B}$ & 14.40 & 1.90 & dM5 e & 10.0 & 254 & 0.232 & 0.033 & 148 & 16 & -0.04 & 0.14 & 188.3 & 22.7 \\
\hline Gl $853 \mathrm{~A}$ & 5.39 & 0.60 & G1 V & 13.6 & 359 & 0.040 & 0.013 & 12 & 15 & -1.00 & 0.15 & 12.1 & 2.7 \\
\hline Gl 853 B & 9.90 & & & 13.6 & 359 & 0.040 & 0.013 & 12 & 15 & -1.00 & 0.15 & 12.1 & 2.7 \\
\hline Gl 854 & 9.23 & 1.15 & dK6 & 24.0 & 561 & 0.017 & 0.007 & 8 & 23 & -0.73 & 0.42 & 7.6 & 5.2 \\
\hline Wo 9779 & 3.84 & -0.05 & $\mathrm{~A} 0 \mathrm{~V}$ & 48.3 & 244 & 0.055 & 0.017 & 22 & 20 & -0.44 & 0.25 & 32.9 & 91.8 \\
\hline LHS 3799 & 13.25 & 1.84 & M4 & 7.4 & 271 & 0.158 & 0.026 & 59 & 13 & -0.06 & 0.17 & 126.1 & 8.4 \\
\hline Gl $856 \mathrm{~A}$ & 11.41 & 1.57 & dM0 e & 16.1 & 552 & 1.263 & 0.049 & 999 & 5 & -0.20 & 0.04 & 915.7 & 283.2 \\
\hline Gl $856 \mathrm{~B}$ & 11.60 & & & 16.1 & 552 & 1.263 & 0.049 & 999 & 5 & -0.20 & 0.04 & 915.7 & 283.2 \\
\hline Gl $857.1 \mathrm{~A}$ & 8.86 & 1.19 & $\mathrm{dK} 7 \mathrm{e}$ & 21.4 & 320 & 0.032 & 0.012 & 16 & 26 & & & 26.7 & 14.6 \\
\hline Gl $857.1 \mathrm{~B}$ & 12.40 & & & 21.4 & 320 & 0.032 & 0.012 & 16 & 26 & & & 26.7 & 14.6 \\
\hline Gl 859 A & 6.21 & 0.62 & G3 V & 20.1 & 274 & 0.847 & 0.058 & 603 & 3 & -0.33 & 0.07 & 555.9 & 268.2 \\
\hline Gl $859 \mathrm{~B}$ & 6.40 & 0.61 & G3 V & 20.1 & 274 & 0.847 & 0.058 & 603 & 14 & -0.33 & 0.07 & 555.9 & 268.2 \\
\hline Gl $860 \mathrm{~A}$ & 9.85 & 1.62 & $\mathrm{M} 2 \mathrm{~V}$ & 4.0 & 427 & 0.449 & 0.034 & 468 & 7 & -0.50 & 0.07 & 253.9 & 4.9 \\
\hline Gl $860 \mathrm{~B}$ & 11.30 & 1.80 & M6 V & 4.0 & 427 & 0.449 & 0.034 & 468 & 7 & -0.50 & 0.07 & 253.9 & 4.9 \\
\hline Gl 862.1 & 6.14 & 0.56 & F7 V & 25.5 & 193 & 0.031 & 0.015 & 7 & 39 & -1.00 & 1.56 & 9.2 & 7.2 \\
\hline Steph 2018 & 12.41 & 1.57 & M3 & 16.4 & 214 & 0.332 & 0.041 & 161 & 28 & -0.19 & 0.12 & 242.2 & 77.9 \\
\hline Gl 863.2 & 5.20 & 0.44 & F3 V & 22.7 & 265 & 0.271 & 0.036 & 143 & 6 & -0.28 & 0.12 & 185.3 & 114.5 \\
\hline Gl 863.3 & 7.58 & 0.66 & G5 V & 32.3 & 411 & 0.021 & 0.010 & 7 & 13 & 0.36 & 0.67 & 21.4 & 26.6 \\
\hline Gl 865 & 11.48 & 1.61 & $\mathrm{k}-\mathrm{m}$ & 14.8 & 462 & 0.424 & 0.033 & 389 & 4 & -0.29 & 0.07 & 286.9 & 75.6 \\
\hline Wo 9791 & 14.00 & & & & 462 & 0.424 & 0.033 & 389 & 28 & -0.29 & 0.07 & 286.9 & \\
\hline G189-030 & 9.41 & & M2 & 18.9 & 608 & 0.017 & 0.006 & 12 & 8 & 0.39 & 0.37 & 18.0 & 7.7 \\
\hline LTT 9123 & 10.92 & & $\mathrm{~m}$ & 12.9 & 277 & 0.031 & 0.013 & 9 & 28 & -1.00 & 2.66 & 9.4 & 1.9 \\
\hline LTT 9124 & 12.64 & & $\mathrm{~m}$ & 12.9 & 277 & 0.031 & 0.013 & 9 & 19 & -1.00 & 2.66 & 9.4 & 1.9 \\
\hline Gl $866 \mathrm{AB}$ & 12.66 & 1.98 & M5 e & 3.5 & 161 & 0.197 & 0.039 & 57 & 18 & -0.50 & 0.17 & 111.4 & 1.6 \\
\hline Gl $867 \mathrm{~A}$ & 9.10 & 1.51 & $\mathrm{dM} 2 \mathrm{e}$ & 8.6 & 225 & 3.803 & 0.187 & 999 & 10 & -0.24 & 0.05 & 2676.6 & 239.2 \\
\hline Gl $867 \mathrm{~B}$ & 11.45 & 1.60 & $\mathrm{dM} 4 \mathrm{e}$ & 8.6 & 225 & 3.803 & 0.187 & 999 & 10 & -0.24 & 0.05 & 2676.6 & 239.2 \\
\hline Gl $867.1 \mathrm{~A}$ & 8.51 & 0.78 & G8/K0 V & 39.4 & 143 & 0.102 & 0.030 & 32 & 19 & -0.08 & 0.31 & 80.8 & 149.9 \\
\hline Gl $867.1 \mathrm{~B}$ & 8.60 & & dG9 e & 39.4 & 143 & 0.102 & 0.030 & 32 & 19 & -0.08 & 0.31 & 80.8 & 149.9 \\
\hline Gl 868 & 7.82 & 1.13 & $\mathrm{~K} 5 \mathrm{Ve}$ & 13.6 & 276 & 0.042 & 0.015 & 12 & 28 & -0.64 & 0.22 & 20.6 & 4.5 \\
\hline Wo 9793 & 10.75 & 1.42 & M0 & 35.7 & 108 & 0.070 & 0.028 & 16 & 25 & -0.49 & 0.65 & 40.2 & 61.3 \\
\hline Gl 873 & 10.26 & 1.61 & $\mathrm{dM} 4.5 \mathrm{e}$ & 5.0 & 560 & 5.384 & 0.098 & 999 & 7 & -0.16 & 0.02 & 4017.5 & 122.5 \\
\hline Gl 875.1 & 11.63 & 1.55 & $\mathrm{dM} 3.5 \mathrm{e}$ & 14.2 & 525 & 0.469 & 0.031 & 550 & 8 & -0.17 & 0.07 & 347.4 & 84.1 \\
\hline Gl 879 & 6.48 & 1.10 & $\mathrm{~K} 5 \mathrm{Ve}$ & 7.6 & 150 & 0.526 & 0.065 & 185 & 13 & -0.48 & 0.10 & 303.5 & 21.2 \\
\hline Gl 880 & 8.67 & 1.50 & $\mathrm{dM} 2 \mathrm{e}$ & 6.9 & 419 & 0.036 & 0.011 & 20 & 17 & -0.34 & 0.28 & 23.4 & 1.3 \\
\hline Gl 886.2 & 5.11 & 0.29 & F0 IV & 28.6 & 146 & 0.379 & 0.056 & 109 & 6 & -0.03 & 0.15 & 309.0 & 301.8 \\
\hline GJ 1278 & 9.89 & 1.40 & $\mathrm{dM} 1$ & 20.0 & 646 & 0.026 & 0.008 & 18 & 11 & 0.12 & 0.31 & 23.3 & 11.2 \\
\hline Gl 887 & 7.34 & 1.49 & M2 Ve & 3.3 & 148 & 0.137 & 0.037 & 28 & 2 & -0.78 & 0.15 & 57.4 & 0.7 \\
\hline Wo 9809 & 10.82 & 1.42 & dM0 & 24.9 & 598 & 0.549 & 0.032 & 925 & 29 & -0.31 & 0.05 & 365.8 & 272.1 \\
\hline Gl 890 & 10.88 & 1.42 & $\mathrm{dM} 2.5 \mathrm{e}$ & 21.8 & 138 & 0.427 & 0.059 & 108 & 14 & -0.26 & 0.14 & 295.7 & 168.7 \\
\hline GJ 1279 & 8.39 & 1.20 & K5 V & 15.1 & 476 & 0.051 & 0.013 & 26 & 8 & -0.47 & 0.20 & 29.6 & 8.0 \\
\hline HD 218738 & 7.91 & 0.90 & G5 & 29.3 & 408 & 1.928 & 0.070 & 999 & 7 & -0.12 & 0.04 & 1479.5 & 1522.3 \\
\hline Gl 892 & 5.56 & 1.01 & $\mathrm{~K} 3 \mathrm{~V}$ & 6.5 & 497 & 0.023 & 0.009 & 10 & 32 & -0.96 & 0.18 & 7.4 & 0.4 \\
\hline GJ 1282 & 5.66 & 0.51 & F7 V & 20.6 & 374 & 0.635 & 0.043 & 560 & 7 & -0.01 & 0.07 & 524.7 & 265.8 \\
\hline HD 219509 & 8.73 & 1.05 & $\mathrm{~K} 5 \mathrm{~V}$ & 27.2 & 449 & 0.030 & 0.010 & 11 & 60 & -0.54 & 0.28 & 16.6 & 14.8 \\
\hline HD 219495 & 9.04 & 1.11 & K5 V & 27.1 & 449 & 0.030 & 0.010 & 11 & 12 & -0.54 & 0.28 & 16.6 & 14.6 \\
\hline Wo 9818 & 3.99 & 0.40 & F1 III & 22.0 & 339 & 0.097 & 0.020 & 42 & 4 & -0.72 & 0.13 & 43.7 & 25.4 \\
\hline
\end{tabular}




\begin{tabular}{|c|c|c|c|c|c|c|c|c|c|c|c|c|c|}
\hline Name & $V$ & $B-V$ & Sp. type & $d(\mathrm{pc})$ & Exp. & $C R$ & $\pm C R$ & $\mathrm{Li}$ & $\Delta$ & $h r$ & $\Delta h r$ & $f_{\mathrm{x}-14}$ & $L_{\mathrm{x} 27}$ \\
\hline G067-053 & 12.10 & 1.59 & M4 & & 418 & 0.323 & 0.030 & 303 & 13 & -0.40 & 0.08 & 199.8 & \\
\hline $\mathrm{BD}+085036$ & 9.72 & 1.13 & $\mathrm{dK} 8$ & 37.0 & 419 & 0.032 & 0.011 & 15 & 22 & -0.24 & 0.35 & 22.5 & 36.8 \\
\hline HD 219693 & 5.53 & 0.44 & F5 V & 34.6 & 145 & 0.063 & 0.025 & 12 & 8 & 0.44 & 0.39 & 66.7 & 95.6 \\
\hline GJ 1283 & 8.97 & 1.15 & $\mathrm{~K} 4 \mathrm{~V}$ & 19.2 & 313 & 0.035 & 0.014 & 8 & 26 & -0.63 & 0.27 & 17.3 & 7.7 \\
\hline Gl $894.2 \mathrm{~A}$ & 5.20 & 0.79 & G5 IV & 20.7 & 304 & 0.036 & 0.013 & 13 & 20 & -0.79 & 0.32 & 14.8 & 7.6 \\
\hline Gl 894.2B & 7.61 & 0.91 & $\mathrm{~K} 2 \mathrm{~V}$ & 20.7 & 304 & 0.036 & 0.013 & 13 & 21 & -0.79 & 0.32 & 14.8 & 7.6 \\
\hline Gl 894.4 & 7.36 & 0.80 & $\mathrm{~K} 1 \mathrm{~V}$ & 21.9 & 432 & 0.148 & 0.020 & 102 & 10 & -0.49 & 0.12 & 84.5 & 48.6 \\
\hline Gl 895 & 10.04 & 1.50 & $\mathrm{dM} 2 \mathrm{e}$ & 13.1 & 514 & 0.015 & 0.007 & 8 & 8 & -0.58 & 0.34 & 7.9 & 1.6 \\
\hline LHS 543a & 14.59 & 1.66 & $\mathrm{~m}$ & 24.8 & 405 & 0.054 & 0.013 & 32 & 27 & 0.14 & 0.26 & 48.6 & 35.6 \\
\hline G190-028 & 11.87 & 1.52 & M2 & 14.9 & 263 & 0.812 & 0.057 & 612 & 28 & -0.34 & 0.07 & 528.8 & 140.1 \\
\hline G190-027 & 12.44 & 1.61 & M3 : & 14.7 & 263 & 0.812 & 0.057 & 612 & 16 & -0.34 & 0.07 & 528.8 & 136.8 \\
\hline GJ 1284 & 11.16 & 1.51 & $\mathrm{M} 2 \mathrm{Ve}$ & 16.1 & 299 & 0.500 & 0.043 & 426 & 2 & -0.20 & 0.09 & 362.6 & 112.8 \\
\hline HD 221239 & 8.31 & 0.93 & K0 & 25.6 & 764 & 0.017 & 0.006 & 11 & 27 & -0.76 & 0.25 & 7.3 & 5.8 \\
\hline Gl 896 A & 10.38 & 1.54 & $\mathrm{dM} 4 \mathrm{e}$ & 6.2 & 17 & 0.993 & 0.250 & 37 & 80 & 0.05 & 0.25 & 851.3 & 39.7 \\
\hline Gl 896 B & 12.40 & 1.65 & dM6 e & 6.2 & 17 & 0.993 & 0.250 & 37 & 80 & 0.05 & 0.25 & 851.3 & 39.7 \\
\hline Gl 897 A & 10.95 & 1.51 & M3.5 & 15.4 & 315 & 0.888 & 0.055 & 800 & 6 & -0.28 & 0.06 & 606.0 & 172.7 \\
\hline Gl 897 B & 11.40 & & & 15.4 & 315 & 0.888 & 0.055 & 800 & 6 & -0.28 & 0.06 & 606.0 & 172.7 \\
\hline Gl 898 & 8.60 & 1.28 & K5/M0 V & 13.9 & 313 & 0.104 & 0.021 & 48 & 8 & -0.57 & 0.15 & 55.1 & 12.8 \\
\hline Gl 900 & 9.56 & 1.35 & $\mathrm{dM} 0.5$ & 19.3 & 374 & 0.320 & 0.031 & 278 & 1 & -0.13 & 0.09 & 243.9 & 108.8 \\
\hline HD 221851 & 7.91 & 0.84 & K0 & 23.5 & 596 & 0.030 & 0.009 & 18 & 10 & -0.48 & 0.23 & 17.4 & 11.5 \\
\hline CP-49 11759 & 10.09 & 1.37 & M0 & 23.8 & 146 & 0.531 & 0.061 & 182 & 37 & -0.20 & 0.12 & 384.8 & 259.8 \\
\hline BPM 45048 & 12.37 & 1.44 & & 23.8 & 146 & 0.531 & 0.061 & 182 & 37 & -0.20 & 0.12 & 384.8 & 259.8 \\
\hline Wo 9832 & 3.82 & 1.01 & G8 III-IV & 25.8 & 364 & 9.822 & 0.164 & 999 & 5 & -0.07 & 0.02 & 7797.6 & 6229.1 \\
\hline HD 222143 & 6.58 & 0.66 & G5 & 23.1 & 344 & 0.286 & 0.030 & 239 & 5 & -0.36 & 0.10 & 183.2 & 116.9 \\
\hline CD-42 16413 & 11.93 & 1.46 & M3 & 18.5 & 78 & 0.060 & 0.030 & 8 & 43 & -1.00 & 1.58 & 18.0 & 7.3 \\
\hline G068-034 & 14.24 & & $\mathrm{~m}$ & 23.3 & 338 & 0.035 & 0.012 & 10 & 15 & -0.87 & 0.31 & 12.9 & 8.3 \\
\hline LP $463-28$ & 17.90 & & $\mathrm{~g}$ & 23.3 & 338 & 0.035 & 0.012 & 10 & 9 & -0.87 & 0.31 & 12.9 & 8.3 \\
\hline Gl 902 & 7.07 & 0.99 & K3 V & 11.4 & 462 & 0.029 & 0.010 & 12 & 11 & -1.00 & 0.44 & 8.8 & 1.4 \\
\hline Gl 903 & 3.21 & 1.03 & K1 IVe & 13.8 & 977 & 0.014 & 0.005 & 12 & 8 & -0.78 & 0.27 & 5.9 & 1.3 \\
\hline Gl 904 & 4.13 & 0.51 & F7 V & 13.8 & 373 & 0.111 & 0.019 & 73 & 5 & -0.89 & 0.07 & 40.1 & 9.1 \\
\hline Gl 905 & 12.29 & 1.90 & dM6 e & 3.2 & 350 & 0.177 & 0.024 & 100 & 5 & 0.15 & 0.14 & 161.3 & 1.9 \\
\hline Wo $9836 \mathrm{~A}$ & 4.48 & -0.04 & B9 V & 47.2 & 272 & 0.583 & 0.048 & 377 & 10 & -0.12 & 0.08 & 447.5 & 1191.2 \\
\hline Wo 9836 B & 11.00 & & & 47.2 & 272 & 0.583 & 0.048 & 377 & 10 & -0.12 & 0.08 & 447.5 & 1191.2 \\
\hline GJ 1289 & 12.57 & 1.60 & $\mathrm{k}$ & 8.1 & 453 & 0.105 & 0.018 & 79 & 15 & -0.45 & 0.14 & 62.2 & 4.9 \\
\hline G241-068 & 11.30 & 1.40 & M1.5 & 28.9 & 581 & 0.317 & 0.025 & 400 & 9 & -0.30 & 0.07 & 212.8 & 212.7 \\
\hline GJ 1290 & 13.30 & 1.59 & $\mathrm{~m}$ & 22.0 & 322 & 0.112 & 0.020 & 83 & 3 & -0.11 & 0.17 & 86.5 & 50.2 \\
\hline G273-130 & 14.50 & & $\mathrm{~m}$ & 9.0 & 126 & 0.078 & 0.031 & 9 & 35 & -0.39 & 0.32 & 48.8 & 4.7 \\
\hline Gl 907.1AB & 9.86 & 1.26 & dM0 & 27.0 & 353 & 0.404 & 0.035 & 327 & 11 & -0.24 & 0.09 & 284.5 & 248.7 \\
\hline G275-106 & 12.40 & & M3.5: & 18.5 & 301 & 0.187 & 0.027 & 79 & 7 & -0.32 & 0.14 & 123.5 & 50.7 \\
\hline Gl 908 & 8.98 & 1.48 & M2 Ve & 6.0 & 414 & 0.051 & 0.013 & 27 & 10 & -0.31 & 0.23 & 34.1 & 1.5 \\
\hline G029-069 & 13.57 & & dM4 : & 20.8 & 432 & 0.050 & 0.013 & 21 & 11 & -0.84 & 0.17 & 19.1 & 9.9 \\
\hline Gl 908.1 & 9.34 & 1.26 & $\mathrm{dK} 8$ & 24.9 & 299 & 0.034 & 0.013 & 12 & 55 & -1.00 & 1.04 & 10.3 & 7.6 \\
\hline Gl 909 A & 6.40 & 0.98 & $\mathrm{~K} 3 \mathrm{~V}$ & 10.8 & 747 & 0.419 & 0.025 & 795 & 9 & -0.40 & 0.05 & 259.4 & 36.1 \\
\hline Gl 909 B & 11.70 & & M2 & 10.8 & 747 & 0.419 & 0.025 & 795 & 9 & -0.40 & 0.05 & 259.4 & 36.1 \\
\hline HD 224085 & 7.38 & 1.01 & $\mathrm{~K} 1 \mathrm{~V}$ & 42.4 & 277 & 10.900 & 0.200 & 999 & 1 & -0.15 & 0.02 & 8191.3 & 17596.3 \\
\hline HD 224228 & 8.21 & 0.96 & K3 V & 22.1 & 133 & 0.116 & 0.035 & 20 & 11 & -0.30 & 0.28 & 77.8 & 45.3 \\
\hline G273-186 & 12.93 & & M4 & 16.5 & 331 & 0.256 & 0.030 & 130 & 9 & -0.30 & 0.11 & 172.1 & 56.1 \\
\hline G273-185 & 12.98 & & M4 & 16.5 & 331 & 0.256 & 0.030 & 130 & 13 & -0.30 & 0.11 & 172.1 & 56.1 \\
\hline LP 291-34 & 12.64 & & $\mathrm{~m}$ & 18.0 & 331 & 0.228 & 0.027 & 160 & 7 & 0.05 & 0.12 & 195.4 & 75.9 \\
\hline HD 224635 & 6.47 & 0.55 & $\mathrm{~F} 8 \mathrm{~V}$ & 29.0 & 306 & 0.159 & 0.024 & 87 & 3 & -0.62 & 0.12 & 80.0 & 80.4 \\
\hline Gl $914 \mathrm{~A}$ & 5.81 & 0.67 & G3 V & 12.4 & 369 & 0.029 & 0.011 & 12 & 30 & -0.45 & 0.32 & 17.2 & 3.2 \\
\hline Gl 914 B & 9.00 & & $\mathrm{~K} 6 \mathrm{~V}$ & 12.4 & 369 & 0.029 & 0.011 & 12 & 30 & -0.45 & 0.32 & 17.2 & 3.2 \\
\hline
\end{tabular}

